6.

$$
3 e^{4}=
$$

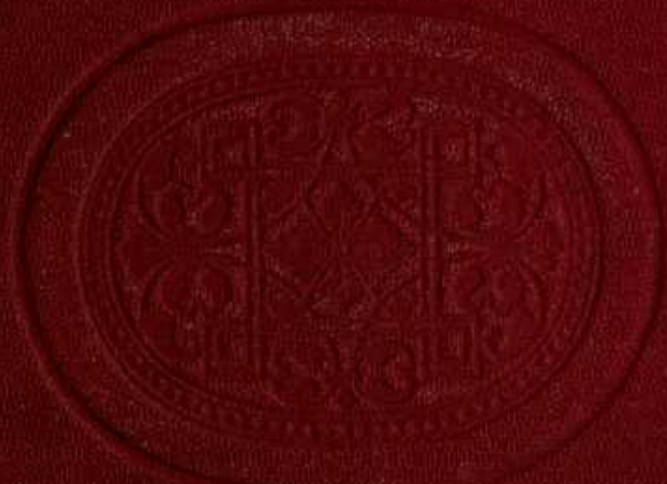




\section{UNIVERSITY OF CALIFORNIA}

AT LOS ANGELES
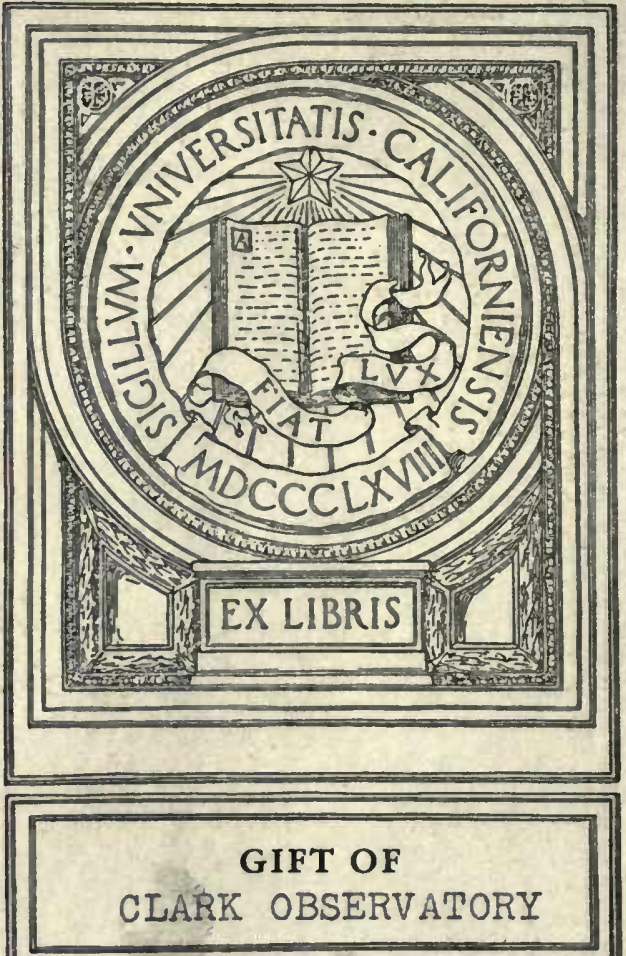



\title{
LIGHT SCIENCE.
}

\author{
Ehird Series.
}


LONDON : PRINTED BY SPOTTISWOOUK AND CO.. NKW-STRRET SQUARF AXD PABLIAEXT STREIT 


\section{LIGHT SCIENCE FOR LEISURE HOURS.}

Ehird Scries:

FAMILIAR ESSAYS ON

SCIENTIFIC SUBJECTS, NATURAL PHENOMENA, \&c.

BY

RICHARD A. PROĆTOR, B.A. САMB.

JONORARY SECRETARY OF THE ROYAL ASTRONOWUCAL SOCIETY; AUTHOR OF 'THE SUN' 'OTHER WORLDS' 'SATURN' 'ESSAYS ON ASTRONOMY'

'THE ORBS AROUND US' ETC.

'Truths of Science waiting to be caught.'-TExxysos.

Cletor Edition.

LONDON :

L ONGMAN S, GREE, AN D CO. 1886. 


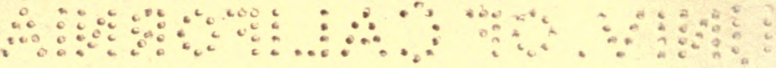

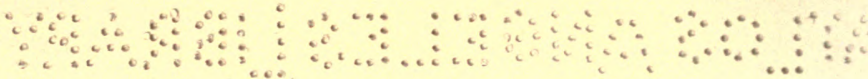




\section{Q 171 \\ Pg4e \\ 1886 \\ ser. 3}

\section{PREFACE.}

IN this volume of Essays I have included a series of papers relating to the great Solar Eclipses which have been successfully observed since the year 1868,- - papers written at the time, and describing the anticipations formed as each eclipse approached, and the results actually obtained during the progress of the eclipse. I have removed portions of those papers which are now practically out of date, but I have left enough to afford what I think will be found an interesting history of the progress of eclipse observation during the past fifteen years.

The other Essays resemble in general character those in the two former series of 'Light Science for Leisure Hours.'

Richd. A. Proctor.

LoNDON : April 1883. 



\section{CONTENTS.}

Great Solar Echipses

PAOK

Two Years Later

The Eclipse of 1870

Yet a Year Later.

74

The Echipse of 1871

102

THE ECLIPSE OF 1878

131

The Earth in Meteoric Shadow . • . . . . 141

Condition of the Laragr Planets . • . . . . 177

A Great Solar Outburst . . . . . . . . 199

Comets . . . . . . . . . . . . 214

The August Meteors . . . . . . . . . 220

LotTery Schemes . . . . . . . . . 225

Conduct and DUty . . . . . . . . . . 232

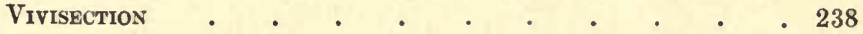

The American Tariff $. \quad . \quad . \quad . \quad . \quad . \quad . \quad .241$

OrIOIN OF OUR RACE. . . . . . . . . . . 252

A late Boat Race . . . . . . . . . . 258

Are We Jews? . . . . . . . . . . . 263

Paradoxes and Paradoxists . . . . . . . . . 269

Influence of Marriage on Death and Crime . . . 281

Increase of the Population . • . . . . . 287

Collisions at Sea during Fog . . . . . . 293

The Eyes of Science • . . . . . . . 297

Great Sun-Spots . . . . . . . . 303 



\section{LIGHT SCIENCE \\ FOR LEISURE HOURS.}

THIRD SERIES.

$+\cdots$

GREAT SOLAR ECLIPSES.

Amovg the total eclipses recorded during historic times, there are some which stand out among the rest on account either of their magnitude or of the historical interest associated with them. I propose to give a brief account of the more remarkable solar eclipses whose records have been preserved. Before doing so, however, it may be well to point out the circumstances on which the magnitude of a solar eclipse depends; and to explain why it is that so few eclipses occur which deserve to be ranked among great total eclipses.

The average apparent dimensions of the sun exceed those of the moon. But both bodies vary in apparent magnitude-the moon more than the sun. Perhaps many of my readers will be surprised to learn that we receive fully one-fourth more light from some full moons than from others, owing to the variation of her apparent magnitude. Accordingly, when she is at her largest and the sun at his smallest, she is able to hide III. 
him wholly from our view, and considerably to overlap. his disc all round.

But there is another circumstance besides proximity to the earth which affects the moon's apparent dimensions. She appears to grow larger as she rises above the horizon. I am not referring, of course, to the appearance which she presents to the naked eye. Judged in this way she seems to grow smaller as she rises above the horizon. But when she is measured by any trustworthy instrument the reverse is found to be the case. The cause of the peculiarity is not far to seek. We see the moon, not from the centre of her orbit (that is, the earth's centre), but from a point on the earth's surface-a point, therefore, which is four thousand miles nearer to the moon's orbit. Accordingly, if the moon were directly overhead (which never happens in our latitudes), her distance from us would be diminished by four thousand miles, and she would look proportionately larger. The sun is not affected in this way, because four thousand miles is a mere nothing in comparison with the enormous distance at which the sun is removed from us. Accordingly, other things being equal, the higher the moon is at the time of a total eclipse, the greater is the eclipse.

In order, therefore, that an eclipse may be as great as possible, the sun should be as far as possible from the earth, which happens about the beginning of July; the moon should be as near as possible to the earth, which happens (roughly speaking) once in every 
lunar month; and the sun and moon should be almost immediately overhead, which can only happen at midday in tropical countries. It will readily be conceived how seldom these conditions can be fulfilled (in combination with the other conditions which determine the occurrence of an eclipse at all). In fact it has never yet happened that any very close approach has been made to the simultaneous fulfilment of all the conditions.

I proceed to discuss a few of the most remarkable eclipses recorded by ancient historians.

It is rather singular that no eclipses are recorded in the Bible. There have been some astronomers who have imagined that the 'going back of the shadow upon the dial of Ahaz' was caused by a partial eclipse of the sun. But this supposition seems too fanciful to be admitted, even if it were the case that a partial eclipse could have caused the retrogression of the shadow. We are told distinctly that the 'going back of the shadow' was a miraculous, not a natural event: and even if this were not so, or if we might infer that it was the prophet's foreknowledge of an approaching eclipse which constituted the miracle, yet it may readily be shown that no partial or total eclipse could produce the effects described. Such an eclipse undoubtedly causes an irregularity in the motion of the shadow on a dial; the shadow at first moves more slowly, afterwards more quickly, than it would otherwise do, but it cannot possibly go back.

The first important eclipse whose records have B 2 
reached us is that which occurred in the year 584 B.c. It took place, Herodotus relates, while the Medians and Lydians were engaged in battle. He thus describes the occurrence:- ' The war had continued between the two nations with balanced success for five years. In the sixth year of the war another battle took place; and after both sides had fought with great advantage, and when the engagement was growing warm, the day was suddenly turned into night. This had been foretold to the Ionians by Thales the Milesian, who predicted the time of the year in which it would happen. The Lydians and Medes, seeing that the day had given place to night, desisted from combat, and were equally anxious to make peace.' Astronomers and historians had for a long time been in doubt respecting the date of this remarkable eclipse. The astronomical difficulty of the question is connected with an interesting peculiarity of lunar motion, into which we need not now enter. Until this peculiarity had been mastered, which has only happened quite recently, Baily's supposition that the eclipse must have occurred in the year 609 B.c. was accepted as the best solution of the difficulty. But Sir G. Airy has now proved beyond a doubt that the eclipse took place on May 28, in the year 584 B.c., the very year assigned to the event by Cicero and Pliny.

Xenophon mentions a remarkable eclipse which led to the capture of Larissa by the Persians. During the retreat which was so ably conducted by Xenophon, the Greeks passed ' a large deserted city called Larissa, formerly inhabited by the Medes. Its walls were 
twenty-five feet thick and 100 feet high; its circumference two parasangs; it was built of burnt brick, on a foundation of stone twenty feet high. When the Persians conquered the Medes, the Persian king besieged this city, but was unable to capture it till a cloud hid the sun wholly from view, when the inhabitants withdrew in great fear, and the city was captured.' Xenophon mentions that the Greeks, after passing Larissa, reached another deserted city called Maspila. Layard has identified Larissa with the modern Nimroud, where there still exist the very ruins described by Xenophon; Mespila he identifies with the modern Mosul. Of course it is impossible to doubt that a total eclipse of the sun, and not the mere concealment of the sun under a cloud, was the cause of the city's capture. Sir G. Airy has shown that this interesting event occurred on May 19, 556 в.c.

Another eclipse has been examined by Sir G. Airy which had given great trouble to historians.' This is the eclipse which took place when Xerxes was advancing with his army from Sardis to Abydos. Herodotus relates that just as the army was setting forth the sun suddenly disappeared from its place in the heavens, though there were no clouds, and the sky was perfectly clear ; ' thus,' says he, 'the day was turned into night.' Sir George Airy, however, refers this description to the total eclipse of the moon, which took place on March 13, 478 в.c. No total eclipse of the sun appears to be reconcilable with the account of Herodotus, and therefore it seems reasonable to infer that there is an error 
of some sort in his narrative. But explaining an eclipse of the sun as an eclipse of the moon is simply preposterous.

It is singular how often the occurrence of a total eclipse is connected with the military and naval undertakings of ancient nations. Most of my readers must remember the narrative of the total eclipse which seriously threatened the success of the expedition of the Athenians under Pericles against the Lacedæmonians. 'The whole fleet was in readiness, and Pericles on board his own galley, when there happened an eclipse of the sun. The sudden darkness was looked upon as an unfavourable omen, and threw the sailors into the greatest consternation. Pericles, observing that the pilot was much astonished and perplexed, took his cloak, and having covered his eyes with it, asked him if he found anything terrible in that, or considered it as a bad presage? Upon his answering in the negative, Pericles said, "Where is the difference, then, between this and the other, except that something bigger than my cloak causes the eclipse?",

But perhaps the most interesting of all the problems with which ancient eclipses have supplied our modern astronomers, is that which is connected with what is termed the eclipse of Agathocles. After his defeat by the Carthaginians, Agathocles was besieged by them in Syracuse. But, taking advantage of a relaxation in the vigilance of the blockading fleet, occasioned by the approach of a fleet which had been sent for his relief, he 
quitted Syracuse, and passing over into Africa, waged for four years a successful war against the Carthaginian forces. It is related by Diodorus Siculus that the voyage to Africa occupied six days, and that on the second day of the journey an eclipse occurred, during which the darkness was so great that stars became visible in all directions. There can be no doubt, therefore, that the eclipse was a total one. But it has been found difficult to reconcile this account with the calculated path of the moon's shadow during the only total eclipse which corresponds with the historical and chronological details of the event. Baily's calculation of the eclipse threw the shadow about 200 miles from the most southerly position which can possibly have been attained by Agathocles on the second day of his journey from Syracuse. The labours of Sir G. Airy, founded on improved tables of the lunar motions, have been more successful; and he has shown that the northern limit of the zone of total shadow must have passed some seventy or eighty miles south of Syracuse-a distance which might readily have been traversed by Agathocles within the time named.

It is related by Philostratus, in his Life of Apollonius, that a singular phenomenon preceded and announced the death of the Emperor Domitian. 'A certain crown, resembling the Iris, surrounded the sun's disc and hid his light.' I cannot doubt that reference is here made to a total eclipse of the sun, and calculation shows that such an eclipse occurred in the year ninety-five of the present era. 
I pass to the records of eclipses which have occurred more recently.

William of Malmesbury relates that the eclipse of August 2, 1133, presaged the death of Henry I. 'The elements showed their grief,' he says, ' at the passing away of this great king. For on that day the sun hid his resplendent face at the sixth hour, in fearful darkness, disturbing men's minds by his eclipse.'

Seven years later another remarkable eclipse occurred which is thus referred to by the same writer :'In Lent, the sun and the moon darkened about noontide, when men were eating; and they lighted candles to eat by. That was the thirteenth day before the calends of April.' (The worthy chronicler might as well have adhered to the more usual method of expressing the date.) 'Men were very much struck. with wonder.' ' The darkness became so great,' he' says elsewhere, "that men feared the ancient chaos was about to return, and on going out, they perceived several stars around the sun.'

Amongst all the eclipses hitherto mentioned there is only one-viz. the eclipse of Thales-which is comparable with that of August 17. And among more recent eclipses there is only one other approaching it in magnitude. This eclipse, which occurred on June 17, 1433, was visible in Scotland, and was long remembered in that country as ' the Black Hour.' It occurred at about three o'clock in the afternoon, and the records preserved respecting it relate that nothing was visible during the height of the totality. Professor 
Grant considers that 'this last remark is a manifest exaggeration.' Be this as it may, there can be no doubt that the eclipse was one of unusual extent, for the mathematician Maclaurin found that ' at the time of its occurrence the sun was only two degrees from apogee, the moon not more than thirteen degrees from perigee.' But neither in this eclipse nor in that of Thales did the totality last so long as during the eclipse of 1868 .

In 1598 another total eclipse occurred which was visible in the British Isles. The day of the eclipse was remembered for a long time afterwards as 'Black Saturday.' In a similar way the day of the total eclipse of 1652 was named 'Mirk Monday' by the people of Scotland, and although the eclipse has long since been forgotten, the expression is still used in many parts of that country.

It is singular that none of the eclipses I have recorded had led to any observations of any value to the physical inquirer. Modern eclipses, on the contrary, derive their chief interest from observations of this sort.

In the total eclipse of 1706 , which was observed at Montpelier, and a variety of other places in Western and Central Europe, the bright stars Aldebaran and Capella, and the planets Venus, Mercury, and Saturn, were visible to the naked eye. 'Bats flew about as they do at dusk. Fowls and pigeons flew hastily to their roosts. Cage-birds were silent, and hid their heads under their wings. Animals at labour in the fields stood still.' Duillier relates that at Geneva 
the Council were compelled to close their deliberations, as they could see neither to read nor write. ' In many places people fell prostrate on the ground, and prayed with earnestness, imagining that the Day of Judgment was come. From the tops of the Swiss mountains as many stars were seen as at the time of full moon. A peculiar colour overspread the sky, resembling neither the darkness of night nor the mixed colours of the twilight sky. Even those who were prepared for the spectacle were appalled by the solemn gloom which fell upon the face of nature.'

Halley speaks in similar terms of the last total eclipse which was visible in London. It took place in the year 1715. 'I forbear,' says Halley, 'to mention the chill and damp which attended the darkness of this eclipse, of which most spectators were sensible and equally judges. Nor shall I trouble you with the concern that appeared in all sorts of animals, birds, beasts, and fishes, upon the extinction of the sun, since ourselves could hardly behold it without some sense of horror.'

The eclipse of May 2, 1733, is remarkable as being the first in which the singular appearances termed the 'red prominences' were observed. 'Four spots of a reddish colour were seen near the limb of the moon, but not in immediate contact with it.' The chief interest attending the observation of total eclipses is at present centred on these mysterious protuberances. It has been shown very clearly that they belong to 
the sun, but what they may be, or what tremendous processes going on within his atmosphere they may be held to indicate, remains as yet unknown. It is hoped that the long duration of the totality of the approaching eclipse, and the circumstance that it will be possible to observe the eclipse at several points along the shadow's track (it will be remembered that this was written in 1868) will enable astronomers to gain some knowledge respecting the red prominonces. Yet more hopeful is the fact that now, for the first time, the subtle analytical power of the most wonderful instrument of research yet invented-the spectroscope-will be applied to examine these strange solar excrescences.

I pass over several total eclipses to come to the first of those which have been made the object of scientific expeditions. The eclipse of July 8, 1842, which was visible in the north of Italy, and in parts of France, Germany, and Russia, aroused an intense interest among European astronomers. The leading observers of France, Italy, England, Germany, and Russia repaired to various suitable stations along the line of central eclipse. M. Arago went to Perpignan, M. Valz to Marseilles, M. Petit to Montpelier; M. Carlini went to Milan, MM. Santini and Conti to Padua; the Astronomer Royal went to Superga, Baily to Pavia; MM. Schumacher and Littrow awaited the eclipse at Vienna; and lastly, the Russian observers, O. Struve and Schidlowski, went to Lipesk. All these observers were fortunate in obtaining excellent views 
of the phenomenon. I shall quote M. Arago's interesting description of the occurrence :-

'At Perpignan, persons who were seriously unwell alone remained within doors. As soon as day began to break, the population covered the terraces and battlements of the town, as well as all the little eminences in the neighbourhood, in hopes of obtaining a view of the sun as he ascended above the horizon. At the citadel we had under our eyes, besides numerous groups of citizens established on the slopes, a body of soldiers about to be reviewed. The hour of the commencement of the eclipse drew nigh. More than twenty thousand persons, with smoked glasses in their hands, were examining the radiant globe projected upon an azure sky. Although armed with our powerful telescopes, we had hardly begun to discern the small notch on the western limb of the sun, when an immense exclamation, formed by the blending together of twenty thousand different voices, announced to us that we had anticipated, by only a few seconds, the observation made with the unaided eye by twenty thousand astronomers equipped for the occasion, whose first essay this was. A lively curiosity, a spirit of emulation, the desire of not being outdone, had the privilege of giving to the natural vision an unusual power of penetration. During the interval that elapsed between this moment and the almost total disappearance of the sun, we remarked nothing worthy of relation in the countenances of so many spectators. But when the sun, reduced to a very narrow filament, began to throw 
upon the horizon only a very feeble light, a sort of uneasiness seized upon all; every person felt a desire to communicate his impressions to those around him. Hence arose a deep murmur, resembling that sent forth by the distant ocean after a tempest. The hum of voices increased in intensity as the solar crescent grew more slender; at length the crescent disappeared, darkness suddenly succeeded light, and an absolute silence marked this phase of the eclipse, with as great precision as did the pendulum of our astronomical ckock. The phenomenon in its magnificence had triumphed over the petulance of youth, over the levity which certain persons assume as a sign of superiority, over the noisy indifference of which soldiers usually make profession. A profound stillness also reigned in the air ; the birds had ceased to sing. After an interval of solemn expectation, which lasted about two minutes, transports of joy, shouts of enthusiastic applause, saluted with the same accord, the same spontaneous feeling, the first reappearance of the rays of the sun. To a condition of melancholy, produced by sentiments of an indefinable nature, there succeeded a lively and intelligible feeling of satisfaction, which no one sought to escape from or moderate the impulses of; to the majority of the public the phenomenon had arrived at its term. The other phases of the eclipse had few attentive spectators, beyond the persons specially devoted to astronomical pursuits.'

M. Arago quotes also a beautiful anecdote in illustration of the peculiar influence produced by the total 
eclipse of the sun's light, and of the joy which springs unbidden to the heart at the return of his beams. A little girl was watching her flock when the sun began to be darkened. As it gradually lost its light she became more and more distressed, and when at length it disappeared altogether her terror was so great that she began to weep and to cry out for help. 'Her tears were still flowing when the sun sent forth his first ray. Reassured by his light, the child signed herself with the cross, exclaiming, in the patois of the province, " O, beou Souleou !" (oh, beau soleil !)'

Remarkable effects were produced on birds and animals by the sudden darkness. Bats and owls came out from their retreats; domestic fowls went to roost; and swallows were seized with so great a terror that in some places they were caught in the streets. A herd of cattle grazing in the fields near Montpellier ' formed themselves into a circle, their heads directed outwards, as if to resist an attack.' Horses and oxen employed in the fields ceased from their labours when the sun was totally eclipsed, and lay down, neither whip nor spur availing to induce them to resume their work until the sun's light returned. On the other hand, M. Arago states that 'the horses employed in the diligences continued to pursue their courses without seeming to be in the slightest degree affected by the phenomenon.' During this eclipse, also, it was noticed that several plants closed their leaves.

The close accordance between the calculations of mathematicians and the observed circumstances of the 
eclipse excited great attention, and led scientific as well as unlearned men to contemplate with admiration the perfection and regularity of the movements of the celestial bodies. 'All the accounts respecting this eclipse,' says Signor Piola, 'contain reflections on the perfection of that great machine of the universe, whose movements are so regular that the astronomer is enabled, long beforehand, to predict their effects with unfailing precision; and from contemplating the machine, it was natural to ascend to the Supreme Antificer. While this idea swells in the mind there is another which at the same time shrinks into insignificance-that suggested by contemplating the position of man in the midst of creation. The magnificence of the scale upon which the phenomena of the eclipse, whether atmospheric or celestial, took place, was patent to every spectator. The extensive coloration of an unusual hue that was visible; the rapid changes which occurred; above all, the obscurity which settled over nature like the funereal pall thrown over a dead body, and whose subsequent withdrawal in an instant operated like a resurrection;-all this produced on the mind a mixture of profound and indefinable impressions which it will be pleasing to hold long in remembrance.'

And in conclusion I would remark how unworthy of the philosopher and student of nature is that spirit which leads men to look with less admiration on natural phenomena that have received their interpretation from the labours of scientific men. No mystery 
of nature has ever yet been unveiled without disclosing what is yet more mysterious. Copernicus revealed the secret of the solar system, to leave undetected the laws which harmonise the planetary motions. It was Kepler's boast that he had revealed these laws, but he left men to admire without understanding their perfection and harmony. Then Newton upraised the veil and disclosed to our admiration the noble law of gravitation which sways all systems through the universe. But we have more now to perplex us, more to reveal to us the insignificance of our powers, more to make us recognise an infinitely wise Creator, than had the simple Chaldæan shepherds, who

Watched from the centres of their sleeping flocks

Those radiant Mereuries, that seemed to move, Carrying through æther, in perpetual round,

Decrees and resolutions of the gods.

If our higher knowledge of the mysteries of nature should lead us to recognise less clearly the author of those mysteries, it would have been better to have never gained that higher knowledge. Our words and works should be worthy of our new light.

Let knowledge grow from more to more,

But more of reverence in us dwell;

That mind and soul according well, May make one music as before, But raster. We are fools and slight;

We mock Thee when we do not fear:

But help thy foolish ones to bear-. Help thy rain worlds to bear thy light.

The next great eclipse is that of July 28, 1851, which was visible in Sweden; the other is the eclipse 
of July 18, 1860, which was visible in Spain, and led to the interesting ' Himalaya expedition.'

The totality lasted nearly twice as long in the eclipse of 1851 as in that of 1842. Sir G. Airy, who had witnessed the earlier eclipse, was one of a distinguished company which left England for Sweden to observe the eclipse of 1851. ' $\mathrm{I}$ have no means of ascertaining,' he writes, 'whether the darkness really was. greater in the eclipse of 1842. I am inclined to think that in the wonderful, and I may say appalling, obscurity, I saw the grey granite hills, within sight of Hvaläs, more distinctly than the darker country surrounding the Superga. But whether because, in 1851, the sky was much less clouded than in 1842 (so that the transition was from a more luminous state of sky to a darkness nearly equal in both cases), or from whatever cause, the suddenness of the darkness in 1851 appeared to be much more striking than in 1842. My friends who were on the upper rock, to which the path was very good, had great difficulty in descending. A candle had been lighted in a lantern about a quarter of an hour before the totality; and M. Hasselgren was unable to read the minutes of the chronometer's face without having the lantern held close to the chronometer.'

During this eclipse the red prominences were seen with remarkable distinctness. Airy at Gottenburg, Hind and Dawes at Rœvelsburg, Lassell at the Trollhätten Falls, and other observers, took drawings of these remarkable appearances; and the agreement III. 
between the drawings is such as to leave no doubt of the care with which those observers examined and recorded what they saw. Round one part of the black limb of the moon there was seen a serrated band of rose-pink light, in another place a pyramidal red mountain, in a third a curved streak of red light formed like a Turkish scimetar, and in a fourth a red detached cloud, which Airy and Lassell picture as nearly circular in form, while Hind and Dawes represent it as triangular. No doubt could exist that these objects belonged to the sun and not to the moon, since the moon was seen to traverse them; insomuch that on the side towards which she was moving their altitude diminished, while on the opposite side they grew larger until the appearance of the sun's disc in this neighbourhood obliterated them through excess of light.

The observers were especially struck by the perfect distinctness with which these remarkable appearances were exhibited. ' $\mathrm{I}$ had heard them described as but faint phenomena,' says Lassell. 'My surprise and astonishment may therefore be well imagined when the view presented itself to my eyes which $\mathrm{I}$ am about to describe. In the middle of the (telescopic) field was the body of the moon, rendered visible enough by the light of the corona attended by the apparent projections. These prominences were of the most brilliant lake colour - a splendid pink quite defined and hard. They appeared to me to be not quiescent: but the moon passing over them, and therefore exhibiting them in different phase, might convey an 
idea of motion. They were evidently to my senses belonging to the sun, and not at all to the moon ; for, especially on the western side of the sun, I observed that the moon passed over them, revealing successive portions of them as it advanced. In conformity with this observation also, I observed only the summit of one on the eastern side, though my friends, observing in adjoining rooms, had seen at least two; the time occupied by me in observing with the naked eye not having allowed me to repair again to the telescope until the moon had covered one and three-fourths of the other.... The first burst of light from the emergent sun was exactly in the place of the chief western flame, which it instantly extinguished.'

When we consider the actual dimensions of these prominences we are enabled to form some conception of the importance of the problem which they present to astronomers and physicists. The scimetar-shaped protuberance was estimated to extend fully one-twelfth part of the sun's diameter from his surface. His diameter is known to be eight hundred and fifty thousand miles, so that the height of this singular object was fully seventy thousand miles, or nearly three times the circumference of our globe. Consider, again: the long sierra extending around nearly a quarter of the sun's circumference. This sierra was about twentyfive thousand miles high. Now many of my readers have doubtless seen the ranges of the Alps as they appear. when seen from some distant point in clear weather, and they know how imposing is the aspect 
of these gigantic land-masses. Yet the highest peaks of the Alps are little more than fifteen thousand feet above the sea-lerel. Imagine, then, the magnificence of glowing masses twenty-five thousand miles above the mean level of the sun's surface.

During the eclipse of 1860 , the red prominences again attracted a great deal of attention among astronomers. It will be remembered that many leading English astronomers, amongst whom the Astronomer Royal again figured, took part in the celebrated Himalaya expedition. MM. Leverrier and Goldschmidt of Paris, the Padre Secchi of Rome, and a host of astronomical celebrities, took part in observing the various phenomena, astronomical, physical, and meteorological, which attended the totality of this important eclipse.

It is interesting, in the first place, to compare Sir George Airy's impressions as to the general effect of the totality with those which he formed during the two former eclipses. It is not often that the same observer-and that observer so skilful and eminenthas the opportunity of contrasting together three total eclipses of the sun. In fact, I doubt very much whether any similar case is on record. Hence, a peculiar value attaches to Sir G. Airy's remarks. 'On the progress of the eclipse,' he says, 'I have nothing to remark, except that I thought the singular darkening of the landscape, whose character is peculiar to an eclipse, to be sadder than usual. The cause of this peculiar character I conceive to be the diminution of light in the higher strata of the air. When the sun is. 
heavily clouded, still the upper atmosphere is brilliantly illuminated, and the diffused light which comes from it is agreeable to the eye. But when the sun is partially eclipsed, the illumination of the atmosphere for many miles round is also diminished, and the eye is oppressed by the absence of the light which usually comes from it. . . . I had a wax-candle lighted in a lantern, as I have had at preceding total eclipses. Correcting the appreciations of my eye by reference to this, I found that the darkness of the approaching totality was much less striking than in the eclipses of 1842 and 1851. In my anxiety to lose nothing at the telescope, I did not see the approach of the dark shadow through the air; but, from what I afterwards saw of its retreat, I am sure it must have been very awful.' "About the middle of the totality I ceased my measures for awhile, in order to view the prospect with the naked eye. The general light appeared to me much greater than in the eclipses of 1842 and 1851 (one cloudy, the other hazy)-perhaps ten times as great; I believe I could have read a chronometer at the distance of twelve inches; nevertheless, it was not easy to walk where the ground was in the least uneven, and much attention to the footing was necessary. The outlines of the mountains were clear, but all distances were totally lost; they were, in fact, in an undivided mass of black to within a small distance of the spectator. Above these, to the height perhaps of six or eight degrees, and especially remarkable on the north side, was a brilliant yellow, or orange, sky, without any trace 
of the lovely blush which I saw in 1851. Higher still the sky was moderately dark, but not so dark as in former eclipses.'

Mr. Airy noted a remarkable circumstance in connection with the red prominences. They were not of the same colour as in 1842 and 1851. The quality of the colour was exactly the same-'full-blush red (or nearly lake)-but it was diluted with white' (an evidence of higher temperature), ' and more diluted at the roots of the prominences close to the moon's limb than in the most elevated points.'

Of course the red prominences do not necessarily reach from the sun's surface, as a mountain from the surface of the earth. Masses suspended in the solar atmosphere would appear as prominences resembling mountains, unless they happened to be of comparatively moderate extent, and were seen in such a position that the space between them and the sun's surface became perceptible. Those serrated ridges, therefore, that we see may belong to the upper surfaces of masses suspended high above the true surface of the sun.

Before proceeding, however, to inquire a little into the probable constitution of these marvellous objects, it will be well to give a brief description of what was seen by Continental observers during the last great eclipse. Leverrier says that the first object which he saw in the telescopic field of view when totality had commenced, was ' an isolated cloud, entirely separated from the moon's limb by a space equal to its own size.' $\mathrm{He}$ adds, that the colour of the cloud was a fine rose, 
tinged with violet, and almost white in some parts through exceeding brilliancy. Near this cloud were two others, one above the other, the upper being the smaller; these were very equally illuminated. Elsewhere he saw two elevated prominences close to each other, and in another part a protuberance resembling a tooth. Returning to the point where he had seen two clouds, he found them unaltered in figure. He now directed his attention to the part of the moon's limb behind which the sun was about to appear. Here he saw a long ridge of reddish purple colour, having a serrated outline.

M. Goldschmidt describes the appearance of one of the rose-coloured prominences in the following terms:- The most imposing, as well as complicated of the prominences, which I will call the chandelier, was grand beyond description. It rose up from the limb, appearing like slender tongues of fire, and of a rose colour, its edges purple and transparent, allowing the interior of the prominence to be seen; in fact, I could see distinctly that the protuberance was hollow. Shortly before the end of the totality I saw escape from the rose-coloured and transparent sheaves of light a slight display in the shape of a fan, which gave to the protuberance a real resemblance to a chandelier. Its base, which at the commencement of the totality was noticed to be very decidedly on the black limb of the moon, became slightly less attached, and the whole took an appearance more ethereal or vapourish.' $\mathbf{M}$. Goldschmidt observed that the small jets of light 
disappeared as soon as the sun's rays became visible, but the prominence itself remained distinctly visible nearly five minutes after the reappearance of the sun. The rest of M. Goldschmidt's account corresponds closely with what is described by other observers. I may remark that his opinion respecting the hollowness of his ' chandelier prominence' seems founded on very insufficient evidence. The transparence of the outer parts of the prominence is a proof rather that the central parts were denser than that the prominence was hollow. But all that M. Goldschmidt says that he observed may be accepted with the fullest confidence, though no other observer has described similar appearances; for there has seldom lived so acute and skilful an observer as this astronomer. He was well known to fame as the discoverer of no less than thirteen asteroids, and numbers of nebulæ and variable stars.

The Padre Secchi, of the Collegio Romano, remarks of one protuberance, that the point was ' rather slender and curved, resembling a flame somewhat agitated.' He remarked that as the moon passed across the solar dise so many luminous points appeared on the following edge of the black disc that he was embarrassed which to choose for observation and measurement. The prominences increased in size as the moon glided forwards, and he 'saw, with surprise, an almost continuous arc of purple light instantaneously formed, composed of small protuberances, in that part of the lunar dise where the reappearance of the sun was expected.' He remarks that his observations have convinced him 
- that the protuberances are connected with the sun, and that it is absurd to assert the contrary.'

It appears to me that very little doubt can exist as to the general character of the red prominences, though we are very far from asserting that their exact constitution can be readily determined.

In the first place, it is tolerably clear that they are not fixed in position. No motion has, indeed, been observed in them during the short time that they have continued visible in total eclipses. But we know that the whole of the sun's surface is in a state of continual agitation. The spots break out, vary in form, expand, contract, expand again, whirl around their nuclei, are suddenly spanned by sharply defined bridges of light, and after many such changes vanish altogether. All this while the region around the spots shows obvious traces of a continual flux and reflux of matter. Then, again, there are the periodic variations in the frequency of spots, and of the faculæ and maculæ which accompany them. And although there are only two bands on the sun's surface (corresponding in position to the temperate zones upon the earth's surface) on which these changes take place, yet we have distinct evidence that the great eleven-year period affects the whole surface of the sun. For at the time when spots are least frequent the sun's disc presents-sometimes for several months-an appearance never observed at any other time. Instead of appearing darker round the edge of the disc, it is seen perfectly uniform in tint over its whole surface. This variability in the appear- 
ance of the sun's surface is inconsistent with the existence of masses of matter, fixed in position (or even permanent in character, but unfixed in position) over extensive solar regions.

We have seen the probability that exists that the red prominences are detached from the sun's surface.

We know, thirdly, that they must exist at an inconceivably high temperature.

Lastly, the spectroscope has proved that the sun's light reaches us after passing through an extensive solar atmosphere, consisting of the vapours of many of our best-known metals. The vapour of iron, for instance, forms a part of the sun's atmosphere-much in the same way as aqueous vapour appears as a constituent of our own air.

It seems to me reasonable to conclude from these considerations that the objects called the red prominences are, in reality, solar clouds; only instead of consisting, as our terrestrial clouds do, of visible aqueous vapour (that is, of minute globules of water), they consist of the visible vapours of the various metals which exist in the solar atmosphere. In other words, they are clouds formed by the condensation of the metallic vapours into liquid globules.

[This was written a few days before evidence was obtained, showing that the prominences are gaseousthat is, even more mobile and tenuous than I had inferred. The reasoning was sound, but proved more than was insisted on.]

Leverrier was led by his observation of the eclipse 
of 1860 to associate the solar spots with the red prominences in a manner closely according with the view we have here put forward. ' Observation proves,' he says, 'that the rose-coloured matter is accumulated occasionally on certain points in quantities more considerable than in others, and as the light of the corresponding part of the sun may possibly be found more or less extinguished, we arrive at a natural explanation of the spots on the sun's surface. These spots will exhibit the most varied forms and appearances, subject to the most rapid changes, in a similar manner to what has been already observed, provided they are produced by clouds. They will change their position on the surface of the sun like clouds on the surface of the earth.'

I trust that the great eclipse which is approaching will not pass without adding largely to our knowledge of solar physics. Everything seems favourable - the regularity of the Indian climate; the long range of inhabited country traversed by the shadow; the careful preparation which has been made for spectroscopic observation, and for taking photographic views of the phenomena presented during the totality. All these circumstances, and the exceptional character of the eclipse itself, combine to afford promise of interesting and important discoveries.

Since the above was written I have heard of several other expeditions which have been sent out to view this important eclipse. The Prussian Government has 
sent an expedition to Aden, almost the nearest point at which the eclipse will be visible as a total one. The French Government sends out M. Janssen, the eminent observer, at the head of a well-appointed expedition. The Pope sends out Father Secchi. Mr. Pogson, the superintendent of the Government Observatory at Madras, will also take part in the work of observing the eclipse. He has been supplied by Mr. Huggins, the eminent spectroscopist, with instruments for analysing the light from the corona and the coloured prominences. Certainly the eclipse will be well watched-unless the weather should unfortunately prove unfavourable. Nor will observers at home be altogether idle. The careful survey of the sun's disc for several days before and after the great eclipse will doubtless be carefully attended to by the eminent students of solar physics who have charge of the Kew Observatory. Thus it will be possible to determine what spots, if any, were on or near the boundary of the disc at the moment of totality; and the suspected association between the spots and the coloured prominences will be put to a satisfactory test.

Cornhill Magazine: August 1868. 


\section{TWO YEARS LATER.}

Two years ago, astronomers were looking anxiously forward to an event which they justly thought likely to prove an epoch in the history of solar research. The great eclipse of August 1868 was not only remarkable on account of the great extent of the black shadow cast upon the earth by the moon, but also as the first total eclipse during which the powers of the most wonderful instrument of research ever invented by man were to be applied to the phenomena visible at such a time. The coloured prominences which had so long perplexed astronomers could hardly fail, it was considered, to reveal their secret under the searching scrutiny of the spectroscope. What the light-gathering powers of the telescope had failed to explain, the lightsifting qualities of the spectroscope might be expected to interpret-and that almost at a glance-precisely as they had resolved "so many other questions of interest.

Every one knows how abundantly these expectations were fulfilled. Not at one station only in India were the observers successful in mastering the secret of the coloured prominences, but, by a wonderful piece of good fortune, every single observer who had made arrangements to direct the spectroscope to the solar prominences succeeded in answering the question which had so long perplexed astronomers. From 
Lieutenant-Colonel Tennant at one Indian station, and from Lieutenant Herschel at another, from Rayet and Janssen, and from the Prussian astronomers who observed the eclipse at Aden, came the same answerthe prominences are masses of glowing vapour. A few bright-coloured lines had in an instant taught the great lesson astronomers had been so long waiting for. Had the coloured prominences been mountains, as some had supposed, the spectroscope would have shown the rainbow-tinted streak which speaks of the solid nature of a source of light. Had they been clouds suspended in the solar atmosphere, there would have been seen the rainbow streak crossed by dark lines, corresponding to that structure. But, because they consist of glowing gas, the rainbow-tinted background was wanting; and only a few bright-coloured lines, corresponding to the particular gases present in these mighty flames, were seen along the spectral range.

Then followed one of those strange coincidences which the history of science has so often presented. Janssen, one of the observers of the eclipse, was struck by the thought that since the light from the prominences is thus gathered up-concentrated, so to speak -into a few bright lines, it might be possible to see those lines even when the sun is not eclipsed. It is easy to see why this might be 'possible. The prominences shine faintly when compared with the solar disc; and so, if we use darkening glasses in observing the latter, we obliterate their light altogether. Nay, even if we absolutely get rid of the direct sunlight, 
yet we cannot see the prominences. Sir George Airy tried the experiment long since. He placed a card, out of which a circular aperture had been cut, in such a way that the image of the sun, formed by a powerful telescope, would have been visible in the place whence the card-circle had been removed-the image just filling that space. Under the actual arrangement, however, the light passed through the aperture, and was received into a black bag, where it was quenched. Now, it might have been supposed that by this ingenious method the image of the prominences would have been rendered visible all round the circular aperture. But the glare from the illuminated air was much more than sufficient to obliterate all traces of them. And so it might seem that no means we could adopt would render the prominences visible. But it occurred to Janssen that since the spectroscope turns the solar light into a long streak-which can be made as dim as we please by increasing its length-while the same instrument turns the prominence-light into a few bright lineswhich are unchangeable in brightness-it might be possible to see these lines after sufficiently reducing the light of the rainbow-tinted solar streak. He tried this the day after the eclipse, and found that it was so: he could distinctly see the prominence-lines even when the sun was shining with full splendour.

Janssen sent news of this discovery to Europe, and on a certain day, nearly two months after the eclipse, the letter announcing the discovery was placed in the 
hands of the President of the Imperial Academy at Paris. Five minutes before, however, the president had read a communication from Mr. Warren De La Rue, announcing that an English observer had lighted independently upon the same discovery.

Let me briefly indicate how this had come about, premising that what we have yet to learn from future eclipses is so intimately associated with the history of what we have already learned, that it would be impossible rightly to present the hopes of astronomers respecting the eclipse of next December without considering the progress of past research.

Mr. Huggins, the eminent spectroscopist, had in 1866 examined the light of a star which blazed out suddenly in the constellation of the Northern Crown. He had found that this star owed its great increase of lustre to an outburst of hydrogen flames; for he could distinctly see the bright lines belonging to the spectrum of glowing hydrogen, superposed on the rainbow-tinted streak crossed by dark lines, forming the ordinary spectrum of a star.

It occurred to Mr. Lockyer that if the spectrum of a glowing gas can thus be recognised in the case of a distant star, we might be able to detect masses of glowing gas on our sun, which is relatively so near to us; so that, if the prominences are of this natureas many astronomers even at that time thought probable-we might be able to see their spectral lines even when the sun is not eclipsed. He directed the attention of the Royal Society to this method of obser- 
vation, and urged them to grant a sum for the construction of a suitable spectroscope. With the usual generosity of the Royal Society, such a sum was placed at Mr. Lockyer's disposal. The problem of making a spectroscope which would adequately lengthen out the solar spectrum was successfully solved by $\mathrm{Mr}$. Browning, the eminent scientific optician ; and, finally, some two months after the eclipse of $1868 \mathrm{Mr}$. Lockyer tried the powers of the instrument thus placed in his possession. As already mentioned, the bright-coloured lines of the prominences were distinctly seen with the new spectroscope; and although Janssen's similar observations had been made nearly two months earlier, no question rests on the independent nature of Mr. Lockyer's observation. Indeed, so successfully had Mr. Browning mastered the optical difficulties of the problem, that no doubt whatever can exist that $\mathrm{Mr}$. Lockyer would have been successful, altogether independently of the information afforded him, in the ac. tual case, by the eclipse observations of August 1868 .

But now let us see the position in which spectroscopists stood. The new mode of observing the prominences presented no special difficulties-at least, what difficulties there were referred to the optician rather than the astronomer. Given a telescope of adequate power, armed with a spectroscope spreading out sufficiently the rainbow-tinted streak which forms the solar spectrum, and it became at once possible for any tolerably well-trained observer to make a series of such researches as, twenty years ago, no man of science 
would probably have believed to be possible. The visibility of a certain set of bright lines would demonstrate not only the existence of a prominence of a particular height at one part of the solar disc, but the nature of the gases of which that prominence was constituted. Nay, so far as the existence of a prominence was concerned, one line alone would suffice for the observer's purpose.

But now new results of extreme importance began to be obtained.

One of the first of these was the confirmation of a theory which had been put forward by Father Secchi several years ago. This eminent observer, making use of a particular mode of viewing the sun, had detected signs of the existence all over the sun's surface of a layer of the same coloured matter which forms the prominences. Combining these indications with the observations he had made during the total eclipse of 1860 , he asserted with great, but not unjustified, confidence his belief in the actual existence of this envelope. 'The observation of eclipses,' he remarks, 'furnishes indisputable evidence that the sun is really surrounded by a layer of this red matter, of which we commonly see no more than the elevated points.'

Now the new mode of research was admirably suited to test the views of Father Secchi. In searching around the solar disc, Mr. Lockyer could only here and there find traces of the existence of prominences; but all round the disc he found short bright lines close to the disc's edge, indicating beyond all question that Father Secchi had been right, and that there really exists all over the bright surface of the sun a gaseous 
envelope, corresponding, though not absolutely identical in structure, with the prominences. Mr. Lockyer called this envelope the chromosphere (for chromatosphere), and it would seem no undeserved compliment to the acuteness of one of the most indefatigable of modern astronomers that this envelope should be known in future by the name of Secchi's sierra. ${ }^{1}$

I have said that this envelope is not actually identical in structure with the prominences. It is, in fact, more complicated. Only certain gaseous elements of the sierra seem capable of rising to the enormous height attained by the prominences. In these great masses the principal element is hydrogen, but in the sierra many elements are commonly recognised-such as sodium, magnesium, barium, iron, \&c. - while under favourable circumstances the bright lines in the sierra are so numerous as to indicate the presence of quite a large proportion of the elements which exist in the sun's substance.

But then other modes of research with the spectroscope came into operation. Mr. Lockyer entered into an alliance with Dr. Frankland, one of the most eminent physicists of the day, and very quickly the wisdom and advantage of this course were manifested. It is one of the most promising characteristics of spectroscopic research, that work done in the laboratory becomes available to tell us of the structure of orbs many millions of miles away from us. Dr. Frankland

1 I write thus with perfect knowledge that other astronomers had yet enrlier suspected the existence of the chromosphere. 
soon obtained results which supplied most important information respecting the solar constitution. It had been shown long since by Plücker and Hittorf, that the bright lines which form the spectrum of hydrogen and other gases vary in appearance according to the circumstances of pressure, temperature, and so on, under which the gases give out their light. Here was at once a powerful means of inquiring into the condition of the gases forming the solar envelope. Dr. Frankland was able to confirm the researches of Plücker and Hittorf, and further-he went far towards proving that pressure is the chief circumstance affecting the appearance of the bright lines of hydrogen and other gases. He showed, too, that with a great diminution of pressure some of the bright lines disappear.

Now, turning to the sun, Mr. Lockyer was able through these researches to form at least a probable opinion respecting the pressure at which the hydrogen forming the solar prominences in reality exists. He found that the bright lines are so narrow as to indicate a singularly low pressure-considering the enormous force with which the sun attracts his atmospheric envelope-a pressure actually less, indeed, than that of our own atmosphere. He could trace also the elevations at which some of the lines of the various: elements forming the solar atmosphere vanish-the others remaining - and so, by comparison with Dr. Frankland's elaborate researches, could tell at least with some probability what, is the actual pressure at different heights in the solar atmosphere. It was, in 
fact, much as though an observer on the sun could see our barometric columns standing at a height of thirty inches at the sea level, and at lesser and lesser heights at greater and greater elevations. Precisely as such an observer, supposing him to be acquainted with the nature of the barometric column, could tell the circumstances of pressure at different heights in our atmosphere, so Mr. Lockyer, knowing the gases which form the chromosphere, and informed by Frankland's researches of the interpretation of the vanishing of bright lines, could tell the variations of pressure at different heights in the solar atmosphere.

I have said, however, that the result was not absolutely certain. It is easy to see why this is. Temperature has an undoubted effect upon the bright lines belonging to the gaseous spectra, and it is obvious that the heat throughout the solar atmosphere must far surpass any which our chemists can artificially produce in their laboratory experiments. So that it must still remain open to some question whether we can reason quite so confidently respecting the condition of things in the sun's neighbourhood, as we might if such peculiar relations did not necessarily exist there.

But at present it seems at least a probable inference that the gases forming the prominences are not subjected to very great pressure. And this brings me to the consideration of the phenomena which will undoubtedly occupy the chief attention of observers during the approaching total eclipse of the sun. When the sun is quite concealed from view by the interposing 
moon, there springs suddenly into view a crown of glory all round the moon's black disc, which has for centuries perplexed astronomers. This appearancethe solar corona, as it is called-had been supposed until quite recently to be a solar atmosphere, other interpretations having one by one been abandoned. But then, the corona extends in appearance to a distance at least half as great as the moon's apparent diameter from the lunar disc. So that if in reality it is due to a solar atmosphere, that atmosphere must be at least half the sun's diameter in altitude-that is, upwards of four hundred thousand miles high. Now our own atmosphere is probably not more than one hundred miles high, so thas the solar atmosphereassuming the view we are considering to be correctwould be no less than four thousand times as high as ours; and on this account alone the pressure at its base would enormously exceed the pressure of the air we breathe. But this is not all. The pressure of our air is due wholly to the earth's attraction, and would be increased or diminished if the earth's attractive force were increased or diminished. Now the sun exerts an attractive force so vastly exceeding that exerted by the earth, that if a man could be placed at the sun's surface-remaining uninjured by all other circumstances-he would be crushed flat by his own weight. We can see, therefore, that the atmosphere of the sun would have its pressure enormously increased through this cause also. Combining the two causes, it is not too much to say that the pressure at the sun's 
surface, under such an atmosphere as we have been supposing, would suffice to liquefy if not to solidify the most subtle gases we are acquainted with.

There cannot be any question therefore that the spectroscopic observation of the sun has sufficed to throw very great doubt indeed upon the theory that the corona is due to a solar atmosphere. Or rather, we may fairly say that that theory has been distinctly shown by Dr. Frankland's laboratory researches to be untenable.

- But then there remains the difficulty of explaining what the corona really is. We know that it cannot be a lunar atmosphere, because a number of very exact observations have shown, beyond all possibility of question, that the moon has no atmosphere of appreciable extent, far less such an atmosphere as would account for the corona. Again, the theory which was put forward by De Lisle in the seventeenth century, that the corona is caused by the diffraction of the sun's rays as they pass by the moon, has been disproved by the inquiries of Sir David Brewster.

There is indeed another theory, which hasstrangely enough-been exhumed quite recently. According to this theory the solar corona is simply a phenomenon belonging to our own atmosphere. The theory was first mentioned-though only to be summarily rejected-by Halley, and touched on somewhat contemptuously by other astronomers. It explains the corona as due to the illumination of the upper regions of the air by the sun's rays. We know that if we hide the sun with a globe or disc of any sort, a strong light 
is seen all round the interposed object. And it might seem that since the moon is but a globe somewhat larger than our experimental one, and somewhat farther off, we ought to see a similar light all round the black disc of the eclipsing moon.

But a little consideration will show the fallacy of this reasoning.

When we hold a globe so as just to hide the sun, we do not throw into shadow those upper regions of air from which the atmospheric glare really comes. But when the moon conceals the sun during total eclipse, she causes an enormous shadow to fall right through the whole depth of the air. This shadow, even at its narrowest, that is, where it reaches the earth, has been in many total eclipses fully one hundred miles wide; and as the part of the air capable of reflecting solar light to an appreciable extent is shown by the twilightarch to be but forty or fifty miles high at the outside, we see that, in the case of such eclipses, the moon's shadow in the air is of a vast drum-shaped figure, at least twice as wide as it is high. It is most obvious, then, that to an eye placed at the centre of the vast base of this drum-shaped shadow, no light can possibly come from the air for a wide range all round the place of the eclipsed sun. Imagine a shadow hiding nearly all England and fifty miles high; then to an eye placed, say at Hereford, the upper surface of the shadow would cover an enormous extent of sky, while the eclipsed sun, at the apparent centre of that surface, would be but as a relatively minute circle. 
It need hardly be said that considerations so obvious have not escaped the attention of astronomers. I have said that Halley rejected the atmospheric glare theory, and that other astronomers have spoken of it with but little respect. In quite recent times, competent astronomers, who have had occasion to examine it, have in like manner rejected it. Dr. Harkness, who witnessed the American eclipse, and was led by the study of the corona-as actually seen-to inquire inta the physical nature of the phenomenon, remarks respecting the theory that 'the moon's shadow, at the point where it enters the earth's atmosphere, usually has a diameter of one hundred miles or more, and if it were possible for an observer placed within that shadow to see the illumination of the atmosphere outside of it, the appearance presented would be that of a halo having an interior diameter much greater than the size of the moon.' Dr. Curtis, also-a skilful mathematicianafter exhibiting precisely the same line of reasoning, remarks that 'it is geometrically impossible for an observer near the centre of the shadow to see any portions of our atmosphere which lie beyond the cone of darkness-which portions alone could, of course, under any circumstances be illuminated - in apparent contiguity with the moon's disc.'

Thus we are brought back to the theory that the corona really is a solar phenomenon, while yet we are precluded from supposing that it is a solar atmosphere. What, then, can it be?

Now astronomers hope for much, and for very 
useful information respecting the corona, during the progress of the eclipse of December 1870; and the question may suggest itself, how far it is wise to discuss the subject of the corona now, when in a few months we may be in so much more advantageous a position for theorising respecting it.

This is a consideration well worth dwelling upon; yet the arguments by which we are to deal with it are sufficiently simple. If we have already exhausted all means of inquiry applicable to what we already know respecting the corona, then our proper course is to wait. It would undoubtedly be absurd in that case to attempt to evolve from the depths of our moral consciousness a theory respecting this wonderful and mysterious phenomenon.

But in truth there is little fear of our thus overriding observation. In these days observation progresses with such amazing rapidity that reasoning is left far in the rear. It is not too much to assert that if all observation were from this moment to cease, the students of science would find abundant employment for a decade of years, at least, to come, in examining and utilising the observations which have been already made.

Let us, then, look round our storehouse, and see if we may not at once, and almost at random, light upon a few observations which may serve to help us in interpreting the wonders of the corona.

A few months ago Captain Noble was looking at the planet Venus when almost directly between us and the 
sun. She was so placed with respect to the sun that, had his globe been eclipsed, she would have been seen near the edge of the coronal light. Of course her unillumined side was turned towards us. It appeared darker than the background on which it was projected. Whence came that light which illumined the background? Or rather, what light was it which the globe of Venus concealed? Something beyond Venus undoubtedly-else how could she conceal it? Obviously, then, there is some light where we see the corona when the sun is eclipsed, and that light comes from a region farther off than Venus is when nearest to us. Here is another evidence to strengthen our conviction that the corona is an extra-terrestrial phenomenon, supposing any faint doubts to remain after the evidence already adduced.

We have gone to Venus, seemingly so little associated with the corona, for evidence respecting that phenomenon. Turn we now to other objects which seem at first sight even less likely to give us any information.

Consider thoughtfully the meteor which flashes across the dark background of the sky at night. We know now quite certainly that every falling star has travelled before reaching our atmosphere along an orbit of enormous dimensions. It has been proved further respecting all the meteors whose real path has been determined, that their orbits are very eccentric, insomuch that though they cross the earth's path-otherwise, of course, we should never see them-they pass 
out to distances exceeding in some cases those at which Uranus and Neptune pursue their wide careers. We may conclude, then, of far the greater number of the meteors the earth encounters, that their paths, having their most distant portions so much farther from the sun than our earth, must have their nearest part-to the sun-much closer to the sun than the earth is. So that if meteors were as large as planets, it would necessarily happen that many meteors belonging to systems which the earth encounters would be seen, at certain seasons, shining close by the sun.

But, as a matter of fact, meteors are individually far too minute to be thus seen; nor could all the meteor systems traversed by the earth become visible by the combined lustre of their components.

It is clear, however, that the meteor systems traversed by the earth can be but a few among the meteor systems actually existing, and having paths carrying their components nearer to the sun than the earth is. Taking at random any such path, the chance that the earth's path would cross it is indefinitely small ; so that clearly an indefinite number of such systems must exist in order that the earth might have a fair chance of encountering a single one. And since she actually encounters hundreds, it will be seen how enormous must be the real number of systems actually existing.

This mode of reasoning, though in strict accordance with recognised and certain principles, may not seem convincing at first sight. But, in reality, it will be found that we quite commonly, and as it were uncon- 
sciously, follow the guidance of the principle in ordinary life. We are out walking, suppose, and a drop of rain falls upon us; now, there is no absolute reason why a single drop falling from the sky should not light upon us; yet so certain are we that the odds against such an event are enormous, that we conclude at once that rain is falling over a wide space all around us. Or again, suppose we meet some day five or six persons dressed in a peculiar costume, and not forming one party-we conclude at once that there is to be some gathering of such persons on the day in question. And so in a thousand instances which will occur to every one.

I conclude, then, with the utmost confidence that for every meteor system encountered by the earth there must be thousands which she does not encounter.

And these multitudinous systems, illuminated as they must be by the sun's rays, might very reasonably be expected to become visible under favourable circumstances-as, for example, when the sun is eclipsed. Nay, knowing that the meteors travel in paths resembling those of comets, and in some cases associated in the most intimate manner with the paths of known comets-we may conclude that large numbers of meteors. pass as close to the sun as some comets have been observed to do, or even nearer, for observed comets form but a small proportion of the total number of such objects. Now, Sir John Herschel has shown that the comet of 1843 passed so close to the sun that it must have been subjected to a hent exceeding three and a 
half times that obtained by means of Parker's great lens, which melted such refractory substances as cornelian, agate, and rock-crystal. Meteors so close as this to the sun would be so intensely heated that their inherent light would be even more brilliant than that which they would be capable of reflecting. Many would even be vaporised as they rushed past the point of their nearest approach to the solar orb. We see then that, quite apart from the information which a solar eclipse affords us, we really have just reason for pronouncing with considerable confidence that something very like what the corona appears to be must exist in the sun's neighbourhood.

Now it is well worth noticing that if we suppose the corona really to be caused by the illuminated meteoric systems, we get rid at once of that difficulty which spectroscopic analysis opposed to the theory that the corona is a solar atmosphere. These swiftly rushing meteors would no more tend to increase the pressure at the base of the solar atmosphere than the moon, circling as she does round the earth, tends to increase our own atmospheric pressure.

It happens, too, that such evidence as has hitherto been given by the spectroscope respecting the actual constitution of the corona corresponds very satisfactorily with the conclusions above deduced. I do not enter here into a very particular account of that evidencefirst, because the observations made by different astronomers have not yet been brought into complete accordance; and secondly, because it is confidently 
hoped that the approaching eclipse observations will make abundantly clear that which is at present somewhat confused. But the very diversity of results corresponds with the diverse character of the light which comes-according to the above results-from the meteoric systems near the sun. Meteors simply reflecting solar light would exhibit the rainbow-tinted streak crossed by multitudinous dark lines, which forms the solar spectrum; meteors incandescent through intensity of heat would exhibit a rainbow-tinted spéctrum without dark lines; and lastly, meteors vaporised by heat would exhibit a spectrum of bright lines. The combination of such spectra in varying proportions would quite satisfactorily account for the results hitherto observed by spectroscopists. It is, however, worth noticing that electrical discharges exerted by the sun's action, and taking place between the meteors, would even more completely account for observed results; and perhaps it is only because electricity has come to be regarded as a sort of refuge for the scientific destitute, that men of science have been hitherto unwilling to resort to such an explanation of what has been observed.

But astronomers hope that during the eclipse of next December the spectroscope will be applied much, more effectually than has yet been done to the scrutiny of the solar corona. Photography, too, it is hoped, will be so applied as to exhibit the corona, and not merely, as hitherto, the pink prominences and the more brilliant part of the glare around the sun. Then 
the polariscope is to be applied, though for my own part I have very little faith in the possibility that this instrument can give intelligible and reliable results respecting such a phenomenon as the corona would appear to be. If its light really is of the mixed nature I have described, it can scarcely be but that the polariscopic teachings will be discordant and practically unmeaning.

Last, but not least, a large array of observers have devoted themselves to the scrutiny of the general features of the eclipse. I wish very strongly to indicate the opinion that much remains to be done in this way. It seems to me that in all the eclipses hitherto observed, attention has been somewhat too exclusively directed to the eclipsed sun and its immediate neighbourhood. I write this in the full knowledge of the meteorological and other observations which have been made during eclipses. The class of observation which, as it seems to me, has bèen insufficiently attended to, includes the special study of the varying illumination of the sky, not near the sun, but at all orders of distance from him. Remembering that we know the actual figure, dimensions, and position, from second to second, of that vast shadowcone which the moon projects upon our atmosphere, we can interpret in a very satisfactory manner the apparent changes of illumination in different regions of the sky. From such observations, properly made and studied, more could be learned, I do not hesitate to say, respecting the height of the air, the quality of its 
upper and unattainable regions, and other like subjects, than from all the methods by which as yet men of science have attempted to master these stubborn problems.

It must not be forgotten, however, that the eclipse will last-as far as totality is concerned-for a very brief time. For about two minutes-the exact time cannot be known until the observing stations are decided upon-the sun will be totally hidden from view ; and whatever new information is to be obtained respecting the constitution of the corona, and about other subjects which will occupy the attention of observers, must be gleaned in that short interval of time. Those at home must not be surprised or disappointed, therefore, if the results actually obtained should seem at first sight disproportioned to the expense and trouble involved by the expeditions, or to the number of persons who will take part in the work of observation. Science must be content in such cases to expend a large amount of time and trouble where yet the prospect of remuneration will be but small. All the more credit to those who are ready to join in an enterprise so arduous, and presenting so many chances of failure. 
THE ECLIPSE OF 1870.

Astronomers have passed yet another of those stages which mark their progress towards a fuller knowledge of solar physics. That strange peculiarity of the celestial phenomena presented to us inhabitants of earth, by which our satellite is able just to blot out from view the great central luminary of the planetary system, has yet once more served us in good stead. The few brief seconds during which the sun remained concealed on December 22 last have supplied the means of testing those rival theories which had been propounded respecting the solar corona, and, as it seems to me, of arriving at definite conclusions as to the general nature of this interesting object.

In passing, it may be well to notice how important an influence that peculiarity respecting the apparent dimensions of the sun and moon, to which I have just referred, has exercised on the progress of astronomy. We are so accustomed to the near equality of the sun and moon as respects their apparent size, that we are apt to overlook the fact that this apparent equality must be regarded rather in the light of a fortunate accident than as in any way an essential attribute of the orbs which rule the day and the night. In the whole range of the solar system there is no other instance of so remarkable an association. In Mercury and Venus of course no eclipses of any sort can occur, because these planets have no moons. 
But even in Jupiter, notwithstanding the grandeur of his system of satellites, and though total solar eclipses recur at intervals which must be measured by hours rather than by months, as with us, yet such solar eclipses as we see can never take place. For not one of his moons is capable of just hiding the sun's disc and a very narrow border all round while leaving in view beyond that border the coloured prominences, and beyond the prominences the glory of the corona. If we try to conceive the circumstance of an eclipse of the sun by one of Jupiter's nearest moons, we have to imagine a dark disc capable of obliterating a sun more than thirty times larger than that which is actually seen from Jupiter; and even the farthest of Jupiter's moons covers twice as great a space as the sun. It is easily seen that when a total eclipse is just beginning or just ending, under these circumstances, only a small part of the matter outside the sun can be visible, and nothing resembling that complete ring of such matter, visible to ourselves when the moon obliterates from view the nearly equal solar disc. So also in Saturn, whence the sun must appear as a mere dot of bright light, and in Uranus and Neptune, whence he appears yet smaller, there can be no such eclipses as we inhabitants of earth are favoured with. Hence it may not unreasonably be concluded that terrestrial astronomers alone have any knowledge of the coloured solar prominences and of the corona.

It is worth mentioning, also, that, interesting as are the discoveries which have been recently made during 
solar eclipses, there are other discoveries due also to the observation of total eclipses, though in very ancient times, which are as full of interest. It sounds ineredible, but is nevertheless strictly true, that, owing to comparatively rough observations of ancient eclipses, modern astronomers have learned that the moon is gradually drawing nearer to the earth; and further, that the rate of the earth's rotation on her axis is slowly but surely diminishing, insomuch that at some far distant epoch the day will last as long as a lunar month. Nor do the facts that the approach of the moon will in time be changed into recession, and that the lengthening of the day takes place so slowly that millions of centuries must elapse before it is completed, diminish the interest which attaches to these tokens of mutability in relations which had once been regarded as altogether unchangeable.

But let us turn to those discoveries which belong more especially to the now wide department of science called solar physics.

It does not appear that the ancients had any idea that observations made during total eclipses could afford any information as to the condition of the great central luminary of our system. To them the chief interest of solar and lunar eclipses consisted in the evidence they afforded of the exactness of astronomical computations, and the soundness of the general principles on which those computations were based. Nor do we find that any of the observed phenomena of total eclipses attracted the special attention of ancient 
astronomers. They recognised the corona, and they justly regarded it as the cause; of that light which still remains when the sun's globe is wholly concealed from view; but they formed no theories as to the physical significance of this aureole of light.

Indeed, if we are to reach the time when systematic observations have been made upon the sun with the express object of determining the nature of those appendages which come into view during total eclipse, we must pass over not merely the whole of ancient astronomy, but almost the whole of that portion of the history of modern astronomy which refers to epochs preceding the last thirty years or so.

It was when the eclipse of 1842 was approaching, that for the first time astronomers aroused themselves to a sense of the real importance of the phenomena presented during total eclipse. Then, for the first time, astronomers of repute, armed with instruments of adequate power, placed themselves along the track which the moon's black shadow was to pursue, and severally prepared to glean what knowledge they might respecting the physical habitudes of the solar surroundings.

The expeditions made in 1842 were abundantly rewarded. For it was during that great total eclipse that the coloured prominences were first fairly recognised. More than a century before Vassenius had suspected the existence of some red objects near the eclipsed sun. But strangely enough small attention had been paid to his remarks. And accordingly, the 
astonished world of astronomers learned first, in 1842 , that mighty red protuberances of a nature as yet unexplained, but certainly vast beyond all our powers of conception, surround the surface of our great luminary. It needed but a brief study of the pictures made by those who observed the eclipse, to see that in the first place these phenomena were undoubtedly solar, and secondly, that the real magnitude of some of the prominences was enormously greater than that of the earth on which we live. Whether these were mountains heated to incandescence by the solar fires, or fiery clouds suspended in the solar atmosphere, or lastly, flames rising like mighty tongues from the solar surface, few ventured to pronounce. But it was plainly seen that, whatever they might be, they surpassed all hitherto discovered phenomena within the whole range of the solar system in interest and magnificence. The telescope had hitherto shown nothing which could well be compared with these strange solar appendages. The mountains and valleys in the moon, the lands and seas of Mars, the belts of Jupiter and Saturn, and even the mighty ring-system which girdles the last-named orball these, interesting though they doubtless are in themselves, yet sink into utter insignificance compared with solar appendages so vast that, at a moderate estimate, some of them must have a height exceeding the diameter of Jupiter-the giant of the solar system.

The real existence of the coloured prominences was not admitted, however, without further evidence. In all ages of astronomy there have been those who 
dispute to the last the significance of observed facts. Unfortunately, in this instance, as in others, the suggested doubts exercised a mischievous effect. It was urged loudly by a few astronomers-as Faye, Feilitzsch, and others-that the so-called prominences were mere optical illusions, or else were but a species of lunar mirage. Airy, Baily, the younger Struve, and others, had recorded their experience in vain; fresh observations were called for; and accordingly in $185 \%$, and again in 1860, a host of skilful observers devoted their energies to demonstrate what was in truth a demonstrated fact-the reality of the red protuberances.

Yet the important eclipse of 1860 did not pass altogether without profit. Too many, indeed, of the observers who formed the celebrated 'Himalaya expedition,' as well as of those Continental astronomers who visited the path of the moon's shadow across Spain, were led by the unfortunate doubts of Faye and others to make useless observations. But the successful photographing of the coloured prominences by De La Rue and Secchi sufficed to convert what would otherwise have been a gigantic failure into a success well worthy of record. For the first time astronomers possessed pictures of the prominences which were beyond cavil or question. And further, since De La Rue had been stationed in the west of Spain, while Secchi had placed himself close by the eastern shore, it had become possible to form an opinion of the permanence or mobility of these strange objects. So 
far as the comparison made between these two sets of photographs was concerned, it appeared as though the solar prominences were fixed objects; and some went so far as to conclude definitely that they are real solar mountains.

It was not until the great eclipse of August 1868 that the real nature of the coloured prominences was ascertained. This eclipse was distinguished from all that had ever been observed before by the duration of totality. For more than six minutes the disc of the sun was completely hidden from view. It need hardly be added that the shadow on the earth's surface was exceptionally wide; so that near the middle of totality at any station along the central line the observer was in the centre of a nearly circular region of the earth more than 150 miles in diameter, and to which not a ray of direct sunlight penetrated. All the features of the eclipse were thus observed under singularly favourable circumstances.

In the first place, it was possible to obtain more photographs than on any former occasion. LieutenantColonel Tennant, using a fine 9 -inch reflector made by Mr. J. Browning, F.R.A.S., obtained no less than six photographs, coloured pictures of which lie before me as I write. The first shows a glare of light on the left, where the moon had not as yet covered the last fine line of the sun's disc. Yet we see through the glare the figures of the prominences on that sideshowing 'as through a glass darkly'-and amongst them that mighty horn-prominence whose spiral whorls 
attracted the attention of all who witnessed the eclipse. Then in the succeeding pictures we see the moon's disc gradually passing over this wonderful horn and the prominences lying on the same side; while on the opposite side we see a long range of prominences coming as gradually into view. None of these are comparable in height with the mighty spiral on the left, though some of them are amazing objects, and of dimensions so vast that a globe like our earth placed close by them would seem but as the reriest bubble amid the foam of a storm-wave. In the last picture of all, these prominences on the right show their full proportions as the advancing moon is about to bring the disc of the sun into view on that side. But though the moon has passed thus far towards the left, and though, indeed, all other prominences on the left are concealed from view, yet on that side the spiral horn still towers so loftily as to form the most striking feature of the scene.

But interesting as are these pictures, and forming though they do, despite the success of the American astronomers in August 1869, the most remarkable series of photographs ever taken of the eclipsed sun, the chief interest of the eclipse of 1868 depends on another circumstance. This eclipse was the first during which the powers of the spectroscope had been applied to determine the nature of the coloured prominences; and astronomers looked forward to the result with a degree of interest which was fully justified by the discovery actually effected. 
The spectroscope, applied successfully at all the observing stations, resolved, in a manner there was no misinterpreting, the problem which had so long perplexed astronomers. And the strange answer to their questions was this-that the coloured prominences are masses of gas glowing with intensity of heat. Those vast and seemingly stable protuberances, so enormous that ten globes like our earth placed one upon the other on the sun's surface would not reach their summit, are flames of hydrogen, that familiar element which constitutes so large a proportion of our ordinary gas flames. Or, rather, they are not strictly flames of hydrogen, but whorls of the gas heated to an intense degree of brightness. And other vapours are also present in these vast glowing masses, since the spectrum of the prominence-light shows other lines than those which are characteristic of hydrogen.

I need not recount here in full the interesting history of sequent researches into the prominences. Indeed not the least remarkable feature of that history is the circumstance that the study of the prominences has not continued to be associated (as it had been until the autumn of 1868) with the history of eclipses. First Janssen, afterwards (but independently) Lockyer, succeeded in seeing the bright lines of the prominence spectrum when the sun was shining in full splendour. Then the lower regions of prominence-matter, forming what previous observers had denominated the sierrabut named by Lockyer (who was unaware of its prior discovery) the chromosphere-was analysed with the 
spectroscope, and in the same manner. And lastly came the crowning discovery of all-the recognition, by Dr. Huggins, of the fact that the prominences themselves, as distinguished from the lines of their spectra, can be seen when the sun is not eclipsed. By Huggins's method, Lockyer and Zöllner obtained interesting views of the prominences, and witnessed the strange and in some instances rapid changes to which these objects are subjected. But Respighi of Italy.has been even more successful, or, rather, more systematic in his researches. For he has succeeded in obtaining daily records of the condition of the sun's edge, not in one place only but all round. So that we have every reason to anticipate that before long astronomers will be able to watch the changes of the prominences from day to day as systematically as they already watch the progress of the solar spots. If each day there were a total eclipse, instead of but an eclipse or so per year, we could not have such complete and perfect records of the sun's condition as some of those which Professor Respighi has obtained for every fine day during two or three consecutive months. I have one of his monthly pictorial records before me as I write; and it would certainly be vain for the most skilful artist to attempt, during even so long lasting an eclipse as that of August 1868 , to exhibit the prominences in such detail as we find in each of the daily views forming this record.

Astronomers and physicists had thus successfully analysed the coloured prominences, or, to use Mr. Lockyer's striking, if not strictly elegant, expression, 
"these "things" had been "settled.", Little more could be hoped, as respects these objects, from eclipse observations, however skilfully conducted. But so far the corona had baffled their efforts. Enough had been done to afford tolerably sure evidence as to the general nature and position of the solar corona, yet of its actual structure and constitution very little had been certainly learned. Our knowledge respecting it may be compared to that which astronomers possessed respecting the coloured prominences in 1842 . We could be assured that it really is a solar appendage of some sort-although, precisely as Faye and others had expressed doubts respecting the real existence of the coloured prominences in 1842 , so in 1870 there were those (and, strangely enough, Faye was their leader) who questioned the real existence of the corona, or regarded it as a phenomenon of our own atmosphere. Yet in the opinion of all who were competent to judge, this point was justly regarded as determined. But what the actual nature of the corona might bewhether its light was reflected solar light, or came from incandescent solid matter, or, lastly, was due to glowing vapour-remained unknown.

Yet the doubts thus entertained respecting the constitution of the corona were due rather to the seemingly contradictory nature of the evidence which the spectroscope had thus far supplied than to the absolute want of evidence. Briefly to sum up the results which had been obtained before the eclipse of last December:-In 1868, Tennant had found that the 
spectrum of the corona is a continuous rainbow-tinted streak, without either dark lines or bright. Such a spectrum is given by solid and liquid bodies glowing with intensity of heat. And the inference, therefore, was that the corona consists of minute bodies travelling close by the sun, and owing the greater part of their light to the great heat with which they are transfused. But the American observers in 1869 , or at least some of them, found that besides the ribbon of rainbow-tinted light, the spectrum of the corona shows bright lines. Some observers saw only one bright line, others saw three. This observation would indicate that a portion of the coronal light comes from a gaseous source; and from the position of one of the bright lines, Professol Harkness was led to the strange conclusion that the glowing vapour of iron is a constituent of the solar corona! Yet further, because the position of these coronal lines corresponded with the pasition of the bright lines seen in the spectrum of the aurora, Professor Young, one of the most skilful of the American spectroscopists, came to the conclusion that the corona is a perpetual solar aurora!

The observations of the American astronomers and physicists were not accepted by all. No valid reasons were given, indeed, for rejecting them, but they were pronounced, in general terms, to be 'bizarre and perplexing in the extreme.' Possibly, too, some of our English physicists had not formed a duly high opinion of the skill of their American fellow-workers. But, be this as it may, certainly the American astronomers. 
were somewhat cavalierly treated, and the acceptance of their observations was postponed until such time as European astronomers should have been able to confirm those perplexing results.

The chief interest of the eclipse of December 1870 undoubtedly attaches to this special question. Some few may have felt doubtful whether the observations to be then made might not serve to overthrow or to establish the theory that the corona is a solar appendage. But it is no secret that the minds of all astronomers capable of weighing the evidence had been made up on this point long before the expeditions started. The question, however, whether the American observations would be confirmed or not was one on which grave doubts prevailed in many quarters. For myself I must admit that these doubts had seemed to me to involve an unjust disparagement of the skill of American men of science, who have again and again proved themselves the equals of the best European observers in judgment and acumen, and often their superiors in energy. A careful study of the accounts given by the heads of the different observing parties, and more especially of the voluminous records in Commodore Sand's ' Reports of the Eclipse Observations of August 7, 1869,' had convinced me that future observations would confirm the statements made by the spectroscopic observers of the American eclipse.

This has, in effect, happened. The first fruits of the eclipse expeditions of 1870 may be said to consist in this important fact-that the observations made in 
1869, bizarre and perplexing though they seemed, and doubtful as many had held them to be, are shown to be exact and trustworthy.

From the powerful observing party which was stationed at Oran we have no results. A clouded sky sufficed to render vain the hopes which had been formed when it was known that Dr. Huggins, the Herschel of the spectroscope, and those profound students of nature, Tyndall and Gladstone, had united their forces, and, with other able allies, were to seek one of the most promising stations along the whole course of the moon's shadow.

But from Spain and Sicily, whither the two other parties of observers had betaken themselves, we have no doubtful intelligence on this special point. From Spain we have (at the present writing) the fullest details. As on former occasions, some observers failed to see the bright ines. This failure is not remarkable when the difficult nature of the observation is considered. It has been shown, indeed, that a certain increase in the quantity of light admitted to form the spectrum would suffice to obliterate the lines altogether from view, while rendering the rainbow-tinted background considerably brighter. Negative evidence in this case proves nothing. The great question was whether reliable positive evidence would be obtained. Fortunately, two observers succeeded in answering this question in a manner there could be no mistaking. Father Perry, S.J., who headed the Spanish parties, thus describes the observations made by Captain 
Maclear:- 'Knowing that an unfavourable sky would render observations with a powerful spectroscope quite impracticable, I desired Captain Maclear to observe with a small direct-vision Browning spectroscope, attached to a four-inch telescope, mounted equatorially.' The spectroscope was so placed that the light coming from a portion of space outside the sun, and directed towards his centre, was under examination. Of course, while the sun's direct light was falling on the air lying in this direction, the spectroscope showed the ordinary solar spectrum, precisely as when one of these handy direct-vision spectroscopes is turned towards the sky in the daytime. But 'immediately totality commenced,' proceeds Father Perry, 'the ordinary solar spectrum was replaced by a faint diffused light and bright lines' (whose position he indicates). 'There were no dark lines-that is to say, none of those lines which are present in the solar spectrum.' Then follows the most important part of the account. The spectroscope was directed 'to a distance of about eight minutes,' or half the moon's apparent radius, from the edge of the moon's disc. 'The same lines remained visible.' ' The centre of the moon was then tried, and the bright lines were still seen, but only half as strong as before.' The spectroscope was then again directed to a point eight minutes outside the moon, and the lines were restored to their original brightness.

These results require to be considered somewhat carefully. The reader cannot fail to be surprised by the fact that, from the direction in which lay the centre 
of the moon's seemingly black disc, light of the same quality as that from the corona was received and analysed by the spectroscopist. Yet, on reflection, it will appear that this result was to have been anticipated; for since during the whole eclipse the corona continues visible, it follows that the air around and above the observer is during the whole eclipse illuminated by the corona. This illuminated air, therefore (if its light became sensible at all), would necessarily supply the same spectrum as the corona, only considerably reduced in brightness; and this, as we have seen above, is what actually happened.

But then it may be argued, if this be the case as respects this seemingly dark part of the sky, may not a portion of the light which seems to be received from the corona itself-which comes at any rate from the direction towards which the corona lies-be similarly due to atmospheric reflection? It is certain that such must, indeed, be the case; but it is also certain, from the greater brilliancy of the bright lines seen when this part of the sky is examined, that a portion of the light which produces these lines comes from the corona itself. We must, indeed, subtract a certain portion, about as much, perhaps, as is received from the direction in which the moon's dark body lies, - the balance which remains belongs to the corona itself.

We should, indeed, at this point reinforce the spectroscopic observations by those results which the telescope used in the ordinary manner supplied. We must inquire what was the apparent form, where were 
the seeming limits, of the corona, as seen on this occasion.

On these points our information is sufficiently definite, although the circumstances were by no means such as would be considered favourable for clear vision of the delicate light of the corona. 'The moment of totality approached,' says Father Perry, 'and no chance remained of even a momentary break in the cirrus that enveloped the sun and obscured most of the sonthern heavens. As the crescent became thinner, the cusps were observed first to be drawn out and then blunted, the well-known "Baily's beads" were formed, and the corona burst forth more than troenty seconds before totality. Viewed through a telescope of very moderate dimensions the spectacle was grand, but the cirrus clouds destroyed almost all the grandeur of the effect for the naked eye. Mr. Browne, of Wadham College, Oxford, noticed that the corona was perfectly free from striation, outline distinct, and approximately quadrilateral, bat extending furthest in the direction of first contact. The brightest part of the corona appeared to the unassisted eye to be scarcely more than one-tenth of the sun's diameter, fading rapidly when one-fifth, bat being still clearly visible at seven-ighths. Some observed two curved rays, but the general appearance was that of a diffused light, interrupted in four places distinctly, and in a fifth faintly, by dark intervals. The corona was white, and rendered faint by the 'clonds.'

It is clear, then, that that part of the sky whence 
the light came which gare the spectrum of bright lines was visibly occupied by the corrona at the time. No question can remain then, it would seem, as to the true source of at least a lange proportion of that light. The corona itself must have supplied it.

We learn further, that at another station, near Xeres, Mr. Abbaye made similar observations.

From Sicily we have not such definite statements. But the telegram received from Mr. Lockyer amnounces in general terms that the American observations of 1869 have been confirmed; and the force of this announcement is somewhat strengthened by the circumstance that Mr. Lockyer had been disposed to believe that the American astronomers had been deceired in 1869.

In comparison with this result, that the light of the corona gives a spectrum of bright lines-or rather a mixed spectrum in which bright lines are seen superposed on a rainbow-tinted background-all the other observations made during the late eclipse sink into relative insignificance. Let us briefly consider what conclusions may be deduced from the observed facts, premising that the doubts which have been so long allowed to rest on the statements made by the American observers in 1869 , ought not to prevent as from assigning to them the full credit of attaining to the discorery of these bright lines.

In the first place, the rainbow-tinted spectrum implies that a portion of the coronal light comes from incandescent solid or liquid matter. It is barely 
possible, of course, that there are in reality dark lines across this rainbow-tinted streak, but that these lines remain undetected owing to the extreme faintness of the spectrum itself across which they lie. If we adopted this view we might assume that the corona shone in part at least, by reffecting the sun's light. As far as the evidence goes, however, we have the theory presented as, on the whole, more probable, that the matter of which the corona consists is, in large part, incandescent through intensity of heat. It is difficult to suppose that such skilful observers as have studied the coronal spectrum would have failed to detect dark lines, had any existed. On the other hand, we have à priori reasons for believing that the matter of the corona, or at least of that part which has been analysed with the spectroscope, must be intensely beated. A portion of the corona which appears to lie but eight minutes from the sun's edge, must lie in reality so close to his orb that the sun, instead of appearing as a disc but about half a degree in width, would seem nearly ninety degrees wide, and the amount of heat received from him would be many thousand times greater than that received on the hottest day of a tropical summer. We can form an opinion of the effect of such heat as this, in the same way that Sir John Herschel estimated the heat received by the great comet of 1843, when nearest to the sun. 'To form some practical idea of this,' he writes, 'we may compare it with what is recorded of Parker's great lens, whosediameter was $32 \frac{1}{2}$ inches, and focal length 6 feet 8 . 
inches. The effect of this, supposing all the light and heat transmitted, and the focal concentration perfect (both conditions being very imperfectly satisfied), would be to enlarge the sun's effective angular diameter to about $23 \frac{1}{2}$ degrees.' This, he shows, would give a heat 1,915 times greater than that received by the earth, 'and when increased' seven-fold, as was usually the case, would give 13,400 times' the heat received by the earth. The heat received by the matter of the corona would be fully twice as great as this; 'yet,' says Sir John, ' the lens, so used, melted cornelian, agate, and rock crystal.'

And here a somewhat curious subject presents itself for consideration - a subject which has not hitherto, so far as I know, been very carefully attended to. It may seem that material so diffused and tenuous as "that of the corona would be altogether invisible, however intensely heated and illuminated. For, beyond question, the actual quantity of matter in the corona must be indefinitely small by comparison with the space which this object fills. It may be doubted, indeed, whether all the matter in a portion of the corona as large as our earth might not be outweighed by halfa-dozen peppercorns.

But so far as the visibility of the corona is concerned, the extremely fine division to which its, material substance is almost certainly subject, would tend to compensate for the quantitative minuteness of that material. A very simple illustration will explain my meaning. This earth of ours reflects a certain amount 
of sunlight towards the inner planets, Venus and Mercury. Now suppose the earth were divided into eight equal parts, and each fashioned into a globe. The eight globes would each have a diameter half the earth's present diameter, and each would reflect onefourth of the light which the earth now reflects. The eight then would reflect altogether twice as much light as the earth actually reflects; and yet their combined bulk would only equal hers. If each of these eight globes were divided into eight others, four times as much light would be reflected as the earth now reflects. And if the division were continued until the several globes were reduced to mere grains, and these grains were well spread out, the quantity of sunlight which the cloud of grains would intercept and reflect towards the interior planets would exceed many millionfold that which the earth actually reflects. In like manner, an incandescent globe, if divided into myriads of minute incandescent globes, would supply much more light than in its original condition.

So in the case of the coronal matter. Assuming it to consist of myriads of indefinitely minute particles, very widely dispersed, it would be capable of emitting and reflecting a quantity of light altogether disproportioned to its actual weight regarding it as a whole.

But when we consider the spectrum of bright lines given by the corona, the case no longer remains altogether so simple. One cannot very readily accept the opinion of Professor Harkness, that this portion of the coronal light comes from iron existing in the state 
of vapour; for, although it is exceedingly probable that iron forms one of the chief constituents of the coronal substance, yet, in the first place, we have no reason for believing that a degree of heat intense enough to vaporize iron would exist where we see the corona; and, in the second, other elements must also be present in the coronal substance, and they also would be vaporized, whereas we find none of the lines due to other known elements.

The idea suggested by Professor Young and others seems more likely to be the correct explanation of the matter. For bizarre and fanciful as the idea may seem that the corona is a perpetual solar aurora, it must not be forgotten that General Sabine and Dr. Stewart propounded, some years since, in explanation of known terrestrial phenomena, the theory that the coloured prominences are solar auroras. This idea has been shown, indeed, to be erroneous, but the reasoning on which it was based was sufficiently sound, and the observed facts would be equally well explained by supposing the corona, instead of the prominences, to form a perpetual solar aurora.

When we remember that the zodiacal light-a phenomenon which holds a position midway between the terrestrial aurora and the solar corona-has been shown to give a spectrum closely resembling both the auroral and the coronal spectra, the idea does certainly seem encouraged that all three phenomena are intimately associated. We might thus not unreasonably regard the zodiacal light as the outer and very much 
fainter part of the corona, the two together forming a perpetual solar aurora; and in this way we should begin to see the means of explaining the remarkable and undoubted fact that the displays of our terrestrial auroras are associated in a most intimate manner with the condition of the solar surface. For we should be led to regard the recurrence of our auroras as a manifestation of the same sort of solar action which is more constantly at work amidst the materials constituting the corona and the zodiacal light.

This view leaves unexplained the bright lines of the coronal spectrum. But as we have every reason for regarding the auroral light as an electrical phenomenon, and the bright lines in the auroral spectrum as therefore not due to the presence of vast quantities of glowing vapour, we may extend the same interpretation to the coronal spectrum. In laboratory experiments, when the electric spark passes between two iron points, its spectrum shows the lines belonging to vaporized iron, and yet the quantity of iron vaporized by the spark is almost infinitesimally minute. And similarly, if we regard the corona as an electrical phenomenon, we get over the difficulty. which opposes itself to Professor Harkness' theory, that a large proportion of the corona consists of the luminous vapour of iron.

The general result would seem confirmatory of these views, according to which the real origin of the coronal light is to be sought in the millions of meteor-systems which undoubtedly circle round the sun, many of them passing (when in perihelion) very close to his globe. 
These meteor systems have been shown to be associated with comets, though, as yet, the exact nature of the association is little understood. From what we have learned respecting them, we should expect the sun during eclipse to be surrounded as with a crown or glory of light, due to the illumination of the mixed cometic and meteoric matter. We should also, for like reason, expect to find a faint glow along that very region of the heavens where the zodiacal light is seen. When we add to these considerations the circumstance that all other theories of the corona and zodiacal light appear to be disposed of by the evidence at present in our hands, it would certainly seem that we have fair reason for regarding the interpretation here set forth as at least, in the main, the true one. Many details may yet remain to be considered; many peculiarities, both of the corona and of meteoric systems, may remain to be ascertained : and, fortunately, the means are not wanting for fruitful research into both subjects. But this general view seems demonstrated, that the facts recently ascertained by astronomers respecting meteoric systems on the one hand, and the corona on the other, are closely related together. It is highly probable, also, that the association between the two orders of facts will become more and more clearly apparent with the further progress of observation and of that careful analysis of observation which alone educes its true value.

Cornhill Magazine, January 1871. 
74 LIGHT SCIENCE FOR LEISURE HOURS.

\section{YET A YEAR LATER.}

THE eclipse of December 1871 occupies a somewhat exceptional position. It is the last of a series of important total eclipses of the sun following each other at comparatively short intervals, and each (thus far) distinguished by some noteworthy accession to our knowledge. Between the eclipse of June 1860 and that of August 1868 very little was added to our information respecting those solar phenomena which are visible during total eclipses. Of course the sun was totally eclipsed more than once during that interval, but either the circumstances of such eclipses were unfavourable, or else the regions where they could be viewed were so situated as to preclude the possibility of forming well-organised observing parties. The great Indian eclipse of August 1868 terminated this long period of inaction. Then came the important American eclipse of August 1869 ; and next, the socalled Mediterranean eclipse of December 1870. During all these eclipses very striking discoveries were made. It remains to be seen whether the eclipse of the present month will supply the means of so supplementing those discoveries as to satisfy the craving minds of astronomers during the next twentyeight months. It is in any case certain that during the interval just named no eclipses will occur which will be worth the trouble of observing in the systematic 
and expensive manner justified by the circumstances of the recent eclipses.

My present purpose is chiefly to indicate the nature of the hopes entertained by astronomers respecting the approaching eclipse, as well as the position to which the observation of the eclipsed sun has already led the students of solar physics. But the opportunity is a favourable one for a brief consideration of the laws according to which solar eclipses succeed each other.

We are apt to regard the prediction of eclipses, and eclipses generally, as among the most mysterious of all the subjects with which astronomers have to deal, and in one view of the matter this is not very far from the truth. Certainly the processes by which the exact circumstances of eclipses are determined years before they occur, are among the most surprising developments of the powers of the human mind which the whole body of science makes us acquainted with. But the general laws of eclipses are not particularly abstrusecertainly not so abstruse as to account for the perplexity with which the subject is very commonly regarded.

I am inclined sometimes to think that our books on astronomy are not always strictly fair to their readers. Something must always be taken for granted in popular treatises, while other matters are selected for special consideration. But it seems to me, with all deference to the authors of our original treatises on astronomy, that they sometimes discuss far too thoroughly certain matters which the general reader cares very little about, while, on the other hand, they 
occasionally take for granted and leave unexplained just those matters which the student is best able, as well as most anxious, to comprehend.

Eclipses certainly seem to me to be a case in point. There is something amusing-so at least I conceivein the elaborate care with which the student of the noblest of all sciences is informed that an opaque body can cast a shadow, and that this shadow will have such and such characteristics. I am not here speaking of elementary treatises : it is reasonable enough, perhaps, in a first book for children to explain that "when the moon stops the sun's light its shadow falls on a part of the earth,' and that ' the people who live on that particular part of the earth where the shadow falls cannot see the sun because the moon is in the way.' This is very pleasing and instructive for very small people; but when in treatises of a higher class the student is gravely informed of these things, as though they involved entirely new and striking conceptions, the idea is suggested that astronomers think but lightly of the capacity of those who chance not to have made astronomy their chief subject of inquiry.

On the other hand, the points about which most readers would care to hear something are commonly left untouched. Scarcely any reader of the usual explanation of eclipses fails to feel interested in the question of the laws according to which the moon comes between the sun and the earth, or the earth between the sun and the moon. The student feels that it may be very well to show him the consequences which follow when 
these bodies assume particular positions; but that he would also like to know a little about the causes of their becoming so placed, as well as of the laws according to which the sequence of such events is determined.

We are thus led to a mode of considering the subject which is very generally useful in the study of astronomy. I cannot, indeed, too earnestly recommend the student of the science to employ this method at every opportunity. It consists in imagining oneself placed at some suitable standpoint whence all the movements of such and such celestial bodies may be watched.

In this case, the proper standpoint is the sun himself, and the bodies to be watched in imagination are the earth and moon. The student must picture to himself this earth on which we live, as a small globe circling around his standpoint once in a year. $\mathrm{He}$ must conceive this globe as no larger in appearance than any one of the planets as seen from the earth. $\mathrm{He}$ would, indeed, require a good telescope to see the earth (from his place on the sun) actually as a globe. Now let him further conceive that around this small globe a much smaller orb is circling once in rather more than four weeks; but that the direction in which he looks at the circular path of the smaller orb is always such that this orb seems to travel backwards and forwards across or close past the larger one. To show exactly how long this path would look as seen from the sun, as well as to illustrate other points of 
interest connected with this explanation, the following process may be employed. Let the reader draw a circle ten and three-quarter inches in diameter to represent the sun or moon as we see these orbs. At the centre of this circle draw a small one, one-tenth of an inch in diameter; this will represent the earth as seen from the sun. Three inches from this small circle set another, a fortieth of an inch in diameter; this will represent the moon as seen from the sun when at her greatest range of distance from the earth. Exactly on the opposite side of the little circle representing the earth, and three inches from that circle, set another little picture of the moon ; this represents the moon as seen from the sun when at her greatest range of distance from the earth on the other side. The observer in the sun would see the moon pass backwards and forwards from one position to the other in rather more than four weeks. In thus moving backwards and forwards the moon passes always close (in appearance) to the earth, but sometimes closer than at others, and sometimes right across or right behind the earth's face. The path, in fact, opens out into an oval whose greatest width, on our scale, is slightly more than five-tenths of an inch, then closes up, then opens out to the same degree, only tilted the other way, then closes up again, and so on continually, while the earth all the time is circling round the observer's standpoint once in a year, and the moon round her path (thus varying in aspect) ${ }^{\mathrm{I}}$ once in

1 Of course the path is not a real entity, and could not therefore be seen, as supposed. It is convenient, however, to regard it as such. 
twenty-nine and a half days. Speaking roughly, we may say that once a fortnight the imagined observer in the sun would see the moon crossing the earth's place. He would always see the moon close to the earth, since we have seen that the whole length of the moon's path, as seen from the sun, is much less than the breadth of the sun's globe as we see it; but twice in a month the moon would be very close by the earth.

Now our observer in the sun would see that the moan's path passed from its greatest opening to a seeming line, and thence to its greatest opening again (but with opposite tilt) in five months and about three weeks; passing back to a seeming line and to its original opening again, in all respects as at first, in the same time. Eleven months and eleven days complete the whole set of changes. When the path seemed most open the moon would not at any time actually cross the earth's face, or pass actually behind it. In other words, the moon would neither hide any part of the earth from the sun nor be hidden by the earth. Hiding any parts of the earth from the sun means obviously eclipsing the sun as viewed from those parts of the earth; while to say the moon is hidden from the sun by the earth means (no less obviously) that

We may thus compare it to the outer rim of Saturn's ring-system ; and precisely as we see that ring-system closing up and opening out systematically in the course of about twenty-nine years, so certainly an observer on the sun, watching our moon's course, would find her path opening out and closing up systematically in the course of eleren months eleven days, the seeming length of the path remaining appreciably unchanged, and about equal to three-fifths of the seeming diameter of the sun as seen from the earth. 
the moon is thrown into shadow, or eclipsed. So that when the moon's path, as seen from the sun, is most open -forming then a long oval-there can be no eclipses either of the sun or moon. But when this path has in appearance closed up to a line, or nearly to a line, the moon can no longer pass by the earth (as viewed from the sun) without actually crossing the earth's disc or passing actually behind that disc. So long as this state of things lasts there must be an eclipse whenever the moon's backward and forward motion carries her past the earth. We have seen that the moon's path had this aspect, or is closed up into a straight line, as seen from the sun, at intervals of about five months and three weeks. For rather more than a month the path is sufficiently closed for eclipses to occur. I have suggested for these occasions the title of 'eclipse months.' To show how they succeed each other, take the following illustrative instance:-Let January in any year be an eclipse month, the middle of January being the time when the moon's path appears closed up into a line as seen from the sun. Then five months and three weeks later, or about July 6, the path is again closed up into a line as seen from the sun; and a period of rather more than a month, having this date for its middleor from about June 22 to about July 23 -is again an 'eclipse month.' Passing on from July 6, we reach in five months and three weeks the date December 27, which is the middle of the next ' eclipse month.' And so on continually.

Other matters connected with the recurrence and 
peculiarities of these 'eclipse months' belong, or should belong, to treatises on astronomy. What has been said above suffices for my present purposewhich is to explain the sequence of the late eclipses. It will be observed that about eleven months and eleven days separate an eclipse month in one year from the corresponding eclipse month in the next. We thus see why the great Indian eclipse of August 1868 had its analogues, so to speak, in the total eclipse of August 29 in the preceding year, and in the American eclipse of August 7, 1869. These three eclipses, occurring eleven days earlier in each succeeding year, were all three total. But the series did not end with the eclipse of August 1869. On July 2\%, 1870 (again eleven days earlier) there was an eclipse of the sun. It was, however, only a partial one, and closed the series.

Now the eclipse of the present month belongs to another series. It will be remembered by every one that there was an eclipse on December 22, 1870; that eclipse was the first of the series to which the approaching eclipse belongs. This series, like the former, includes four eclipses. In December 1870, the moon, as seen from the sun, crossed the earth's face near its northern edge. In the eclipse of December $12, \cdot 1871$, the moon, as supposed to be seen from the sun, will pass slightly to the north of the middle point of the earth's face. ${ }^{1}$ Thus the eclipse will be

1 It is a singular circumstance that the earth will present almost exactly the same face towards the sun at the moment of central eclipse III. 
more important than that of last year, and the length of the actual track of the moon's shadow considerably greater. The third eclipse of the series will occur on November 30,1872 . In one respect it will be one of the most remarkable ever recorded; for it must be described as at once an annular and a total eclipse of the sun. This is readily explained, though the occurrence is altogether exceptional. The reader is aware that the point of the moon's conical shadow sometimes extends beyond and sometimes falls short of the earth. In the former case an eclipse is total, in the latter it is annular. But in the eclipse of November 30, 1872, the apex of the shadow falls short of the earth's surface at the beginning of the eclipse; it encounters the earth as the shadow-track passed onwards towards the bulging central part of the earth's illuminated hemisphere; and presently, towards the close of the eclipse, falls again short of the earth's surface. So that there are two points on the earth's surface where, on November 30, 1872, the eclipse will be exactly total, the moon just hiding the sun and no more, and only for a single instant. The totality will nowhere last more than about three-quarters of a minute; and as

on the 12th inst., as at the middle of the transit of Venus, on December 8,1874 . The fifteen pictures of the rotating earth, in Plate VIII. of my treatise on the sun, illustrate the approaching eclipse as exactly as though drawn for the purpose. The first shows the earth's face as seen from the sun just before the moon's passage begins; the next thirteen show the earth's face at successive interrals of a quarter of an hour during the progress of the eclipse; and the last shows the earth's face as seen from the sun just after the moon has passed off that face. 
the place where this will happen lies very far south in the Pacific Ocean, it is not likely that any observer will witness this eclipse. It is, however, the most considerable solar eclipse of the year 1872. The last eclipse of the series occurs on November 19, 1873, and, like the last of the former series, it is altogether unimportant. The moon, as supposed to be seen from the sun, will just graze the most southerly part of the earth's disc. 'The circumstances of the eclipse are such,' says the Nautical Almanac, 'that a map has not bẻen considered requisite.' There will be no total. solar eclipse at, all in 1873.

Not until A\}ril 16, 1874, will any total eclipse worth observing take place, after the eclipse of the present month. Nor are the circumstances of the eclipse of 1874 such as to encourage favourable hopes that much will be learned during its progress. On April 6,1875 , there will be, I believe, a much mare important.eclipse visible (as I judge from a rough calculation) in Africa; but I shall probably be excused from entering into an exact calculation of its circumstances, more especially as the Nautical Almanac for 1875 will, I believe, be published before this essay appears.

It will be inferred that a considerable degree of interest is attached by astronomers to the eclipse of the present month, followed as it will be by two years and four months during which there will be no solar eclipses worthy of special observation.

Although the eclipse of December 12 is not nearly 
so favourable for observational purposes as the great Indian eclipse of 1868 , yet there is a considerable variety as respects the choice of stations. In fact, there are no less than four distinct sections of the moon's shadow-track to which it has been judged advisable to send observers. The track crosses the southern extremity of the Indian peninsula, and along this part of its course there will probably be several observing parties, the arrangements being superintended by Mr. Pogson, the Government Astronomer at Madras, and by Colonel Tennant and Captain Herschel, both known to fame through their observations during the great eclipse of 1868. Thence the shadow-track passes to the northern part of Ceylon, and along this part of its course the English eclipse expedition will be stationed. It will probably be in the remembrance of most of my readers that the English Government granted (several months back) the sum of $2,000 l$., as well as transport and the means of camping, for an expedition to Ceylon. It was hoped that Professor Stokes would have been able to take charge of this expedition; but these hopes were disappointed. Mr. Lockyer, however, has been able to give his services, and doubtless the expedition will be a highly effective one. The shadowtrack passes from Ceylon to Java, where a French party under M. Janssen will be stationed. Lastly, the shadow-track passes to the northern part of the Australian continent, and a strong observing party has proceeded from Sydney and Melbourne to the stations. along this part of the shadow's course. 
The totality will last longest in North Australia, where its duration will be more than four minutes, or nearly two minutes longer than the duration of the eclipse of last year at the best stations. In Java the totality will last more than three minutes. In Ceylon the duration of totality will barely exceed by a few seconds the duration of totality last December. A somewhat curious mistake was made on this point in a scientific journal. Mr. Hind, in his first and comparatively rough estimate of the course traversed by the moon's shadow, had placed Trincomalee on the border of the track, so that the duration of totality at Trincomalee would have been very short. But after his final and more elaborate calculation, he set Trincomalee close to the centre of the shadow-track, with a duration of total obscuration amounting to two and a half minutes. Strangely enough the increase of the estimated duration was alone noticed by the writer of an article in Nature, and it was reasoned that since the duration is so considerable at Trincomalee on the border of the track, it must be very much greater at places on the centre of the track. I need scarcely point out that this inference was unwarranted. In fact, the duration of totality can never under any circumstances be considerable for places close to the border of the shadow-track. ${ }^{1}$ In southern India the eclipse will last about as long as the eclipse of last year at the best stations.

'A somewhat similar mistake occurred in 1870 whereby the Sicilian .eclipse party formed too sanguine expectations of the duration of totality in that island. 
It cannot be doubted that the observers in 1871 will have a much more difficult task than those who have added so importantly to our knowledge during the eclipses of the last three years. This will appear on a brief consideration of the progress and present position of the problem with which the observers are to deal.

In 1868 , the observers of the great Indian eclipse discovered that the solar prominences are vast masses of glowing vapour, hydrogen being the chief constituent of these marvellous objects. But the solar corona, that glory of light which appears around and beyond the coloured prominences, did not at that time receive its interpretation. In 1869, the American observers directed their chief attention to this beautiful phenomenon; and they were singularly successful in their observations. One result of a very remarkable character was obtained by several observers. The light of the corona when analysed in the spectroscope was found to be in large part monochromatic, the coronal spectrum showing one bright line. Now the reader is doubtless aware that in spectrum analysis the essential point is to determine where any bright or dark lines may lie along the range of that rainbow-tinted streak which we call the solar spectrum. In this instance the position of the bright line has been most satisfactorily determined by a very skilful spectroscopist, Professor Young, of America. The line agrees in position with one of the lines in the spectrum of iron, a line also seen in the spectrum of the aurora borealis. 
But the spectrum of iron contains upwards of 400 lines, while even the simpler spectrum of the aurora contains several lines; that of the corona, on the other hand, has not been proved to contain any other bright lines except the one just mentioned. Others have been suspected, but the degree of their brightness has not been such as to prove beyond all possibility of question that. they belong to the solar corona.

However, as Professor Young remarks on this point. (writing in 1871), ' considered as a demonstration of self-luminosity one bright line is just as conclusive as. many.'

It was in fact demonstrated by this observation alone that the corona, for a considerable part at least. of its extension, is a self-luminous object. 'Nor can there be any doubt,' I may add with Professor Young, ' as to the location of the self-luminous matter. It cannot be in our atmosphere, for no possible reason can be assigned why the particular molecules of the air that happen to lie near the lines which join the eye of the observer with the edge of the moon should become luminous rather than others in a different portion of the sky. Nor can it be at the moon; otherwise, of course, it would always be visible round her disc.' 'Accordingly,' he adds, 'it is now universally, I think I may say, acknowledged that one important element of the corona consists of a solar envelope of glowing gas reaching to a considerable elevation. Mr. Lockyer, who is still disposed to assign to the solar element of the corona a lower relative importance 
than most other astronomers, concedes a thickness of from six to ten minutes' - that is, from a fifth to a third of the solar diameter.

This, as I have said, was written by Professor Young in 1871 , but before a certain most important fact had come to his knowledge, which without at all affecting what he here puts forward, renders it possible to say much more as to the real extension of the corona.

We have seen that a certain object, surrounding the sun on all sides to a distance of from 160,000 miles to 290,000 miles from his surface, is demonstrably a self-luminous envelope. It was to this envelope, or perhaps rather to its brighter portion as seen from the earth, that some proposed to assign the barbarous name 'leucosphere,' to distinguish it from the bright layer of prominence matter close by the sun, which is called the sierra, or chromatosphere. But the visible extension of the corona is greater yet, and before the eclipse of 1870 doubts still existed as to the actual extent of that solar corona, which all had now begun to recognise as a real entity. That some portion of the light seen around the sun during total eclipse is in reality only due to the illumination of our own atmosphere is altogether beyond question. It is true, indeed, as was pointed out by Professors Young and Harkness, Dr. Curtis, and myself, that none of the coronal light, for several degrees from the sun's place, can be solar light reflected by our atmosphere, as had been mistakenly supposed; but it is no less certain 
that our atmosphere is illuminated not merely in directions lying close up to the moon's edge, but even towards the body of the moon herself, by the light of the coloured prominences and of the real solar corona. The observer himself sees these luminous objects during totality, and therefore the air all round him must be illuminated by them. ${ }^{1}$

Now here a question of extreme delicacy arises. The true solar corona undoubtedly grows fainter and fainter with increased extension from the sun. That is, if we could see the corona from some point raised above the earth's atmosphere, so that no terrestrial illumination could deceive us, we should see the corona gradually diminishing in lustre with distance from the sun, until at last it became too faint to be discerned at all. On the contrary, the illumination of our atmosphere during totality must necessarily increase with distance from the direction of the eclipsed sun.

1 One cannot but be surprised at the stress which was laid by some, soon after the eclipse of last December, on the fact that even directly towards the moon's place, light was received which the spectroscope showed to be similar in character to that of the bright inner portion of the corona. Not only was the fact dwelt on repeatedly as a proof that the corona lies on our side of the moon, but it was commended to my own special attention as a proof that I had been mistaken in urging before the eclipse of 1870 that the corona is demonstrably a solar appendage. In the very paper in which $\mathrm{I}$ urged this view before the Royal Astronomical Society, on March 11, 1870, I pointed out that our air must be illuminated towards the moon's place by the light of all the visible solar appendages-as the prominences, chromatosphere, and corona-as well as by reflected earth-light. My words were sufficiently distinct. They ran as follows:- The light from all these sources should extend over the moon's disc, since it would illuminate the air between the observer and the moon's body.' 
This is obvious, because those molecules of the air which lie directly towards the moon's place are themselves suffering total eclipse from the sun's direct light, and are illuminated by a rather less proportion of prominence and coronal light than the observer himself, whereas those molecules which lie in directions far removed from the place of the eclipsed sun are suffering either but a partial eclipse, or else, though their eclipse be total, they are yet illuminated by more lustrous portions of the corona and prominencematter. So that so far as atmospheric glare alone is concerned, we should have, as I wrote in March 1870 , a relatively 'dark region around the eclipsed sun and a gradual increase of light with distance from him.'

The question which arises here, then, is this-at what distance from the eclipsed sun has the light of the solar corona so diminished, and that of the atmospheric glare so increased, that the latter light predominates over the former. This question is not only exceedingly nice, but, as actually stated, it is wholly unanswerable, unless as a matter of fact the real solar corona has definite limits, recognisable perhaps by more refined methods of observation than have yet been applied.

But although it is unlikely that the utmost actual extension of the corona can be determined by means of such appliances as are at present available, yet it was possible last December to demonstrate the extension of the corona to a distance far exceeding the six 
or ten minutes acknowledged by those who had once sought to reason away the corona almost wholly. It is clear that if any definite coronal feature extending more than ten minutes from the place of the eclipsed sun could be seen at stations far apart, then beyond all question that feature would be shown to be extra terrestrial. For instance, it could not possibly be imagined that some peculiarity in the air over Syracuse could reproduce a feature of this sort precisely as it appeared to the observers near $\mathrm{Xerez}$, owing to a peculiarity of the air over this station.

Now, soon after the eclipse occurred, it was announced that the observers in Spain had recognised a peculiar gap, shaped like a letter $\mathrm{V}$, in the lower portion of the corona-on the left hand. This gap was pictured and described to me by my friend, $\mathrm{Mr}$. W. H. H. Hudson, M.A., and Fellow of St. John's College, Cambridge, before any of the other accounts had come under my notice; and it was with some interest that I awaited the January meeting of the Royal Astronomical Society, before which the records of the observers in Spain were to be presented. At that meeting a picture was exhibited by Lieutenant Brown, in which this $\mathrm{V}$-shaped gap was a very prominent feature. But in the discussion which ensued after Lieutenant Brown's paper had been read, Mr. Hudson remarked that the gap had seemed somewhat larger to him, on which Lieutenant Brown admitted that perhaps the size of the gap had not been quite adequately presented in his drawing. 
After the meeting a photograph, taken during the eclipse by Mr. Willard, of America, was shown to a few of those present. Why the picture was not exhibited and described at the meeting itself I do not know. Probably the description was reserved for American societies. But whatever the cause, it is certain that if the picture had been shown earlier, some doubts which were expressed respecting the real nature of the corona would have been obviated. For there, in the photograph, and occupying the precise position described to me much earlier by Mr. Hudson, and publicly described and pictured by Lieutenant Brown and others, was this V-shaped gap.

Mr. Willard's photograph was taken at a station near Xerez, so that all that has hitherto been said relates to Spanish observations. To complete this portion of the evidence, I quote the following passage from an interesting account of the eclipse by one of the observers in Spain. It is extracted from the English Mechanic for January 27, 1871. 'The corona proper, or glory, or radiated corona-as it is variously called-extended a distance of almost the moon's diameter from the moon's edge, but not equally in every direction. It had a greater extension in four directions, at the extremities of two diameters at right angles to each other, so as to give it the shape, roughly speaking, of a square with rounded corners. It was broken in parts, and notably by one decided V-shaped gap. This was observed, not only by one party, but at three stations, San Antonio, Xerez, and La Maria 
Louisa, which form a triangle, each of whose sides is five or six miles in length.'

But in the meantime news had been received from Sicily which conveyed the unpleasing impression that the observations there had been all but complete failures. In particular it was supposed that Mr. Brothers, who had the management of the photographic department there, had been unable to obtain any useful results-since no mention had yet been made of his success. I was indeed as much surprised as pleased, when I received a letter from him announcing that he had secured five photographs of the corona, in one of which the corona appeared ' as it had never been seen on glass before.' It will be conceived that I awaited with great interest even the first rough sketch of the corona as there pictured. If the $\mathrm{V}$ shaped gap appeared in such sketch, the conclusion would be inevitable that a real solar appendage exists having an extension at least equal to that indicated by the bounding edges of the gap-that is, an extension of at least 600,000 miles. If, on the other hand, that well-marked peculiarity failed to present itself, the inference would be that it does not exist in the photograph, and that, therefore, the seeming gap was due to some peculiarity of the atmospheric illumination at the Spanish stations. It would not, in this case, be by any means demonstrated that the sun has no appendage reaching so far as five or six hundred thousand miles from the sun's surface, but it would be quite certain that the evidence given by the $\mathrm{V}$ - 
shaped gap could not be accepted as demonstrative or even trustworthy. The presence of the V-shaped gap in Mr. Brothers's photograph would supply an argument positive and final; its absence would supply a negative argument, proving nothing, however, and leaving the matter much where it stood before the eclipse took place.

The first sketch I received was contained in a hasty note from Mr. Brothers, written soon after his arrival in England. I was surprised, and, to say the truth, somewhat disappointed, to find that the V-shaped gap was not shown, as in the Spanish pictures. There were several gaps, but not one in the lower left-hand portion of the corona. But in the next letter which I received, Mr. Brothers intimated that the sketch was only intended to show the general aspect of the corona - to show its radiated structure-and that, in fact, he had not copied the sketch from the photograph, the negative not being as yet unpacked. Some days elapsed before a drawing made from the photograph was sent to me. In this drawing the V-shaped gap was not only presented in the same place as in the Spanish views, but, as in them, it formed the most remarkable feature of the corona. Soon after, photographs taken directly from Mr. Brothers's negative were in the hands of all who took interest in the subject, and there-pictured by the corona itself-was the gap on which so much was held to depend. All possibility of mistake as to the reality of the agreement between this gap and the gap shown in the American 
photograph was removed by the circumstance that two other gaps, less marked but still recognisable, appeared in both photographs.

I have dwelt somewhat at length on this V-shaped gap, because it is in reality of extreme importance. On no former occasion had any distinctive feature of the corona been unmistakably recognised at stations far apart. It happened strangely that on the first occasion upon which the corona was successfully photographed, a very remarkable and characteristic peculiarity was presented by the corona. Favourable as are the circumstances of the approaching eclipse, it is not by any means certain that the photographs taken at distant stations will be so well suited for comparison as those taken during the eclipse of last year. So that it is well to set store by the great fact which was established by the observers of the latter eclipse. The following words taken from a letter addressed to Mr. Brothers by Sir John Herschel, serve to indicate the importance which he attached to the photographic racords of the V-shaped gap:- 'Assuredly,' he wrote, 'the decidedly marked notch or bay in both photographs' (those taken at Cadiz and Syracuse) ' agreeing so perfectly in situation (marked so definitely by its occurrence just opposite the middle point between two unmistakable red prominences) is evidence not to be refused, of its extra-atmospheric origin. ${ }^{1}$... A

I The omitted words relate to the absence of any signs which could show the corona to be a phenomenon produced within the space separating the earth from the moon. On this point, further, I may remark that I had occasion to submit to Sir John Herschel certain considera- 
terrestrial atmospheric origin is quite out of the. question.'

And here, in passing, I may venture to note as somewhat surprising-in the presence of such an opinion, announced publicly before the highest astronomical tribunal of this kingdom-the statement made by the President of the last meeting of the British Association, that the observations during the eclipse of 1870 proved the terrestrial atmospheric origin of at least the principal portion of the coronal light. It is amusing to read this now, when the last shred of the terrestrial theory has vanished. Even if we rejected the positive evidence obtained during that eclipse, and even if we regarded Herschel's opinion as of no weight whatever, it would still be impossible to point to a single fact discovered in December 1871 which tended to confirm the atmospheric theory. Facts were noticed then, as facts had been noticed before, which at a first view seem to suggest a terrestrial origin of the coronal phenomena; but undoubtedly none of those facts were novel. Every circumstance that was new to astronomers was in favour of the extra-terrestrial origin, which, as we have seen, Sir John Herschel tions relating to a theory that the radiations of the corona are produced by the passage of the solar rays past the moon's edge, through dispersed meteoric matter between the earth and the moon. I submitted, amongst other matters, this question to the great astronomer-Whether the light due to the illumination of this dispersed matter would not be altogether inferior in amount to the light received from the illumination of similar matter lying beyond the moon, up to and beyond the sun's place? His reply was, as I had fully expected, that undoubtedly this consideration (which he had not before noticed) rendered the lunar theory of the corona altogether untenable. 
regarded as demonstrated. It is at least unfortunate that in thus summing up the results of the costly eclipse expedition of December 1870, Sir W. Thomson did not mention what particular discovery then made seemed to his judgment to demonstrate the terrestrial origin (in the main) of the coronal phenomena. One can understand why Professor Tait, after hearing a lecture on the general subject of solar eclipses, should have remarked that what he had just heard convinced him that the corona was of terrestrial origin; for a variety of eclipse phenomena seem at a first view to suggest the atmospheric theory as the only available explanation. Moreover, there can be no question that some of the most striking phenomena presented at the beginning and towards the close of totality are actually due to the illumination of our atmosphere at those epochs by departing rays or returning rays of direct sunlight. After a lecture chiefly devoted to the consideration of precisely such phenomena as these, and illustrated by striking pictures of such phenomena, the opinion might well be formed that the chief part of the coronal radiance is simply atmospheric. It is only on a complete survey of the subject, and especially of the evidence relating to the corona as seen in the heart of the totality, that the immense weight of evidence in favour of the real existence of the corona as a solar appendage of amazing extent is clearly recognised. But so far as could be judged by the report, Sir W. Thomson's expression of opinion related solely to the new results-the discoveries, in fact-effected last December; and it.is III. 
perplexing in the extreme to hear these results described as demonstrating the atmospheric origin of the chief portion of the corona.

The only new fact which seems in the least to countenance this remarkable statement, is the -ircumstance that the light received from the direction in which the moon's dark dise lay was found, when analysed by the spectroscope, to resemble the light received from the corona. At first sight this seems to show that the corona itself is an atmospheric phenomenon. For certainly the light received from the direction of the moon's dark disc cannot come directly from a solar appendage. And as great stress was laid on this circumstance by some, unfamiliar with what was to be expected when this light came to be examined, it seems just possible that Sir W. Thomson may have been guided by their strongly-expressed opinion.

But as a matter of fact no other result could have been expected. I had myself pointed out in March 1870 , that reflected light of precisely the observed nature must be received from the moon's direction. The air above and around the observer-including necessarily that lying towards the moon's disc-must needs be illuminated by the same coronal glory which the observer gazes upon with such wonder during totality ; and the light of that atmosphere, so illuminated, must present the same characteristics as the direct light of the corona, precisely as the light of the sky when examined with the spectroscope shows the same dark lines as the direct light of the sun. 
We have only to remember, however, that the moon looks so dark during totality as to seem perfectly black, to see how very small a part atmospheric illumination can have in producing the coronal phenomena. The light received from the direction of the moon's disc must be at least as strong as any atmospheric illumination within the region occupied by the coronal, glory; for this illumination, if we could see it alone, would be nearly uniform, while, where the moon is, we receive (over and above the atmospheric illumination) no inconsiderable amount of what astronomers call earthlight. The moon's surface, at the moment of a total eclipse, is illuminated by the earth some twelve times more brightly than the earth's surface in full moonlight. If we look at a distant hill (not forest-covered) bathed in the light of the full moon, we see that it is appreciably luminous-brighter certainly in appearance than the dark-looking disc of the moon during an eclipse. Yet the moon's disc, during eclipse, is twelve times as luminous, at least; and if all other light could be removed, we should see the moon at that time as a disc illuminated with no inconsiderable degree of brightness. Since the moon actually looks almost black-though this reflected light is reinforced by the atmospheric illumination-we cannot but admit that the atmospheric illumination alone must be very inconsiderable compared with the light even of the outer parts of the corona, which, though faint, seem by no means black.

Professor Young, of America, has reasoned similarly on this point. 'Some influence,' he says, 'our atmo- 
sphere must of course have; but remembering how much the inner portion of the coronal ring exceeds in brightness the outer, it would seem that the illumination of the lunar disc must give us an exaggerated measure of the true atmospheric effect. This illumination makes the edge of the moon only enough brighter than the centre to give it the appearance of a globe, but of almost inky blackness.' Dr. Balfour Stewart, also, in a letter addressed to Mr. Brothers, points out very clearly how insignificant relatively must be the atmospheric illumination. 'The light which reaches us in a total eclipse from the centre of the moon's disc, and which may be partly due to earthlight reflected from the moon, may be safely taken as somewhat exceeding that which can possibly be due to atmospheric glare; and inasmuch as in your photographs there is very little effect on the centre of the moon's disc, I am led to think that very little of the result obtained can be due to glare. I have here confined myself strictly to your photographs, but the principle laid down is applicable to all kinds of observations ; and I must confess that I cannot at the present moment see why the streamers, if they are caused by the atmosphere, should invariably shoot outwards, and never venture to trespass upon the moon's disc.'

The present position [December 1871] of astronomers is this. They have proved that there is a solar appendage extending to a vast distance from the sun's * surface, radiated-usually, if not always-in structure, and shining in great part with its own inherent lustre. 
The portion of the corona's substance which is thus selfluminous is gaseous. It may well be, however, that there is also a self-luminous portion in the solid or liquid condition-probably in a state of fine division. And it has been rendered all but certain that a considerable portion of the corona's light is simply sunlight reflected from solid or liquid matter in the corona. For while it is perhaps doubtful whether the solid or liquid matter is self-luminous through intensity of heat, no question remains as to the actual existence of such matter. Lastly, it seems highly probable that a portion of the coronal light has an electrical origin, like the light of our auroras.

Astronomers hope to obtain, during the eclipse of December 1871 , more satisfactory information than they have at present respecting the actual extension of the corona, as well as of the various portions of which it consists. The observers will have to discriminate between the light due to atmospheric illumination, and those fainter and more delicate portions of the real corona which have as yet not been traced to their actual limits (if they have any). It is hoped, in particular, that photographs taken at the extreme stations-those in India and Northern Australia-will so confirm the evidence first obtained from Mr. Brothers's photographs as to convince the most sceptical that the corona is not a mere atmospheric phenomenon. It may well be that spectroscopists and polariscopists will obtain some new information respecting the structure of the corona; but to effect this they will have to overcome great difficulties, owing to the way in which the light from 
our air is blended with the light from the corona. Altogether, I am disposed to believe that at this stage of our progress chief reliance is to be placed on the powers of photography. After Mr. Brothers's success during the last eleven seconds only of totality (for a cloud veiled the eclipsed sun for the first tro minutes), it may fairly be hoped that by applying his method the photographers may obtain such pictures of the corona as will throw an altogether new light on this wonderful solar appendage.

St. Paul's Magazine, December 1871.

THE ECLIPSE OF 1871.

Astronomers have seldom reaped a more abundant harvest of facts during a total eclipse (making hay, after their fashion, when the sun is not shining) than they did during the eclipse of December 12th last. To say that the anticipations which they had formed were amply fulfilled, would be to say far less than the truth. Although all hoped that important facts would be discovered, few expected so complete a success as has actually been achieved. The eclipse of 1842, when the sun's coloured prominences were first observed; that of 1860 , when they were first photographed; that of 1868 , when their real nature was first determined; and that of 1870 , when the solar corona was first photo- 
graphed-all these must be regarded as marking noteworthy epochs in the progress of eclipse researches. But not one of them surpasses, or perhaps equals in importance, the eclipse of last December, when, for the first time, the wonderful complexity and magnificence of the solar surroundings were clearly revealed.

Already in these pages the progress of solar research has been described with considerable fulness of detail; and, in particular, those questions respecting the solar corona which have for the last two years been earnestly discussed by astronomers have been dealt with at length. It will, therefore, be desirable to consider in this place, not the progress and development of our knowledge respecting the sun, but the actual position of such knowledge now, when, chiefly in consequence of the observations made during the late eclipse, we are able to speak with some degree of confidence respecting the nature of those regions which lie around the luminous centre of the planetary system.

Of the actual nature of that intensely hot and brilliant surface which the sun presents to our study, I can say but little. Astronomers are not certain even whether it is liquid or gaseous, and at present their ideas respecting the intensity of its heat are in most unsatisfactory disagreement. On the one hand we have a theory by Father Secehi, the eminent Italian astronomer, according to which the heat of the sun's surface is certainly not less than ten million degrees Centigrade, or some eighteen million degrees of the 
common thermometer (in which one hundred and eighty degrees above freezing represents the heat of boiling water). On the other hand, we have a theory maintained by Faye, St. Claire Deville, Fizeau, and many others, according to which the sun's heat does not greatly exceed that obtained in the electric light, and is certainly comparable with the heat obtained in many processes of manufacture. Indeed, according to some of the most satisfactory investigations which this subject has received, the actual heat at the sun's surface does not very greatly exceed that at which iron melts; while St. Claire Deville even asserts his belief that a degree of heat not greatly beyond that which our physicists have obtained cannot possibly be exceeded under any circumstances, either in our own sun or in any of his fellow suns.

Above the glowing photosphere, or light surface of the sun, there extends to a depth of several hundred miles the most wonderful atmospheric envelope known to astronomers. In dealing with this envelope, I am touching on the work of the recent eclipse, because, although the envelope had been recognised theoretically two years ago, and its existence demonstrated during the eclipse of December 1870, yet doubts had continued to be entertained by a few respecting the reality of this relatively shallow atmosphere. I can now, however, speak of it unquestioningly, since scarcely one of those who sought for it during the late eclipse failed to recognise its existence.

Let it be premised that, at the sun's distance, a 
depth of four or five hundred miles is reduced almost to evanescence. It corresponds, in fact, as nearly as possible, to the two-thousandth part of the sun's apparent diameter, so that it need not be regarded as at all wonderful that hitherto astronomers have failed to detect any signs of so shallow an envelope. Indeed, it is not too much to say that by the aid of the telescope alone it could never have been revealed to them. A brief consideration of its nature will show how its discovery has been rendered possible by the aid of the spectroscope.

In the lower part of our own air there is always present, in greater or less quantities, the vapour of water. This vapour rises from wet earth, from rivers, lakes, and seas, and from the wide expanse of ocean, and occupies a certain portion of the lower atmospheric strata. Thus these lower strata form, as it were, a more complex atmosphere than those above them. Close by the earth there is air and aqueous vapour, while in the higher regions there is air alone $;^{1}$ that air being, as we know, composed of a certain admixture of the two gases, oxygen and nitrogen. Now, in the case of the sun a somewhat similar arrangement exists. The lower regions of the solar atmosphere are at all times occupied by certain vapours, which ordinarily do not range to any considerable elevation, simply because they cannot remain in the form of vapour except close

1 Here I am considering only the main constituents of the atmosphere. Relatively minute quantities of other gases are ordinarily present in the upper as well as in the lower regions of the air. 
by the sun. But these vapours are such as we should be rather startled to find in our own atmosphere. We breathe the vapour of water without inconvenience, unless it is present in too great quantities; but if we could imagine for a moment that there were breathing creatures on the sun, these must be able to inhale without injury the vapour of iron, copper, zinc, and others of our familiar metallic elements. For the solar atmosphere, to a depth of a few hundred miles, is loaded with these vapours, all glowing with intensity of heat.

But how, it will be asked, can a fact of this sort become known to astronomers? It seems incredible that an observer on the sun, even if armed with the most powerful instruments used by terrestrial astronomers, could ascertain that the lower strata of our air are loaded with the vapour of water. How then can the terrestrial observer learn that the lower strata of the solar atmosphere are loaded with such and such vapours?

The telescope, as I have said, can teach nothing. But the spectroscope can not only teach us this lesson about the solar atmosphere, but it has been teaching men that lesson for more than half a century, though the lesson has only of late been understood. The sun's light, shining through the complex atmosphere of glowing vapours, is deprived of certain of its rays by the absorbing power of these vapours; and, accordingly, when that light is spread out into the rainbow-tinted streak called the solar spectrum, those rays are found 
to be wanting, the streak being seen to be crossed transversely by multitudes of dark lines. This had, however, been known for many years, and even the general interpretation of the fact had been ascertained for nearly a quarter of a century, before the true nature of the complex atmosphere was understood. Kirchhoff, the eminent German physicist who detected the secret of the solar spectrum, conceived that the sun's corona is the atmosphere which produces the dark-lines. In other words, he supposed an atmosphere some two or three millions of miles in depth to be in question instead of an atmosphere only a few hundred miles deep.

But when the study of the sun's coloured prominences had shown that at a height of forty or fifty thousand miles there is no glowing iron, or copper, or zinc-no atmospheric matter, in fact, which can by any possibility account for the dark lines in the solar spectrum, Kirchhoff's theory was seen to be untenable. It was clear that, if the absorbing vapours lay above the visible surface of the sun at all, they must needs occupy a shallow region, unrecognised hitherto either by the observers of the eclipse or by spectroscopists.

Now Father Secchi announced in 1869 that he had detected traces of just such an atmosphere. For when he examined with his spectroscope the very border of the sun, he found that the dark lines could no longer be seen; as tbough the light of the glowing vapours themselves-which, examined alone, could show bright lines precisely where the solar dark lines appear- 
sufficed exactly to fill up the gaps caused by the absorptive action of those vapours. Secchi reasoned in this way:-If we examine the intensely bright light of the sun shining through these vapours, we see that this light is deprived of certain rays, and so dark lines appear; but if we could examine the light of the vapours themselves we should see that this light is composed of these self-same rays, and so bright lines on a dark background would appear. Now the latter we cannot do on account of the extreme shallowness of the complex atmosphere ; we can, however, by examining the very edge of the sun, obtain light so combining the two kinds that there will neither be dark lines on a bright background nor bright lines on a dark background, but a continuous rainbow-tinted streak produced by the combination of the two.

It seemed to Professor Young, of America, that during the eclipse something more might be achieved. For at the very moment when the moon has just concealed the true solar disc, the light of the shallow atmosphere must be shining alone. The reader can readily illustrate the case as follows:-Let a shilling represent the sun's disc, the narrow raised edge representing the shallow solar atmosphere; and let a halfpenny be passed over the shilling, as the moon's disc passes over the sun's in an eclipse. Then as soon as the advancing edge of the halfpenny has reached the inner boundary of the raised edge, it will be seen that a fine sickle of that raised portion remains in view, the thickest part of the sickle being at the point 
where the halfpenny has just reached the raised edge. At the corresponding moment of a total eclipse a sickle of the sun's complex atmosphere is still illuminating the air around and above the observer ; and an ordinary spectroscope, such as would show the solar dark lines if turned towards the sky in time of sunshine, ought, if turned towards the sky at this moment, to show all those lines as bright lines; while one of those spectroscopes with which astronomers study particular parts of an object, if directed towards the widest part of the fine atmospheric sickle, ought to show the same lines in a much more conspicuous manner.

Professor Young, in December 1870, was able to show that both these methods can be employed; for he himself used the latter, having his telescope directed (and kept directed by clock-work) towards the point where the moon would obliterate the last fine edge of direct sunlight. And he prepared an ordinary telescope for the use of Mr. Pye (a young English gentleman residing near the place where Professor Young's party were stationed), and instructed Mr. Pye what to look for. Both observers saw that as the rainbow-tinted streak forming the solar spectrum faded away at the instant of totality, there sprang into view a myriad-lined spectrum - the spectrum, in fact, of the sun's true atmosphere, now for the first time recognised.

During the recent eclipse, Colonel Tennant, Captain Maclean, and several other observers, saw the beautiful bright line spectrum of the sun's glowing atmo- 
sphere. One or two observers failed to do so; but it need hardly be said that these failures prove nothing except the extreme delicacy of the observation. The positive results, which need alone be considered, prove decisively that next above the sun's light surface there lies an exceedingly complex, but relatively very shallow, atmosphere, loaded with the glowing vapours of all those elements-metallic or otherwise-to which the dark lines of the solar spectrum are known to be due.

Next in order comes the sierra, or red envelope, sometimes called the chromosphere (or more correctly the chromatosphere ${ }^{1}$ ).

The sierra is a far more extensive atmospheric region than the complex atmosphere of Young and Secchi. Its average depth is probably about five thousand miles. Its chief constituent is glowing hydrogen, but it contains other elements, and is indeed far less simple in constitution than was supposed a year or two since. That this is so is proved by the fact that Professor Young has counted no less than one hundred and twenty lines in the spectrum of this red atmosphere.

Above the red sierra, and reaching even beyond the loftiest prominences, lies yet another atmospheric envelope-the inner corona, as it has been called.

The consideration of this important solar envelope leads us to one of the most important of the dis-

' Strictly speaking, the word 'chromosphere' is as incorrect as 'phography' would be for photography, 'chromic' for chromatic, or 'chronatic' for chronic. 
coveries made during the late eclipse. It had long been recognised that the solar corona appears to consist of two portions distinct from each other. The inner portion received (from the Astronomer-Royal, we believe) the name of the ring-formed corona, because not marked by any noteworthy indentations, gaps, rifts, or the like, but presenting the appearance of a somewhat uniform ring of whitish light around the black disc of the eclipsing moon. It was to this corona that some of the observers of the eclipse of December 1870, mistakenly supposing its recognition at that time to be a real discovery, proposed to assign the name leukosphere. The term was intended to indicate the apparent whiteness of the inner corona. But under favourable circumstances the envelope presents a slightly ruddy tinge, with traces of green.

Astronomers had begun to recognise the fact that the inner ring-formed corona must be a solar appendage, whatever may be thought of the fainter radiated corona which surrounds it. The light of the ringformed corona had been examined with the spectroscope, and appears to resemble in some respects that of the aurora borealis, insomuch that some astronomers expressed their belief that this envelope is a perpetual solar aurora. The startling nature of this conception will be realised when it is mentioned that at a moderate computation the ring-formed corona has a depth exceeding twenty times the diameter of the earth on which we live, while the actual portion of space occupied by these auroral lights (if the theory 


\section{2 LIGHT SCIENCE FOR LEISURE HOURS.}

be true) must exceed the volume of the earth more than fifty thousand times. Besides such displays as these, the most glorious auroras that have ever illuminated terrestrial skies sink into utter nothingness.

But some difficulty was experienced in demonstrating that the spectrum on which these ideas had been based belonged in reality to the ring-formed corona. The study of the sun's surroundings by spectroscopic analysis is not free from certain causes of perplexity. To show how these may arise, we need only consider a case which anyone possessing a small spectroscope (one of Browning's miniature spectroscopes, for instance) can readily test for himself. If such a spectroscope be turned (with suitable precautions) towards the sun, we see the principal solar dark lines, and we know that those lines teach how the sun's light is partially absorbed by the vapours of certain elements existing in his atmosphere. But if next we direct the instrument towards the sky, we see precisely the same spectrum, only reduced in splendour. Yet the vapours of iron, copper, zinc, and so on, do not exist in the sky. The fact really is that we receive from the sky reflected sunlight, and therefore we can trace in the spectrum of skylight the dark lines belonging to sunlight. And in exactly the same way, the sky during total eclipse, though not very brilliantly illuminated, is nevertheless lit up to some extent by the corona prominences, and chromatosphere, and therefore the skylight must supply, however faintly, those bright lines which belong 
to the spectrum of the gaseous solar surroundings. How is the observer to tell, when he obtains these bright lines from any given part of the corona, that they actually belong to that part of the corona and not to the light of the sky?

Now Professor Young, in December 1870, dealt with this difficulty in a very subtle and masterly manner. I have already mentioned two different ways in which spectroscopic analysis can be applied. In one we are analyzing the light from a considerable range of space, in the other we study only that light which comes from a certain definite direction. Professor Young, who had applied both methods to the shallow complex atmosphere, applied both, with similar success, to the inner corona. Let us suppose that by the former method the whole of the region of sky occupied by the inner corona was supplying light for the spectroscope to analyze; and that by the latter only a fine linear strip from the brighter part of the inner corona was being analyzed. Then clearly and without entering into niceties of detail, if the bright line spectrum we are considering belongs in reality to the inner corona, we should find the true coronal lines relatively much brighter by the former method than by the latter. For in the former there is the great extent of the inner corona to compensate the feebleness of its inherent luminosity, in the latter there is no such compensation.

Carefully studying the relative brightness of the suspected coronal lines, when the two methods of III. 
observation were applied, Young inferred that a certain green line belongs unquestionably to a region of luminous matter not less extensive than the inner corona. It appeared tolerably safe to conclude that the inner corona was the actual source of his peculiar light. And if the resemblance between this light and that of the aurora borealis were admitted, it appeared reasonable to infer that the inner corona is a perpetual solar aurora, as had been suggested in 1869 .

But although the reasoning of Professor Young was so nearly conclusive, that he must be regarded as in effect the discoverer of the important facts just mentioned, yet it seemed desirable to astronomers to endeavour to obtain even more convincing evidence. We have seen the difficulty which lay in their way ; it remains to be shown how, during the recent eclipse, that difficulty was overcome.

They had hitherto dealt with the spectral line or lines of the inner corona. Those lines are in reality coloured images of the slit through which the spectroscopist admits the light which he proposes to examine; and therefore their shape can teach him nothing about the source of light, their position (or which is the same thing, ${ }^{1}$ their colour) being all that the spectroscopist considers. But suppose he uses no slit, then instead of a series of images of a slit he will have a series of

1 The same thing, because a bright line corresponding to any position along the rainbow-tinted spectrum has the colour proper to that position. In fact, spectroscopists indicate the position of a bright line by reference to its colour-saying a line in the red, or in the blue green, or the like. 
images of the source of light. If the source of light is the sun or any object shining with all the colours, the different images will overlap and he will see simply 'Newton's spectrum,' a rainbow-tinted streak of extreme beauty and splendour, but nevertheless what the spectroscopist describes as an 'impure spectrum,' because in it a multitude of overlapping images are present. If, however, the source of light emits rays of certain colours only, then there will be separate images of these colours, each clearly discernible in all its details. For example, let us suppose that in a little conical flame of great heating power but small luminosity, a chemist places a small quantity of sodium and lithium. Then when he looks at the flame through a spectroscope without using a slit he will see a little conical yellow flame, and close by it a little conical and rather faint orange flame, and further away a little conical red flame; whereas if he had had a fine slit to his spectroscope he would have seen three fine lines, a yellow one due to the sodium, and two lines, one orange and the other red, due to the lithium.

Now if the reader has followed this brief but necessary explanation, he will see that the astronomer possessed the means of at once solving the difficulty of the corona. So long as he used a slit he obtained a bright green line which might not come trom the corona, but from the illuminated sky in the same direction; but if he removed the slit and then saw a green image of the corona, he would no longer be in doubt. For the illumination of the sky could not form 
an image of the corona, any more than the sky we see in the daytime forms images of the sun though shining with solar light. If the observer examining the corona with a suitable spectroscope not provided with a slit saw a green image of the corona, it could only be because the green light came from those parts of the sky where the corona was actually seen, and from no other parts.

Now this experiment was precisely what Respighi, the eminent Italian astronomer, determined to attempt. He had an instrument (made for him in 1868) which seemed to him admirably adapted for the purpose; and accordingly he took this instrument with him to India, and, stationed at Poodocottah, he successfully applied it to the solution of the problem which had so long perplexed astronomers. His observations involved results of interest, relating to the coloured prominences, since these as well as the inner corona were presented in spectrally shifted images.

'At the very instant of totality,' he says, 'the field of the telescope exhibited a most astonishing spectacle. The chromatosphere at the edge which was the last to be eclipsed, surmounted by two groups of prominences, one on the right, the other on the left of the point of contact, was reproduced in four spectral colours, with extraordinary intensity of light, and the most surprising contrast of the brightest colours, so that the four spectral images could be directly compared and their minutest differences easily made out. All these images were well defined, and projected in certain coloured 
zones, with the tints of the chromatic images of the corona. My attention was mainly directed to the forms of the prominences, and I was able to determine that the fundamental form, the skeleton or trunk, and the principal branches, were faithfully reproduced or indicated in all the images, their extent being, however, greatest in the red, and diminishing successively in the other colours down to the indigo images, in which the trunk alone was reproduced. In none of the prominepees thus compared was I able to distinguish in the yellow image parts or branches not contained in the red image. ${ }^{1}$ Meanwhile the coloured images of the corona became continually more strongly marked, one in the red corresponding with the red line of hydrogen, another in the green' (corresponding with Professor Young's green line), 'and a third in the blue, probably corresponding with the blue line of hydrogen.'

1 The interest of the question whether such differences would be perceived resides in the fact that the red, green, and indigo images are all due to hydrogen, but the yellow to another element, present in the prominences; and Respighi hoped to ascertain whether this element extended beyond, or not so far, as the hydrogen. For our own part, we are disposed to place rery litttle reliance on some of the facts observed in this particular part of Respighi's work. With the red and yellow images shining in full splendour, he would naturally be unable to see the fainter parts of the indigo images; but these darker images are probably at least as extensive as they are certainly as well-defined as the others. For Secchi, in studying the prominences by the spectroscopic method, selects the indigo images for the purpose, because he has found that cateris paribus the indigo images appear the most complete. In Respighi's work other things were not equal. Similar remarks apply to the apparently inferior extension of the blue green image of the inner corona. 


\section{I8 LIGHT SCIENCE FOR LEISURE HOURS.}

Thus not only has the fact been proved that the light producing the 'green line comes, as Young had reasoned, from the inner corona, but also that this corona consists in part of glowing hydrogen. And when I say 'in part,' I do not mean that throughout a portion of its extent the corona consists of hydrogen; but that one of the elements of which the corona is formed is the familiar gas hydrogen. It appears from the sequent remarks of Professor Respighi that the hydrogen extends as far, or very nearly so, as the matter, whatever it may be, which produces the green light of the corona. Before quoting his words, I remind my readers that what: Respighi saw was three pictures of the corona in three different places-one picture produced by the red part of the corona's inherent luminosity, another by the green part, and another by the blue part of that luminosity. The three zones he speaks of are not three distinct envelopes, but three pictures of one and the same element. Just as the spectroscopist in the case of our imagined experiment with the lamp-flame could not infer that there were three small conical flames, because he saw three images of the single small conical flame, so Respighi knew that the three rings of light which his telescope (spectroscopically armed) presented to his view, were spectral images of one and the same object, the inner ringformed solar corona.

'The green zone surrounding the disc of the moon,' he says, 'was the brightest, the most uniform, and the best defined. The red zone was also very distinct and 
well defined; while the blue zone was faint and indistinct. The green zone was well defined at the summit, though less bright than at the base; its form was sensibly circular and its height about six or seven minutes' (corresponding to a real depth of from 160,000 to 185,000 miles). 'The red zone exhibited the same form, and approximately the same height as the green; but its light was weaker and less uniform. These zones shone out upon a faintly illuminated ground without any marked trace of colour. If the corona contained rays of any other kind, their intensity must have been so feeble that they were merged in the general illumination of the field.'

' Soon after the middle of the totality,' he proceeds, ' there appeared on the sun's eastern edge a fine group of prominences formed of jets, rather low, but very bright, some rectilinear, others curved round the sun's limb, and exhibiting the intricate deviations and all the characters of prominences in the neighbourhood of solar spots. The brightness and colour of these jets were so vivid as to give them the appearance of fireworks. The spaces between some of the jets were perfectly dark, so that the red zone of the corona appeared to be entirely wanting there.' (It will be remembered that the red images of the prominences, and the red image of the corona, were necessarily accordant in position, since they were produced by the same kind of light, the red hydrogen rays.) 'Perhaps, however, this was only the effect of contrast due to the extraordinary brightness of the neighbouring jets. I 
have thought it right, however, to refer to the peculiarity because the appearance of interstices or double spaces, between prominences of considerable brightness, is often observed by means of the spectroscope independently of total eclipses. The green and red zones of the corona were well developed on the western as well as on the eastern edge of the sun, while the blue remained faint and ill defined.'

It seems tolerably clear that Respighi saw, in the green image, the full extension of the inner corona; for the edge of that image was well defined, as it would certainly not have been if the observed extension had depended only on the observer's power of recognising faint luminosity. In the latter case there would have been a gradual fading off, precisely as in the case of the blue image. It is important to notice this point; because Mr. Lockyer (probably observing under less favourable conditions) could only trace the green image of the inner corona to a height of about two minutes, or less than one-third of the height observed by Respighi; and we might be led to infer that as Respighi saw the green coronal image extending so much farther from the sun than as observed by Lockyer, so under yet more favourable circumstances the image might have appeared higher still. The well-defined outline recognised by Respighi renders this inference inadmissible; and we may in fact regard the extension of the inner corona as definitely determined by his observations. On the other hand, the relatively small extension of the blue image does not necessarily prove 
that the blue light does not emanate from the whole of the inner corona, since the ill-defined nature of the image affords reason for believing that its observed extension was merely a question of eyesight.

We have then-and the result cannot but be regarded as one of the most important ever established during eclipses-the conclusion that surrounding the sun to a depth of nearly two hundred thousand miles, there is an envelope of hydrogen mixed with an element capable of emitting the green light so often referred to in the above description.

But I am led to pause here, in order to inquire what element it is which supplies the green light. Now here we have a most interesting question to consider. For the light of our own auroras shows this very green line. Professor Young has tested the matter in a way which prevents all possibility of doubt. Using a spectroscope of almost unmatched power, he could recognise no difference of position between the green line of the aurora, the green line of the inner corona, and a green line seen always in the spectrum of iron. But of all elements in the universe iron seems to be precisely the element which ought not to be present, either in the regions whence comes the light of our auroras, or in the inner corona of the sun. Iron in the solid state might indeed be present from time to time in the upper regions of our air, because iron is. nearly always present in meteorites, and meteorites are always passing through the upper regions of the air in greater or less numbers. But the green line, if it in 
truth appertains to the iron spectrum, implies the existence of the glowing vapour of iron; and heat of great intensity is required to vaporise iron. It is, however, possible that electrical discharges may be in question. We know, indeed, that the aurora is an electrical phenomenon, although we do not as yet know exactly how the electrical action is caused, or what its nature may be. We should certainly find many difficulties obviated if we extended the same explanation to the solar corona, since many of the phenomena which it presents are strikingly suggestive of electrical action. Viewing the green light in this way, and not venturing at present to determine the precise manner in which electrical action is excited, we should be led to recognise the presence of iron in the corona, the iron not being in the state of vapour, but giving the vapour spectrum of iron on account of the electrical discharges continually taking place between the particles of solid or liquid iron. It might even be that the hydrogen lines from the corona may be referred to electrical action, and not to the actual heat of the hydrogen present throughout the inner corona. In this way we may obviate a difficulty referred to above when the sierra was described. We may regard the sierra as the region where the sun's hydrogen atmosphere actually glows with the intensity of its own heat; and the inner corona as the region where the same atmosphere is traversed by continual electrical discharges, which cause the bright lines of the hydrogen to be recognised by our spectroscopists, though not with the same 
brightness as from the region of actually glowing hydrogen. ${ }^{1}$

A difficulty remains in the fact that the spectrum of iron contains upwards of four hundred and fifty bright lines, and that the green line in question is not even the most conspicuous of these. Nor, indeed, is it absolutely certain that this particular line, though always seen in the spectrum of iron, belongs actually to that metal. At present, however, the most probable conclusion appears to be that which has been presented above; and we may suppose either that the other lines of iron are really present, but too faint for recognition, or that their absence is due to the special circumstances under which iron exists in the upper regions of our own air and in the rare hydrogen atmosphere of the sun.

In our journey outwards from the sun's light surface, we have now approached the inner boundary of the most interesting of all the solar surroundings, the outer radiated corona, the reality of which had been so long disputed. Respecting this appendage-occupying a space enormously greater than any structure known

1 It should be explained that if an electrical discharge passes from. iron to iron through hydrogen, the observed spectrum is a combination of the iron spectrum and the hydrogen spectrum. Now the actual brightness of light in this case is not inferior to that of hydrogen glowing with intensity of heat; but the total quantity of light is less than that which would be obtained if the whole of the hydrogen in the tube were so glowing. In like manner, the supposed electrical discharges in the sun's hydrogen atmosphere would produce a light as intense in itself as that of the sierra; but as the discharges would cause portions only of the inner corona to flow with this light, the total luminosity would be far inferior to the luminosity of the sierra, where all the hydrogen is aglow with its own heat. 


\section{LIGHT SCIENCE FOR LEISURE HOURS.}

to astronomers-the recent eclipse observations have supplied most interesting information.

Let us in the first place consider the actual appearance of this object as seen under the favourable circumstances of the late eclipse. The following description is taken from a series of letters which appeared in the columns of the Daily News. 'There in the leaden-coloured utterly cloudless sky,' he writes, 'shone out the eclipsed sun! a worthy sight for gods and men. There, rigid in the heavens, was what struck everybody as a decoration-one that emperors might fight fora thousand times more beautiful than the Star of India (even where we are now)-a picture of surpassing loveliness, and giving one the idea of serenity among all the activity that was going on below; shining with a sheen as of silver essence, built up of rays almost symmetrically arranged round a bright ring, above and below with a marked absence of them right and left, the rays being composed of sharp radial lines, separated by furrows of markedly less brilliancy.'

It is very interesting to notice the greater extension of the corona above and below. For at Bekul, where the observations were made, the sun was close to the horizon, and his equatorial zone was nearly upright or vertical, so that the observation shows that the extension of the radiated corona is greatest opposite the sun's equatorial regions. It is worthy of notice that Father Secchi had considered this fact to be apparent in the photographs which he obtained during the eclipse of the sun in 1860 . 
Let us next consider the work of a practised draughtsman, Mr. Holiday. 'This gentleman,' says the Daily News correspondent, 'proposed to sketch with his right eye while the left was at the eye-piece of the telescope; and, more than this, he did it. ... On the appearance of Baily's beads' (that is, at the moment when the last fine sickle of direct sunlight broke up into small arcs of light), ' he removed the dark glass from the eye-piece of his telescope, but replaced it instantly, finding that even the feeble light was still too strong for the eye that had firmly resolved to note each delicate feature of the corona. Still, the time was not lost, for in that momentary glance he saw doubtless what have been called the "rays before totality," which he at once recognised as the two great lines which marked the limits of the advancing shadow. After a few seconds the glass was removed, and there in all its glorious beauty was a grand corona of the most fantastic type, not unlike the one given by Iiais. To outline this was the work of a few seconds. Curiously enough, there are points of difference and points of agreement between this drawing and the photographs, which will, doubtless, when the time comes, nndergo the most searching examination. After the middle of the eclipse another drawing was made, showing that the corona had become much more diffuse than at its first appearance, and maintained the same form nearly till the re-appearance of the sun.'

It is to be noted, as respects this account, that the 
picture by Liais of the corona as he saw it during the eclipse of 1858, is one which has been ridiculed as altogether a work of the imagination. It presents the corona with peculiarities of detail so remarkable, that if we regard them as real, they dispose finally of the theory that the outer corona is merely due to the illumination of our own atmosphere. Accordingly, the advocates of the atmospheric theory had scouted the pretensions of Liais's picture; and even many who regarded the corona as a solar appendage, could scarcely believe that some of the strange figures shown in the picture were not to some extent idealized. Here, however, we have such figures reproduced by a gentleman whose skill in draughtsmanship will hardly be questioned, while the fact that Mr. Holiday has not advocated any theory of the corona, removes all possibility of doubt as to the accuracy of the drawing being unconsciously affected by preconceived opinions on the part of the draughtsman. I venture to take exception to the remark that the corona had become more diffuse by the middle of the totality; for, it is to be remembered that, with the progress of totality, the observer's power of appreciating faint light would naturally increase, and that accordingly he would be enabled to recognise those outer and fainter parts of the corona which had in the first instance escaped his notice,

The consideration of circumstances such as these causes me to attach so much the more value to the photographic records of the eclipse, which are not 
liable to be affected by physiological peculiarities. From the moment when totality began, the photographic plates were set one after another to record the aspect of the corona, without any fear that the plates exposed earlier or later would be more or less sensitive to the influence of the corona's very delicate light. As yet the results have not been available for examination in Europe; but we have from all sides the announcement that the photographs represent the corona as unchanged in form throughout the totality, with persistent rifts, extending to a great distance from the sun. This is, in effect, decisive. There was room for a shadow of doubt (at least in some minds) when, in December 1870, Mr. Brothers obtained, in the last eleven seconds of totality, a picture showing wellmarked rifts in an extensive corona, ${ }^{1}$ for there were no sufficient means of proving that the same rifts existed at the beginning of the totality. But now all doubts of that sort are finally disposed of ; and since radial beams in our own atmosphere, or produced by the passage of the sun's light past the irregularities of the lunar surface, must inevitably have changed markedly in position during the progress of totality, we have decisive evidence against the two theories which had

1 Mr. Brothers's picture showed the corona widest on the west, whereas a picture by Lord Lindsay seemed to show the corona widest on the east; and great importance was attached to the circumstance. But on a careful examinasion of the prominences shown in the two pictures, it became clear that one of the pictures had been by some accident inverted. So soon as the pictures were so placed that the prominences were brought into agrcement, the corona was found to extend towards the same side in each. 
been urged against the existence of the outer solar corona as an objective and circumsolar reality.

But the recent eclipse has also supplied instructive evidence respecting the nature of the outer radiated corona; for the spectroscope has been applied by one of the most skilful astronomers of the day to the analysis of the coronal light.

Let us first see what Mr. Janssen, the astronomer in question, has to say respecting the appearance of the corona. His remarks on this point are not wanting in definiteness; and they are particularly valuable because he observed the corona from a station raised far above those denser atmospheric strata which are most effective in concealing the more delicate details of the coronal structure. 'I have mounted the central ridge of the Neilgherries,' he wrote, ' which has summits of nine thousand feet in height, and whence, according as we turn to east or west of the ridge, we see the Carnatic plains on the Coromandel Coast, or the plateau of Mysore, as far as the Ghauts.' At this fine station, Janssen was favoured with weather of exceptional clearness; and altogether it is probable that never since eclipse observations began, had the corona been studied under such favourable circumstances. In the following sentences Janssen presents the results of his general observations. 'Nothing could be more beautiful or more luminous; with special forms excluding all possibility of a terrestrial origin. The result of my observations at Sholoor,' he says, 'indicates without any doubt the solar origin of 
the corona, and the existence of substances beyond the chromatosphere. I think the question whether the corona is due to the terrestrial atmosphere is disposed of (tranchee), and we now have before us the prospect of the study of the extra-solar regions, which will be most interesting and fruitful.'

In the spectroscopic study of the corona Janssen achieved a noteworthy success. Hitherto astronomers had failed in recognising on the faint rainbow-tinted spectrum, forming a background, as it were, to the distinctive bright-line spectrum of the corona, those dark lines which are seen in the spectrum of solar light. The inference was that very little or none of the coronal light is reflected sunlight. Janssen, however, besides detecting several bright lines which had not hitherto been recognised, saw also the chief solar dark lines. Strangely enough, he appears to infer from their presence that the corona exercises an absorptive effect on light which would otherwise produce a rainbow-tinted spectrum unstreaked by dark lines. To me, the more natural explanation appears to be that a portion of the coronal light is due simply to the reflection of sunlight from the cosmical matter undoubtedly surrounding the sun. Janssen himself recognises the existence of such matter, since in his remarks on his observations he says, 'Besides the cosmical matter independent of the sun, which must exist in his neighbourhood, the observations demonstrate the existence of an excessively rare atmosphere, mainly of hydrogen, extending far beyond the III. 


\section{I3O LIGHT SCIENCE FOR LEISURE HOURS.}

chromatosphere and prominences, and deriving its supplies from the very matter of the latter, matter projected (as we daily witness) with such extreme violence.'

Here, properly speaking, my remarks respecting the recent observations should be drawn to a close. We have seen how those observations throw a new light on all the circumsolar appendages, from the complex shallow envelope forming the true solar atmosphere, to the outermost extensions of the radiated corona. The eclipse revealed nothing, directly, respecting matter outside the coronal radiations. But indirectly it gave important evidence respecting a solar appendage which attains a far greater extension. I refer to that strange object, the zodiacal light, emitted by a region which surrounds the sun on all sides, to distances exceeding the orbit-ranges of the planets Mercury and Venus, even if this region do not reach far beyond the orbit of our own earth. It happens, by a strange chance, that the astronomer Liais, whose long-doubted observations of the corona have just been so strikingly confirmed, has but now announced his discovery of the fact that the zodiacal light, when analysed with the spectroscope, gives a faint continuous spectrum. It had been asserted that the zodiacal light gives a spectrum resembling that of the aurora; but grave doubts had been entertained respecting the accuracy of the observations on which this assertion had been based. The observation made by Liais would tend to show that, as had been long 
suspected, the zodiacal light is sunlight reflected from cosmical matter travelling continually around the sun (for we could not expect the solar dark lines to appear in so faint a spectrum). If this is the case, the radiated corona cannot but be regarded as only the innermost part-the core, so to speak- of the zodiacal region. Hence, we should be led to recognise the existence of envelope after envelope around the sun, until even the vast distance at which our earth travels is reached or overpast. We need wonder little that under these circumstances our earth should sympathise with the disturbances affecting, from time to time, the great central luminary of our system, or that she should be thrilled from pole to pole by magnetic tremors, when his orb is excited either by internal throes or by external impulses to intense electrical action.

Good Words: January, 1872.

\section{THE ECLIPSE OF 1878.}

FIrST, let us consider the news respecting the sun's complex atmosphere, extending some 300 or 400 miles from his surface, and containing all or most of those vapours, metallic and otherwise, whose absorptive action produces the dark lines in the solar spectrum. All these lines were seen, as on former occasions, reversed when totality began and ended. But on this occasion, for the first time, the observers were able to distinguish 
known lines, and to note differences of brightness. Thus, near B, one of the nine strong lines to which Fraunhofer assigned the first nine letters of the alphabet (and called large $B$ in the telegram to distinguish it from a certain triple line called little b), several very bright lines were seen. Dark lines belonging to the oxygen and nitrogen of our own air have been observed in this part of the sun's spectrum, and it may be that we here have evidence corroborating Dr. H. Draper's recognition of bright oxygen lines in the solar spectrum. 'Bright line $\mathrm{F}$ was seen,' but that being one of the hydrogen lines, its being seen was nothing new; it would have been surprising, indeed, if it had not been seen. The line 1474 Kirchhoff is one of the most interesting of all. It is the chief bright line of the corona's spectrum. It was once thought to be a line of iron, but Professor Young showed that this could not be the case. The presence of this line among the bright reversed lines, and its absence from the dark lines of the solar spectrum, as ordinarily seen, is an interesting discovery, if it has been certainly established. It would imply that the substance to which the line is due-a substance present in large quantities in the corona of 1869,1870 , and 1871-exists ordinarily at a temperature higher than that of the vapours in the sun's atmosphere; for these absorb more light than they emit, which indicates that they are cooler than the sun's surface beneath them, whereas the matter producing the line 1474 Kirchhoff must be as hot as the surface of the sun, 
since it emits at least as much light as it absorbs. I would recommend to the special attention of spectroscopists a re-examination of the solar spectrum in the region of this line; for, as will presently appear, the sun's surroundings are not now in the same condition as they were in 1869,1870 , and 1871 , when special search was made for the 1474 line. The statement that both the $\mathrm{H}$ lines were reversed is important, as showing that the whole range of the visible spectrum was examined for these reversed or bright lines. The $\mathrm{H}$ lines are near the violet end of the spectrum. They belong to calcium, and, as calcium has been recognised at times in the sierra, we can hardly attach special importance to the fact that these lines were seen in the more complex spectrum of the solar atmosphere below the sierra. The observation was probably only mentioned to show that the array of bright lines was traced up to the violet end of the spectrum. Indeed, quite possibly the bright lines near B were only mentioned for a similar reason, B lying at about the same distance from the red end of the spectrum as $\mathbf{H}$ from the violet end.

Turning next to the sierra, or chromatosphere, we learn that on this occasion its height was far less than in 1869-1871. A query is attached to the height, 2,000 miles, mentioned in the telegram ; but probably the doubt related to the number of ciphers only, not to the ' 2 ,' and if so, we need not concern ourselves with it; for unquestionably the sierra was not so high as 20,000 miles, nor so low as 200 miles, the former 


\section{LIGHT SCIENCE FOR LEISURE HOURS.}

being the height of a goodly prominence, the latter too small a height to be measurable at the sun's distance. The height of the sierra in 1871 averaged some 6,000 miles. But it had been noticed that this envelope had been shrinking as the sun spots diminished in number. The eclipse observations confirm those made by the method devised in 1868 for studying the prominences when the sun is not eclipsed. We thus learn definitely that this envelope, consisting of a mixture of hydrogen, helium (the unknown solar element), sodium, and occasionally magnesium, calcium, and other elements, shrinks during the time when the sun is free from spots to about a third of the height which it attains when the sun shows many spots and is, we may infer, most actively disturbed. As the height is in this case a fair measure of the volume, I may say that the sierra has lost two-thirds of the volume it possessed in 1871 ; and again, as that volume exceeded that of the earth more than 13,000 times, it follows that the loss of volume experienced by the sierra since 1871 exceeds some 10,000 times the entire volume of this earth on which we live. It is clear that this implies a very remarkable change in the condition of the sun himself, though fortunately for us the change is not one which affects in any striking or obvious manner his emission of light and heat.

With regard to the prominences, the evidence given by the eclipse accords (as I expected it would) with that obtained by spectroscopists. They have observed that the prominences had become less and 
less in number, and had still more remarkably diminished in brightuess and in the signs which they afforded of eruptive solar action. The eclipse observers saw only one prominence, resembling the 'horn,' which was the most remarkable feature of the eclipse of 1868 . 'This single prominence was 'very dim;' indeed, the observers in Colorado did not see it at all, for though they mention two protuberances, they add that 'there was an entire absence of the pinkish red flames observed on some previous occasions ;' whence we may safely conclude that by protuberances they mean masses of red matter not extending high above the sierra. We thus find an explanation of the circumstance, which had been thought perplexing, that during some eclipses very carefully observed before the actual recognition of the red prominences no red flames were noticed. We now see that during an eclipse observed when the sun is without spots the prominences might very easily escape recognition, at least by observers not aware of the existence of such objects.

But it was about the corona that astronomers chiefly expected to obtain information during this eclipse, and certainly the result has confirmed their anticipations. First, it was discovered that the corona had changed entirely in form, in size, and in the quality of its light, if not in its physical constitution, since the eclipse of 1871. As observed on that occasion, it extended to a distance of more than 1,000,000 miles from the sun. As photographed, it showed an extension of at least 700,000 miles. It was exceedingly complicated in 
structure. Multitudinous streaks, some few straight, but most showing curvature more or less complex in character, extended from the edge of the sun, and gave to the corona the appearance of luminous hair waved wildly by the wind. (It will, of course, not be supposed that the coronal streamers were actually moving.) Again, the spectroscope showed that a portion of the coronal substance shone with special colours, indicating its partial gaseity. The interior and brighter portion, to a height of nearly 300,000 miles, shone visibly with three of the four tints belonging to glowing hydrogen, and, doubtless, actually with the fourth, though it could not be recognised. Messrs. Lockyer and Janssen, on that occasion, following a method of observation suggested by Professor Young in 1870, saw not the three lines (only) of these colours, but the three coloured images of the corona. They dispensed with the slit, which allows only a fine thread of light to be examined, and simply looked at the corona through the battery of prisms. The dispersive action of the prisms divided the single image seen directly into three, showing a red image in one direction, a blue image in another, and between these, nearer to the latter, a green image. Lastly, in 1871, the corona was not very bright. But on July 29 the corona had shrunk greatly in dimensions, and had increased correspondingly in brightness. It extended to ordinary telescopic vision only about 70,000 miles from the sun's surface, which is considerably less than the height of many prominences which have been seen on former 
occasions. Its brightness was estimated by $\mathrm{Mr}$. Lockyer as exceeding tenfold that of the corona seen in 1871. It was comparatively simple in structure, showing only in two places indications of definite structure close to the sun. Several long rays were seen, how- ever, and I shall look forward with extreme interest to the evidence obtained respecting these features. ${ }^{1}$ For it appears that the doubts which I had supposed 'no longer entertained' respecting the corona, though dismissed of necessity as regards all the complicated details photographed in 1871, still linger as regards the long straight rays, which did not appear on that occasion and naturally were not photographed. The resemblance between these rays and those seen in Secchi's ' hole in a shutter' experiment leads some to imagine that, like the latter, the former are produced by the sun's light shining on matter nearer to the eye than the interposed dark object-the irregular 'stopper' in Secchi's experiment, the moon in the case of a total eclipse. In reality the rays cannot be thus explained, unless we assume that our atmosphere or some other matter capable of reflecting light extends as far as the moon, which few will consider probable. I believe that the observations made on this occasion, and especially the photographs of the corona, will dispose definitely of these last remaining doubts respecting

1 These were observed by Professor Cleveland Abbe extending to a distance of at least five millions of miles from the eclipsed sun, and from their appearance and changes of appearance it was clear that they were due to meteoric streams near the sun. See further the essay on 'The Earth in Meteoric Shadow' which follows. 
the solar corona. The occasion was a very favourable one for the purpose, many of the stations being high above the sea level. While the corona as a whole had thus shrunk in extent and increased in brightness, losing also the complexity of structure it had shown in 1871, it had changed still more significantly in another way. Dr. H. Draper and Mr. Lockyer repeated the observation which the latter had made in 1871 , but they saw no coloured images of the inner corona, and the spectroscopists saw no bright lines in the spectrum of the corona. In fact, the corona no longer showed those signs of being partly gaseous which had been recognised in 1869 , 1870, and 1871. Not only so, but the rainbow-tinted background corresponding to the non-gaseous part of the corona showed no dark lines. It was not, therefore, reflected sunlight only or chiefly, as it had been in 1871, when Janssen recognised the solar dark lines in this part of the corona's light. It was, in fact, the light which comes from glowing solid or liquid matter. The inference seems clear that now, when the sun is without spots, the corona, greatly shrunk in size, and, as it were, drawn inwards towards the sun, is so intensely heated as to be self-luminous - a result not very surprising when we remember that the heat, even at its outermost part, is 100,000 times that of vertical sunlight on the earth. Singularly enough, on this occasion, when first decisive spectroscopic evidence was obtained respecting the intense heat of the minute particles probably forming the corona, the heat received from the corona was actually measured. The ingenious 
Edison, who has recently added to his feat of crystal. lising sound (to speak fancifully) the seemingly not less impossible achievement of ' talking a hole through a deal board' (to speak strictly by the letter), has devised a most ingenious instrument, which he calls "the tasimeter (or strain-measurer), for estimating small changes of temperature. This instrument was employed during the recent eclipse to compare the heat received from parts of the sky remote from the eclipsed sun with that received from the corona. It gave the clearest possible evidence of the fact that heat comes to us as well as light from this vast solar appendage.

The last discovery to be noticed (as bearing at least on questions of solar physics) is in some respects even more important than any we have yet considered. Every student of astronomy knows that after sunset in spring, and before sunrise in autumn, a faint illumination of the sky can be seen above that part of the horizon beneath which the sun lies. It has long been recognised that the lens-shaped light thus seen, called the zodiacal light, indicates the presence of matter surrounding the sun, to a distance exceeding probably that of Mars, and certainly that of Venus, from the sun. It had been suggested that the inner and brighter portion of this solar appendage might be recognised during total eclipse by an observer who should devote himself specially to the task. In the monthly notices of the Astronomical Society for 1869-70, page 149, the present writer, in a paper on 'The Solar Corona and the Zodiacal Light,' pointed out the value of observations 
made at a distance of several degrees from the sun, a ' dark disc' being so arranged as to conceal ' a circular space extending a degree or so beyond the eclipsed sun' all round. During the eclipse of 1870, in anticipation of which this suggestion was made, Professor Newcomb endeavoured to observe the zodiacal light in this way. But he was not successful; in fact, the sky was hazy and the circumstances in other respects unfavourable for an observation of the kind. On the present occasion, however, he has applied the method successfully. ' 'Professor Newcomb,' we read, ' who had erected a screen on a high pole, thinks he detected the zodiacal light extending six degrees from the sun.' The telegram containing this passage comes from an observer who would be likely to place very little faith in an observation of the zodiacal light during total eclipse, as he had, in fact, adopted the opinion that the zodiacal light is a terrestrial phenomenon. Professor Newcomb would be the last in the world, I think, to describe an observation in terms so vague, and we should not be at all surprised to learn that he was quite satisfied with his recognition of the zodiacal light. The observation is one which will probably be renewed on future occasions. It may turn out that the zodiacal light, like the corona, varies with the varying condition of the sun as to spots and other signs of disturbance. 


\section{THE EARTH IN METEORIC SHADOW.}

THE occurrence of certain spells of exceptionally cold weather in February, April, and May has long been recognised as among the most mysterious of meteoro logical phenomena. Not in every year, but still so often that the change is recognised by other than scientific observers, the temperature falls from about the 7 th to the 12th of February, from about the 10th to the 14th of April, and from about the 10th to the 14th of May. It had been thought sufficiently strange that this should have been noted, as Kaemtz long since pointed out, throughout the whole of Europe; and Erman had been led, by this evidence alone, to ascribe the peculiarity to some extra-terrestrial cause. When it was found that the peculiarity is observable in North America also, the evidence in favour of some cosmical cause for the phenomena was greatly strengthened. Still there were those, myself among the number, who could not accept the only extra-terrestrial cause which had been assigned, and were disposed to believe that possibly some process usually or ordinarily taking place at the observed times, as, for instance, the breaking up of special ice-fields in February, April, and May, might occasion the lowering of the temperature observed at these seasons. Now, however, evidence has been obtained which seems to show that the cooling in question affects not the northern hemisphere alone but 
the whole earth, insomuch that the belief seems forced upon us that the cause, whatever it may be, is to be sought outside the earth. And just now, when this explanation of a meteorological phenomenon is suggested, astronomical evidence comes in which seems to show how the lowering of the earth's temperature may be explained-that Adolph Erman, in point of fact, though wrong as regards nearly all the details of his theory, was not so far wrong as regards the general theory as had been supposed by some of his critics.

Let us, in the first place, consider the evidence as to the existence of these cold spells.

Theoretically, there should be each year a gradual rise of temperature from about the middle of January to about the middle of July. At a first view it might seem that the winter and summer solstice should be the time of least and greatest heat; but as a matter of observation the greatest heat is never attained in the case of any body or collection of bodies exposed to the action of a source of varying heat, at the time when the heat emitted from that source has its maximum amount, nor, vice versâ, the greatest cold when the emitted heat is least, but always somewhat later. Each day, for instance (in steady weather), the greatest warmth is attained at about two in the afternoon, while two in the morning is the time of greatest cold, on the average, throughout the diurnal hours.

Now we might fairly expect that if the average daily temperature for a great number of years at any given place were observed, the variation of temperature 
would be found tolerably uniform. Thus for London and its neighbourhood we might expect to find something like that variation of temperature which is indicated in our almanacs (which leave altogether out of account, for some unknown reason, the anomalies we are considering). So that if we represented the average temperature for successive days by an upright line, drawn from a horizontal line indicating the positions of successive days in the year-or, to speak technically, if we represented daily average temperatures by a series of ordinates to a line along which time was indicated by abscissas-we might expect the curve passing through the upper extremities of the uprights to have a wave-like form, the crest of the wave lying above the part of the horizontal line corresponding to the middle of July, while the hollow or valley of the wave lay above the part corresponding to the middle of January.

But this is not found to be the case. I have before me as I write a diagram drawn by myself several years ago in illustration of my article on the Climate of Great Britain in the second series of my 'Light Science for Leisure Hours.' I drew a large rectangle, and divided each of its longer sides into 365 parts, to represent the days of the year, and drew through the points of division a series of 365 uprights, on which I marked the mean annual temperatures for the corresponding days, the mean having been derived from Greenwich observations ranging over forty-three years. A connected line was carried then through the 365 extremities of these lines. 
The resulting curve is remarkable in many respects. The lowest point occurs in the first half of January (with a singular, though slight, rise of temperature on about the 10th or 11th), between two equal depressions a few days before and after. From the middle of January there is a rapid rise to the beginning of the last week, when there is a sudden sharp fall of temperature to the beginning of February. Then comes a fall which causes one of the most marked depressions in the whole curve, though strangely enough, when a curve is run through the alternations in January and February so as to leave as much space above as below, the mean temperature for February is found to be above rather than below the average. Still it remains the case that the most marked interruption of the upward rise occurs in February; the greatest depression corresponding to the time from February 10th to February 14th. From February to the beginning of April the rise is tolerably uniform, but from April 10th to the 14th the curve which had been rising rather sharply descends as sharply, and then ascends again, so that this part of the curve is like the letter $s$ placed thus $\omega$. Again, the rise is steady or nearly so till the beginning of May, but from the 9 th to the 14th of May we have a depression almost exactly like that observed in the second week of April. Thence the rise to about June 29 is uniform, though wave-like, i.e., the slope is not constant, but, as we might expect, diminishes as the crest of the wave is approached. In the first week of July the curve is marked by two or 
three small undulations, which lie nearly in a horizontal direction, instead of indicating the continued rise we might fairly expect at this season. In the last half of July there is a rise to the highest part of the curve, which then begins to fall rather sharply, the fall being less rapid after August 10 (instead of more rapid as we might fairly expect). Thence, to the last half of November, there is a tolerably steady fall, but then occurs a rise, making the last two or three days of November and the first week of December considerably warmer than they theoretically should be. After the middle of December there is a very rapid fall in the temperature curve, but a rise occurs in the last week of December somewhat similar to that observed in the last week of November.

The most marked features of the curve are the three cold periods of February, April, and May, and the warm period at the beginning of December.

Let us turn, however, from the south to the north of Great Britain, and see whether the peculiarities noted at Greenwich exist at Edinburgh.

Buchan, in his Handy Book of Meteorology, remarks that the results of all observations hitherto made are unanimous in showing that ' there are certain periods, more or less defined, when the temperature, instead of rising, remains stationary, or retrogradesinstead of falling, stops in its downward course, or rises-and at other times falls, or rises for a few days, at an accelerated speed. I have examined,' he says, ' the temperature of Scotland, for a number of years, III. 
and have shown that the following interruptions occur from year to year, with very rare exceptions :-

$$
\begin{aligned}
& \text { Six cold periods . }\left\{\begin{array}{l}
\text { 1. } 7 \text { th to 10th February. } \\
\text { 2. 11th to 14th April. } \\
3.9 \text { th to 14th May. } \\
4.29 \text { th June to 4th July. } \\
5.6 \text { th to 11th August. } \\
6.6 \text { th to } 12 \text { th November. }
\end{array}\right. \\
& \text { Three warm periods } \cdot\left\{\begin{array}{l}
\text { 1. } 12 \text { th to } 15 \text { th July. } \\
\text { 2. } 12 \text { th to } 15 \text { th August. } \\
3.3 \text { rd to } 9 \text { th December. }
\end{array}\right.
\end{aligned}
$$

It will be seen that the six most remarkable cold periods, and the chief of the warm periods, are recognisable in Scotland as well as in England.

But on the Continent, also, these anomalies have been clearly recognised. The cold weather which occurs in May is prominent in the weather saws of every country in Europe. Mädler examined the mean temperatures for May, as determined from the Berlin observations for eighty-six years (Verhandlung des Vereins zur Beförd. des Gartenbaues, 1834), and found a retrogression of temperature amounting to $2^{\circ} \cdot 2$ Fahrenheit, from the 11 th to the 13th of Maywhich, be it noticed, is about the time when the most rapid rise of temperature might be expected. Humboldt, in his Cosmos, speaking of this anomalous cold, says, ' it is much to be desired that this phenomenon, which some have felt inclined to attribute to the melting of ice in the north-east of Europe, should be also investi- 
gated in very remote spots, as in America or in the Southern Hemisphere,' which since his day has been accomplished, as we shall presently see.

We have to note also that the peculiarity, besides being observed in widely different places, has been observed at widely different times. Indeed, perhaps the most remarkable circumstance about these cold spells is that not only their occurrence, but the time of their occurrence, should have been noted by the unscientific, not usually ready to compare the weather and seasonal changes of one year (at least, in details) with those of another. Thus the three cold days of April, which before the change of style came early in the month (and were, in fact, for two or three centuries practically coincident with the first three days of the month), have been long known in Scotland and the north of England as the 'borrowing days'-that is, the days in reference to which there had been a borrowing, according to an old saying, embodied in the following doggrel lines:-

March borrows from April

Three days, and they are ill;

The first of them is wan and weet,

The second it is snaw and sleet,

The third of them is a peel-a-bane,

And freezes the wee bird's neb tae stane.'

I In the Glossary of Scotch Words and Phrases these lines arw given:-

Said March to April,

Gie me three hoggs upon yon hill,

And in the space of days three

I'll find a way to gar them dee. 
It is hardly necessary to remark that a phenomenon which thus attracts general attention, and is also capable of being verified by scientific observation, must be at once marked and very regular in its recurrence. Humboldt mentions that the cold days of May were recognised by the unlearned, and speaks of them as the three 'ill-named days' of May, corresponding with the days of St. Mamert (May 11), St. Pancras (May 12), and St. Servatius (May 13).

On the other hand, it must not be overlooked that these cold spells are not recognised every year. Take, for instance, the cold days in May, and consider the

The first a bitter blast did blaw, The second it was sleet and snaw ;

- The third it cam sae full a-freeze, The wee birds' neb they stack to the trees;

But when the days were past and gane

The three puir hoggs cam hirplin' hame.

But the following is probably a more perfect version of the doggerel poem:-

March said to Aperill,

I see three hogs upon a hill;

But lend your first three days to me, And I'll be bound to gar them dee. The first it shall be wind and weet, The next it shall be snaw and sleet; The third it shall be sic a freeze Shall gar the birds stick to the trees; But when the borrowed days were gane The three silly hogs came hirplin' hame.

Here the reference to the borrowing is clearer, for in the former version not days, but hogs, were borrowed. At the date to which the latter poem is usually referred, the three cold days of April were called April 1, 2, and 3, so that, being cold and bleak, they might well be regarded as borrowed by March. 
evidence in Gilbert White's weather records, in the Natural History of Selborne. In 1771 he tells us of spring weather and rain to the end of the first fortnight in May; in 1772, the first fortnight of May was dry, with cold, piercing winds; in 1773, throughout May and June, 'warm showers'; in 1774, no marked peculiarities; in 1775, cold weather throughout May; in 1776, cold weather throughout May; in 1778, a rainy May; warm Mays thence till 1782, when the first week of the month was dark and cold; in 1783 there was thick ice on May 5; in 1784, cold, dry weather during the first twelve days of May; in 1785, mild weather during the first twelve days of May; in 1786, mild weather during the first seventeen days; on May 1 and 2, 1786, 'thick ice'; in 1787, fine bright weather to the $22 \mathrm{nd}$; in 1788 , a warm, dry May; in 1789, a warm, wet May; and lastly, while May in 1790 and 1791 was warm throughout, May in 1792 was cold and bleak. It will be seen presently that these variations in the weather of May are as important a part of the evidence as the average depression of temperature on the 'cold days.

With reference to the cold days of February, the evidence is even more remarkable, as well for wideness of distribution in space, as for the length of time during which the phenomenon has been noticed. M. St. Claire Deville, searching meteorological records for evidence respecting the cold week in February (which he had found to be noted throughout Europe and in America), actually found that it had been noted in 
observations by the pupils of Galileo. These observations extend from 1655 to 1670 , and show that the minimum temperature was reached at that time on or about February 12. Mr. Russell, Government Astronomer at Sydney, has pointed out that the same peculiarity is observable in Australian registers.

It was natural that in searching for a cause of these remarkable anomalies of temperature, science should have been led to look outside the earth. Fortythree years have now passed since Adolph Erman threw out, in the Poggendorf Annalen, the idea that the sun's conjunction with the August meteors on February 7 and with the November meteors on May 12, might explain the cold spells which occur in February and May. He supposed that the ring of meteors through which the earth passes in August is smaller than the earth's orbit, so that, as the plane of the ring cuts the plane of the earth's orbit in a straight line, passing through the sun, and extending on one side to the earth's place on or about August 11, this line must extend on the opposite side to the place occupied by the earth on or about February 7, passing through the meteors before reaching the earth. The meteor ring, according to this view, would lie between the earth and the sun on or about February 7, and the earth being in their shadow would be to a certain extent chilled. So with the November meteors. The earth would pass into their shadow, according to this ingenious theory, on or about May 11 or 12 . Hence the ' cold days in May.' 
The theory is very ingenious, and has the advantage of being easily understood. It has therefore been quoted again and again, by persons unfamiliar with astronomy; it has done duty in newspaper science almost every year since it was first propounded. Even so late as 1879, M. de Fonvielle, editor of La Nature, presented it, not as a doubtful theory, but (after the manner which is characteristic, and I cannot but think a characteristic defect of French popular science teaching) as a known fact, which his readers were to accept because he said it-'Je vous le dis-Mor,' he seems to say; 'The chilliness is due to the fact that the earth passes behind a ring of asteroids, which absorbs a portion of the sun's warmth, due to us while he remains above the horizon. The temperature does not resume its ascensional movement until the annual rotation has carried our earth from the shadow of the multitude of small planets which is always projected on the same point of our orbit.'

Now, when Erman first advanced this theory, there seemed to be considerable evidence in its favour, and there were no known objections, at least of any great weight, against it. The case is very different now. Erman's theory, as he presented it, is absolutely untenable by anyone acquainted with what has been learned respecting the August and November meteor systems since 1866 .

The theory requires that the August and November meteor systems should pass between the earth's orbit and the sun, where they cross the plane of that orbit 
opposite the crossing places through which the earth herself passes on August 11 and on November 13. But we know now the precise form of each system, and we find that the other crossing place of the November system lies some nineteen times farther from the sun than the earth's orbit, while the August system also crosses the plane of the earth's orbit, much farther away than the earth travels from the sun. In other words, although rays from the sun towards the earth on or about February 7, and again on or about May 12, do undoubtedly fall upon the August and November meteor systems, they so fall not on their way to the earth, as Erman supposed, but long after they have passed the earth. As I wrote in Knowledge for May 26, 1882: "To charge, then, the August or the November systems with robbing our earth of a portion of its supplies of solar heat is to act like the wolf in the fable, who accused the lamb of troubling the stream, though the stream flowed from the wolf towards the lamb.' Our earth may intercept some of the supplies of solar light and heat passing out towards the November and August meteor systems, bat assuredly neither of these systems can cut off any of the supplies sent out to the earth.

It is noteworthy, indeed, and I think surprising, how little the topography, so to speak, of the August and November meteor system seems to be understood even by authors who, though writing popularly, are supposed yet to understand what they are writing about, and in fact to have no other object in writing 
but to explain to others what they thoroughly understand themselves. For instance, in Guillemin's $L_{e}$ Ciel, and in the English translation edited by Mr. Lockyer, there was given a stupendously impossible theory of the November and August meteor showers as produced by a single ring of meteors, of which a picture was presented, in which this single ring was shown cutting the earth's orbit in two places, though it was perfectly well known, long before the discoveries of 1866 and following years, that both meteor systems cut the earth's path at an angle. The idea of the August meteors crossing the plane of the earth's orbit at a sharp angle (as, indeed, at any angle) when the earth is in August, and then crossing the same plane (necessarily in a contrary direction) at the place occupied by the earth only three months later, is, of course, outrageously absurd. Yet here two professed teachers of the general public-rather condescending teachers too-not only describe this impossible movement, but even endeavour to picture it. If they had attempted to show it ' in elevation' as well as 'in plan,' they could not have failed, I should imagine, to recognise its absurdity; but without that, the absurdity should have been obvious.

To return to Erman's theory, it by no means follows from the disproof of the theory as advanced, that the anomalous falls of temperature in February, April, and May are not due to the interposition of flights of meteors:

Yet the objections even to the general theory are 


\section{LIGHT SCIENCE FOR LEISURE HOURS.}

weighty. If meteor streams lying between the earth and the sun diminished our supply of heat, we should expect that some among them would be visible upon the sun's face at those times, when duly magnified by powerful telescopes. A distant flight might, indeed, escape without any of its individual members being detected. But even then one would expect a measurable decrease of the sun's brightness, and hitherto nothing of this sort has been recorded by scientific observers.

But after all, as I pointed out in the paper above referred to (Knowledge, for May 26, 1882), the terrestrial test of Erman's theory is the best. If meteoric bodies come between the earth and sun at any time, in such numbers as to make us feel cold in their shadow, they must cool the whole earth, not England, or Europe, or the northern hemisphere. 'If, then,' I wrote at that time, 'in a careful comparison of the mean daily temperature at observatories all over the earth, it is found that the cold snaps of February, April, and May are everywhere to be recognised, then it must be at least admitted that the cause of the peculiarity is to be sought outside the earth herself.'

This, which seemed to me unlikely when I wrote those lines, has now happened. There seems no reason to doubt that the relatively cool weather of February in the southern hemisphere (I say relatively because, of course, February belongs to the warmest part of the southern year, corresponding to our August) is not coincident by a mere accident with the cold 
weather of our northern February. And the same with the cold days of April and May.

We seem obliged, then, to look for some cosmical cause of the anomalous falls in the earth's temperature; and as it is difficult, if not impossible, to conceive any cause which could directly affect the sun's temperature on special days of the terrestrial year, we seem naturally led to infer that somewhere between the earth and the sun there lies either constantly or usually something of the nature of a cloud which intercepts a portion of the sun's light and heat. If we consider for a moment what any special part of the earth's year means, we seem forced to this conclusion. The 9 th of February, for instance, is the time when, in her course around the sun, the earth arrives at a particular part of her path ; her coming there can in no way affect the sun's light and heat, which therefore cannot be diminished at that date (systematically) any more than at any other time. Yet the heat received by the earth when she gets there is less; hence it seems to follow inevitably that the earth there passes through a region where less heat (and therefore less light) is received because of some shadow-throwing matter.

Now, when Erman enunciated his theory of the interposition of meteoric streams, it was natural that such meteor systems as those which produce the August and November showers should be regarded as the shadow-throwing matter. Those were the only important meteor systems recognised, and there was no known reason for supposing that many such systems exist. 
But at present our ideas about the meteoric components of the solar system are very different. While we know certainly that neither the August nor the November systems can throw the earth into shadow in February and May, we know just as certainly that there are meteor systems-myriads, indeed, of meteor systems- which are much better fitted to cloud the solar rays than are those two.

In the first place, we know that the August and November systems are simply those two meteor streams, or incomplete rings of meteors-among several hundred such systems through which the earth passes-which chance to be so situated as to produce the most conspicuous and remarkable starshowers. There are doubtless many among the hundreds of others which are far more important numerically, and as regards the size of their individual components, than are these two. But others we merely skirt, or we have never fairly gauged, because the earth has not yet chanced to pass through their richer portions. These two the earth has passed through more centrally (though we do not even yet know that the earth has passed through the centre of either), and it has also happened that the earth has passed through those parts of these systems which are most richly strewn with meteors-the gemmed region of the meteor-ring-though, again, we do not yet know the true wealth of either system. To suppose that those of which we know only by passage through their outskirts are of inferior wealth, that the two we 
do know are necessarily the richest, is to suppose what, according to the laws of probability, is exceedingly unlikely.

But we also know that the earth can pass through, centrally or skirtingly, but a very minute proportion of the meteor systems which really exist in the solar domain. Picture the earth's path around the sun as what it really is, a mere thread-like ring of space around the sun, having a circular cross-section with a diameter of less than 8,000 miles (a mere nothing), while the diameter of the ring itself is no less than 185,000,000 miles, and we say how exceedingly minute is the space swept each year by the earth, in comparison with the sun's domain even to the earth's distance, and still less with the entire spherical region enclosed within the orbit of Neptune. If we lived on another planet-Venus, for example-we should become aware, there also, of multitudes of meteor systems, not one of which the earth passes through. Another set of entirely new meteor systems would come within our ken if we transferred our abode to Mercury or to Mars, or to any of the giant planets which travel outside the zone of asteroids. But even all the planets together do not actually traverse (in the way essential to meteoric encounter), do not, as it were, sweep through more than a very minute portion of the solar domain. Assuming within the orbit of the earth the same degree of meteoric wealth that the earth encounters in each of her annual circlings, we should have to believe in millions-nay, in millions of millions-of meteoric 
systems, passing through the region of space lying nearer to the sun than the sun's orbit.

But even this is far from being all. We have every reason to believe, in fact it may be said to have been to all intents and purposes demonstrated, that the wealth of meteoric distribution increases greatly within the earth's orbit, increases much more rapidly within the orbit of Venus, still more rapidly within the orbit of Mercury, and most rapidly of all in the sun's immediate neighbourhood. The proof of this has, of course, not been derived directly from meteoric observation, for we can become directly conscious of no meteor systems but those through which the earth actually passes, nor even of these save by those occasional transits through them during which a few thousands of individual meteors are, as it were, swept out of them by the onward rushing earth. Yet by reasoning of obvious force, the existence of multitudinous meteor systems, growing ever more numerous and ever richer, towards the sun's neighbourhood, has been clearly established. It has been found, first, that meteor systems follow in the course of comets; it has next been shown that when the earth passes through the track of a comet meteoric bodies in great numbers are encountered; it may be inferred then, first, that every meteor system follows either a comet now existing or one which, like Biela's, has been dissipated by processes whose nature is not as yet known; ${ }^{1}$ and,

' It so happens that one of the most remarkable meteoric showers ever seen-the only one which was ever predicted before it had been 
secondly, that every existing comet is followed by a train of meteors (which we may well believe to be in some degree proportional in wealth of distribution and, as respects the size of its components, to the comet with which it is associated). Now it is further found that the number of cometic paths increases rapidly as we approach the sun; and not only is the rate of increase rapid, but this rate is itself increasing as the sun is approached, so that whatever the wealth of cometic, and therefore of meteoric distribution, at the earth's distance from the sun, the wealth of such distribution close by the sun is enormously greater.

But we have other evidence on this point which, though perhaps by itself it might not be very weighty, amounts almost to demonstration when regarded as interpreting, and also as interpreted by, the evidence we have just been dealing with.

The solar corona might in former days have been regarded as in no way connected with the subject we are considering ; for in former days, despite the really recognised by annual star-showers-was due to meteoric bodies following in the track of a comet already dissipated, viz., Biela's (or Gambart's, as it is more properly called). This comet, which was divided into two in 1846, was looked for in vain in 1866, and when next due, in 1872; but because then it should bave passed the earth's track (which it chances nearly to cross) shortly before the earth arrived near the passing-place, some students of astronomy (I was one of them, and, I believe, the first) suggasted that, in all probability, when the earth arrived at that part of her orbit about Norember 27 , there would be a display of meteors, radiating (becanse following the comet) from a part of the star-sphere near the feet of Andromeda ; and such a display was seen (one of the richest star-showers of the century, numerically) on the night of November 27 , and with the predicted 'radiant point.' 
perfect evidence already existing to show that the corona belongs to the sun, many students of astronomy seemed to regard it as a part of scientific caution to close their eyes to the evidence, and regard as at least tenable the hypothesis that the corona may be a lunar or a terrestrial, or even a merely optical phenomenon. But within the last ten or twelve years the corona has been recognised by all for what (as I pointed out in 1869 ) it might have been known to be, centuries ago, a distinctly solar appendage. So understood, however, the corona's nature yet remained to be interpreted. To some it appeared as a sort of solar atmosphere, to others as a magnified aurora, while yet others regarded it as due to the constant emission of matter from the sun under the action of repulsive forces akin to those by which the tails of comets are supposed to be produced.

I pointed out long since, and with each year since the evidence for that view has become clearer and more decisive, that whatever other theory of the corona we may accept we cannot reject the belief that a part at least of the coronal light is due to meteoric matter travelling around the sun in streams and systems like those which produce the August and November star showers, but much more closely aggregated. When we take into account the much greater wealth of meteoric matter near the sun, that such matter is very much more brilliantly illuminated than meteoric matter at our earth's distance, while a portion of it is in all probability rendered self-luminous, if not actually 
vaporised under the sun's heat, we see that even if no such phenomenon as a total eclipse of the sun had ever been seen, one could predict that when the sun's light was intercepted by an extra-terrestrial body like the moon, a glory of light such as the solar corona would be seen around him. Those multitudinous meteoric streams in his neighbourhood, lit up by a splendour compared with which that of our sun at noon is almost as darkness, could not fail to be conspicuous around the globe of the sun, so soon as his own splendour was shielded from us by the interposed body of the moon. As I wrote, in my treatise on the sun in 1870 , before the true nature of the corona had been generally recognised, so I write now when none are in doubt as to the corona being a solar and not a lunar or terrestrial appendage - 'We have two distinct lines of argument: we are led by the consideration of the phenomena actually presented by the corona to the conclusion that multitudes of bodies too minute to be separately visible exist around the sun; while we have been led by the consideration of what we know respecting multitudes of minute bodies actually travelling around the sun, to the conclusion that a corona or aureole of light would be seen around him during total eclipse.' This being so, we cannot hesitate to accept, as at least partially explaining the phenomena of the solar corona, the theory that its lustre is in great part due to the streams and systems of meteoric bodies travelling around the sun, in his immediate neighbourhood.

But we have next to notice a peculiarity of the III. 
corona which can only be explained as due to meteoric streams, and which, considered in connection with what we have already learned, seems at once to point to a probable and, if demonstrated, a most interesting explanation of the anomalous temperature changes. I refer to the existence of certain rays or streamers, as they have been called (rather from their appearance than from anything which has been proved respecting their nature), extending from the eclipsed body of the sun as the brighter and farthest reaching portions of the solar corona.

During the total eclipse of July 1878 Professor Cleveland Abbe, observing the eclipse from a station on Pike's Peak (not at the summit, but high above the sea level), was able to trace a long, seemingly radial streamer to a distance of no less than six diameters of the sun, or about five million miles from the sun's disc. Four other rays were visible extending not quite so far, but the stoutest of them reach fully two million miles from the edge of the sun, assuming its length to lie at right angles to the line of sight; if, which is far more probable, its length was inclined to that line at an acute angle we must adopt a higher estimate.

Now these rays had well defined edges, and their brighter portions were not by any means radial extensions from the sun. Again, while two grew narrower with increased distance from the sun, the other two (which were apparently the prolongations of the former, on the opposite side of the sun) grew broader with increase of distance. It seems quite impossible to 
explain these streamers as formed of matter extending outwards from the sun as from a centre.

Mr. Cleveland Abbe, himself, came very quickly to the conclusion that these objects were meteor systems. 'Meteor streams!' he said, 'there is the key to the sqlution: not such meteors as some suppose to be falling into the sun daily, but the grand streams of meteors that cause the numerous shooting stars of August and November, and of the existence of which there is indubitable proof. These streams consist of fine particles or pieces, each a long way from its neighbour, but all rushing along in parallel orbits about the sun (that is, all belonging to any given system) like the falling drops of rain in a thunder-shower. Such a stream as the August meteor system, when far beyond the sun, but still lighted up by it, would reflect to us a faint uniform light precisely like that of these rays. If one end of the stream were further from us than the other, the effect of the perspective would be to produce a tapering or wedge-shaped appearance. In some other part of our orbit, or with the meteor stream in some other part of its orbit, the perspective might vanish and the two ends appear of the same width. In this way,' Mr. Abbe proceeds, 'we shall undoubtedly be able to explain the very numerous historical and memorable occasions on which flaming coronas, swords, comets, \&c., seen in the sky during a total eclipse, have been regarded by the superstitious as divine omens.'

Now here it must be noted that while all this may be true of some meteor systems, it is assuredly 


\section{LIGHT SCIENCE FOR LEISURE HOURS.}

not true either of the August meteor system or of the November one. Neither of these systems could be discernible at all during any total eclipse of the sun; for the simple reason that neither is rich enough in meteoric matter nor illuminated with sufficient brilliancy by the sun. We can be certain that such meteor systems could not be seen during a total eclipse, if we take into account a simple consideration which Mr. Abbe seems to have entirely overlooked. If a meteor system like the November system, which has its greater part outside the orbit of the earth, could be seen at all it would be seen during the darkness of midnight. Let me be understood. I do not mean that on every night, or even at any given season in each year, we might expect to see the November or August meteors. But this is certain, from the known position and movements of these systems, that on many occasions during the last century (to go no further back) they should have been seen far more conspicuously on the midnight sky-that is, on the side of the stellar sphere opposite to the sun-than they ever could be seen during total eclipse. In one case we see them when we are nearer to them by a whole diameter of the earth's orbit (or 185,000,000 miles) illuminated fully like planets in opposition, and on a dark background upon which stars down to the sixth or seventh magnitude are visible to the naked eye. In the other case, we look at them athwart the place of the sun, and on a sky which, though it seems dark by comparison with the brightness of mid-day, is yet very 
different indeed from the dark sky of midnight (as is shown by the circumstance that during the darkest total eclipse of the sun no third magnitude star has ever been seen, and only the brightest stars of the second magnitude). Moreover, during a total eclipse it is only on parts of the sky remote from the sun that stars are seen; close to the sun the brightness of the corona hides the stars from view. But it is precisely in this brighter portion of the sky during total eclipses that these streamers have been most favourably seen.

It is absolutely certain, in point of fact, that if these streamers are due to meteors, of which I have for my own part very little doubt, the systems to which the meteors belong are close to the sun where we see the streamers. The mere fact that the streamers are bright near the sun's place proves this. Imagine a meteor system at the earth's distance from the sun, seen athwart the sun's place during a total eclipse. Then it is true that from the parts of the system lying apparently nearest the sun, the greatest quantity of light would be reflected toward us, but the diminution in the quantity of light would be very slight, and would be more than compensated by the darkening of the sky with increasing distance from the central and brighter parts of the corona. Apart from this, the line of sight would be directed through a longer range of meteors, the greater the distance of any part observed from the sun's place. A streamer produced in this way would be more conspicuous the farther the part observed was from the sun. The reverse, however, is 
the case, and in such marked degree that no streamer can be traced at all to a distance of more than five or six diameters of the sun from him. In this we have proof positive, that there is a marked falling off in the illumination of the meteor stream on either side of the sun's place, wherefore (not as a doubtful inference, but with absolute certainty) the parts thus much more faintly illuminated are much farther from the sun.

No other argument should be necessary, but so slowly are considerations of this kind attended to, that it may be as well to strengthen the evidence which, rightly apprehended, is irresistible. Here, then, is another argument:- -

If meteor systems lying as far as the earth's orbit from the sun, or even no farther from him than the orbits of Venus or Mercury, could be seen during total eclipse, the chances would be greatly in favour of such a system being seen as a thwart streak not passing directly (in appearance) behind the sun's disc, but lying above or below or on either side of that disc. The meteor stream might be compared to the orbit of a planet, except that all the planets have orbits nearly in the same plane as the earth's, while the meteor systems cross the plane of the earth's orbit at all possible angles. Now even in the case of the planets Venus and Mercury, the orbits, though lying not far from the plane of the earth's path, lie always, except for a few days in December and June, in the case of Venus, and in November and May, in the case of Mercury, above or below the sun's disc as seen from 
the earth. So that nearly always if there were a meteor system travelling in the track of Venus or Mercury, we should see that system during eclipse (if at all) passing clear of the disc of the sun though near it. In the case of the meteor systems, we should see the earth stream most of the time (if we saw it at all) passing far away from the disc of the eclipsed sun. Take, for instance, the August and November systems. These, if visible at all, would only be seen as coronal streamers on or about February 7 and May.12, at other times they would be seen to pass far from the solar disc.

The fact, then, that during solar eclipses these meteor systems (if thus we are to explain the coronal streamers) seem nearly radial to the solar disc, is another proof, but the other was sufficient, that the part of the meteor stream producing the observed light, is in each case very close indeed to the sun.

Now it is clear that when we see a meteor stream as a radial, or rather diametral streak athwart the sun's place, the brightest part of the stream as so seen must lie really behind the sun. It looks like a double projection, or rather two projections, one on each side of the sun, but it is manifestly nothing of the sort, but a tract of illuminated matter extending across the space behind the sun.

In this respect I must correct the views expressed by Mr. Russell, of Sydney. He speaks of the meteor systems to which he (doubtless correctly) attributes the coronal streamers, as though they formed a solar appendage shaped as the corona seems to be shaped. 
'Astronomy has satisfactorily proved,' he says, 'that there are meteors enough in the solar system to produce the corona; and we all know that this coronal light is never concentric with the sun, and that it generally runs out in particular directions, and is never seen twice in the same form. Almost every drawing or photograph taken in eclipses proves that it is much brighter in some parts than in others, that is, that the matter which reflects the sunlight is not uniformly dense, and stops and reflects more of the sunlight proceeding in certain directions than in others; and any planet placed, so to speak, behind an expansion of the corona would suffer loss of light. If this coronal point (or projection) be, as they sometimes are, of enormous extent, there must be a corresponding loss of light and heat, and to such variations of sunlight the earth is beyond question subjected. There are hundreds of meteor systems cut by the earth's orbit which, as they all pass round the sun, must be concentrated (in his neighbourhood), and hence it is more than probable that when we look at an eclipse, there is just as great an extension of the corona towards the earth, where we cannot see it, as we see to right and left of our line of sight. Unfortunately, suitable eclipses come so seldom that the recurring forms of corona, which the fall of temperature in February, May, and at other dates seems to indicate, cannot be seen; but we know that the meteor streams have definite orbits about the sun; and therefore would place each year about the same quantity of meteoric 
matter between the sun and the earth at a particular date; and it must not be too readily assumed that the effect of it would be insignificant. Dull brown or black bodies, such as meteors, receive far more light than they reflect, and yet at solar eclipses, photographs of the light they do reflect can be taken in a very short time.' So that it is reasonable to admit at least the possibility that the intervention of meteors under ordinary circumstances may perceptibly affect the sun's heat and give rise to the sudden falls of temperature we have described.

Mr. Russell appears to suppose that in the corona there may be streamers projecting from the sun's surface directly towards the earth, and others on the side remote from us projecting directly from the earth. This, however, is not possible, so far as the meteoric corona is concerned. A dense meteor stream passing close by the sun's surface may, from particular points of view, appear to extend radially from the sun, but there is no position which the earth can possibly assume in which such a meteor stream would be directed radially from the sun towards us. The earth can be so situated that the rich part of a meteor stream may be pointed (so to speak) directly towards her; but whenever that is the case the meteor stream is removed to some distance from the line towards the sun. In point of fact, to suppose that a portion of a meteor stream can be radial from the sun in any direction whatever is equivalent to supposing that a part of an elliptical orbit around the sun can be 
in the direction towards or from the sun round which the body traversing that orbit is travelling, which is of course impossible.

I cannot, then, believe with Mr. Russell that the earth can ever come exactly opposite a streamer or projection of meteoric matter extending radially from the sun, simply because the position is demonstrably impossible. But this does not at all invalidate the meteoric explanation of the cold days in February, April, and May. It would doubtless be easier to believe in the effective shadows of meteors, if they could fall lengthwise along the space between the earth and the sun. But if two or three meteor streams should chance to lie between the earth and sun, not lengthwise but athwart, their effect in shading the earth might be quite sufficient to produce the fall of two or three degrees in temperature observed during the cold spells.

For it must be observed that the fall of temperature, if caused by meteoric interposition, would be due, not to the diminution of the sun's outpour of heat at any particular moment, but to the entire loss of heat during his passage past the interposed meteor stream, and that might last two or three days, affecting the entire earth, not only those places where day was in progress. The actual supply of heat at any moment. would of course only be diminished where the sun was shining; but even where the sun was not shining the effects of the diminution in the total terrestrial supply would presently be felt.

Let us make a rough calculation, however, to see 
whether the quantitative loss of heat-during, say, three days of the interposition of a meteor streamcould produce any measurable effect.

Suppose a meteor stream to be a million miles through, in the direction towards the sun (from the -earth), and to be so broad, supposed to be seen edgewise as to cover the whole breadth of the sun during three days of the earth's motion in her orbit. Then the region of meteor stream space actually intercepted between the earth and the sun, would obviously be a frustum of a cone, having the sun at its base, and the earth near its apex. But we need not trouble ourselves to consider this region as other than a cylinder, a million miles high. Supposing it to be near the sun (as we must consider any meteor system visible during a total eclipse to be), we may set the diameter of its base at about 800,000 miles; whence it follows that its volume would be about $500,000,000,000,000,000$ cubic miles. If we assume that there is but one meteor, one inch in diameter, in every 5 cubic miles, it would follow that at each instant during the three days' passage there are interposed between the earth and the sun (members of that meteor system) no less than $100,000,000,000,000,000$ meteors, each one inch in diameter. Being so near to the sun, these meteors would each hide a space equal to a circle-inch (that is, a circle an inch in diameter) of his surface from our earth's view. Now, in a circle mile there are nearly 1,000,000,000 circle-inches. Hence it follows that, apart from the very cases in which these widely 
scattered one-inch meteors would be in or very near the same line of view, they would hide from view a space equal to $100,000,000$ circle-miles, or a space equal to that which would be hidden by a planet 10,000 miles in diameter, close to the sun. This would suffice to cut off about a 7,000th part of his light and heat.

Now from the known wealth and extent of such systems as the August and November meteor streams (the latter of which follows in the train of a comet so small as to require a telescope to make it visible), and the enormous probability that others far richer exist in the space between the sun and the earth's orbit, we might safely infer that there are in the sun's neighbourhood many meteoric systems far richer than the one we have conceived above. Again, every system of meteors circling around the sun aggregates as its members approach the sun, and segregates as they recede from him, so that we may readily believe in a far greater wealth of meteoric distribution in a flight of meteors passing its perihelion, than the above suppositions involve. Yet, again, if we take into account the minute but more numerous components of a meteoric stream, we should have to admit a far more effective interruption of the sun's light than we have considered above. For instance, if we suppose that besides a meteor one-inch in diameter in the space of five cubic miles there were a million tiny bodies whose combined mass would only make up such another one-inch meteor, we should have to assign to these million tiny bodies, not the same light-obstructive 
effect as the one-inch meteor produced, though their mass is only equal to it, but an effect no less than onehundred times greater. ${ }^{1}$ So that by merely supposing two sphere inches of matter, one as a single globe, the other distributed in tiny grains each a hundredth of an inch in diameter, throughout five cubic miles of space, in a system having the extent we have considered above, we should have not a 7,000th part but more than a 70th of the sun's light and heat obstructed. This if continued for three days would correspond in quantity to the cutting off of the sun's entire supply of light and heat during more than one hour. The effects of such a sun-shadowing, even though distributed over three days, could not fail to be recognisable.

It should be added that the mere visibility of a meteor system during total solar eclipse implies that it must be many times more richly aggregated than any meteor system encumbered by the earth,- - shows in fact, that the meteor systems so seen must be at least as rich as we have supposed in the above reasoning.

Suppose, now, that two or three meteor systems chanced to be interposed in the way supposed above. Then the effects deduced would be doubled or tripled; and not merely a recognisable, but a marked loss of solar heat would ensue. If, for instance, it so chanced

1 Each would have a diameter of only one-hundredth of the diameter of the larger ; and, its diameter being one hundrod times as largo as theirs, it would hide a space on the sun's surface ten thousand times as great as the space hidden by each of the others; but as there are a million of them, they would collectively hide a space one hundred times as large. 


\section{LIGHT SCIENCE FOR LEISURE HOURS.}

that there were one meteor stream lying in or near the plane of the earth's orbit and close to the sun, while a little farther away from him there were another, crossing the plane of the earth's orbit at a considerable angle, - then, as the earth in her motion came behind the former, she would be several days in its shadow. Suppose now that in the course of this time she came behind the second or thwart system also. Then for a day or two, or perhaps three, she would be in the shadow of both these meteor systems. I am not by any means speaking wildly, but most moderately, in saying that small and widely scattered though the individual meteors might be, the effect of their interposition could hardly fail to be recognisable. Nay, oddly enough, the smaller the meteors were individually (with a distribution of so much meteoric matter per cubic mile) the greater would be the effect of their interposition.

But, it may be said, if there are meteoric streams such as these, capable of casting an effective shadow on the earth, these streams ought to be visible during total eclipse. Of course, individual meteors could not possibly be seen. A meteoric mass ten miles in diameter, close to the sun's surface, would be utterly invisible in the most powerful telescope ever yet made by man. What, then, would be the chance of seeing bodies whose diameters (even those of the largest of them) would be measurable by inches? But if a meteoric stream can cut off a measurable or recognisable quantity of solar heat, it must of necessity 
(however dark its substance may be) reflect a measurable or recognisable quantity of sunlight, when farourably placed for observation. Is there any evidence to show that this has happened in the case of the meteoric streams we have been considering above?

When should they be looked for? Manifestly, during eclipses occurring at the time when the earth is opposite, or nearly opposite, the part of her orbit on which such meteor streams cast their shadows. For instance, if the fall of temperature on or about February 7 is caused by the interposition of one or more meteor systems, then, during an eclipse occurring near August 11, say within two or three weeks on either side of that date, we should see meteoric streamers extending apparently diametrally from the sun (that is, forming opposite radiations really due to their lying behind the sun).

Now in the eclipse of July 28, 1851, Airy, the Astronomer Royal, noticed that the corona looked like a radiated cloud behind the moon. In the eclipse of July 29, 1878 (more favourably seen than any other has ever been), a bar of light was seen forming two radiations along the ecliptic, while athwart was another stream of light (both probably being meteoric streams). In the eclipse of August 7, 1869, four long radial streams were traced by General Myer to a distance of two or three diameters of the lunar disc. (He calls them straight, massive, silvery rays, seeming distinct and separate from each other, the whole spectacle showing as upon a background of diffused rose-coloured 
light). In the eclipse of August 18, 1868 , the shape of the corona resembled what was seen during the eclipse of July 29,1878 , nearly enough to correspond to the difference of time.

In fine, so far as the pictures and other evidence in my possession enable me to judge, ${ }^{1}$ all that is known about that part of the meteor stream region beyond the sun as seen during eclipses in July and August, and therefore lying between the earth and sun in January and February, corresponds precisely with what we should expect if the cold spell of February is due to the earth's passage through the shadows of meteor streams at that time. It so happens that we have no good pictures of total eclipses in October (corresponding to the April cold spell) or in November (corresponding to the cold days in May). I have very little doubt that when such eclipses come to be observed, similar appearances will present themselves.

It will form an interesting subject of study during future solar eclipses to determine how far the observed meteoric structure of the corona corresponds with the variations of the earth's mean temperature during the year-in other words, to determine the relative density of the meteor shadows in different months and in different days. One cannot but regret the more seriously, that owing to unwillingness to recognise the clearest mathematical evidence as to the real nature of the corona,

1 For illustrations of the corona, as seen on these occasions, the reader is referred to my treatise on the sun. This particular point is fully discussed, with abundant illustrations, in the pages of Knowlodge. 
the valuable opportunities for observation during the last quarter of a century were frittered away in absolutely unnecessary attempts to prove the corona to be what mathematical considerations had already shown that it is-a solar appendage.

Longman's Magazine for December 1882.

\section{CONDITION OF THE LARGER PLANETS.}

M. VOGEL's researches into the spectra of the planets were regarded by him as affording evidence unfavourable to the opinion that the planets Jupiter and Saturn are still so intensely hot as to shine in some degree with inherent light. Although it is not at all necessary for the general theory which I have adrocated respecting the condition of the larger planets that any portion of their lustre should be regarded as inherent, yet as Vogel's conclusion does bear to some degree on one of the arguments which have been urged in favour of this theory, the opportunity seems convenient for summing up these arguments and discussing briefly the considerations on which M. Vogel bases his objection.

I would remark at the outset that I do not by any means share the opinion of some who, in dealing with this question, and other questions of a like nature, have said that it matters very little what theory is adopted so that it is a convenient working hypothesis, a string, so to speak, on which to thread the observaIII. 
tions. It will be found that this method of viewing matters is never expressed except by persons who have fallen into the habit of accumulating observations without reasoning upon them-in fact, without utilising them. Observation is with them not a means but an end. It seems to me, or rather I may speak more confidently and say that the whole history of science proves, that the real value of observation and experiment lies not in themselves, but in what may be deduced from them. They are the raw material whence scientific knowledge is to be manufactured. It is not the object of a theory to afford a convenient means of classifying observations and also to suggest occasion for making them, but to educe their real significance; and the sole reasonable object of observations is to suggest the true theory and to afford the means of testing and rejecting false ones. To assert that it matters little what theory is suggested so long as it affords a convenient means of classifying observations, is as absurd in reality as it would be to assert that it matters very little in what manufacture raw materials of a particular kind are employed, so that the manufacture affords a ready means of sorting them away and making room for fresh stores of them. The object of manufacture is to make articles which shall have real value, and raw materials are only of use in so far as they can be employed in the manufacture of articles of such a nature. In like manner the object of theorising or reasoning is to discover actual truths, and observations are only useful in so far as they 
enable us to discover such truths. The mere observer who argues that observation and not reasoning is real science, may be compared to an organ-blower who should argue that his work, not that of the organist, constituted real music. The organist cannot play without wind, the manufacturer cannot get on without raw materials, and in like manner, Kepler would never have established his laws without the observations collected by Tycho Brahé, nor would Newton have discovered the law of gravity without the raw material collected by Flamstead; but as it is important in organ music that the wind be exhausted in melody, not in mere noise, and important in manufacture that the raw material be employed to make useful not useless articles, so it is and has been a matter of considerable importance whether observations have been idly worked up in false systems like those of Ptolemy or Descartes, or wisely used to ascertain the truth, as by Copernicus, Kepler, or Newton.

The theory which is now to be considered is this, that the planets Jupiter and Saturn are still in a state of intense heat, being at a much earlier stage of planetary development than our earth or those four companion orbs, Mercury, Venus, Mars, and the moon (in one sense more specially a companion than the others), which have been called the terrestrial planets.

At the outset it may be well to consider the evidence for the only other theory which has been advanced on the subject-the theory commonly accepted with apparently as little question as though it had 
been the result of long and profound investigation, had been tested in every possible way, had been weighed and not found wanting by all the ablest astronomers the world has known. This is the theory that Jupiter and Saturn are bodies in the same condition as our earth.

It is not easy to find any reasoning whatever bearing upon this theory. It would seem almost that so soon as Copernicus had shown that the planets do not travel round the earth as a centre, but the earth with the planets travel around the sun, the conclusion was at once adopted that the earth and the planets are of necessity bodies of the same nature ; and that, as no one was at the pains to question this doctrine, it became gradually regarded as one that had been established by demonstrative evidence. The few instances of anything like reasoning which $I$ have been able to find scattered here and there in books of astronomy amount to what follows:-First, because Jupiter and Saturn are planets, and the earth is a planet, therefore those planets are like the earth. (This argument is open to the objection that it begs the question, which is, Whether other planets resemble the earth.) Jupiter and Saturn are globes like the earth (also like the sun and moon). They rotate on their axes, and therefore if they are inhabited worlds like the earth, they have day and night, and in that respect are like the earth. They circle around the sun, and thus if they are worlds like the earth, they are like the earth in having a year; also in having seasons, since their axes are not perpendicular to the 
planes in which they travel. It would be absurd to suppose that globes so magnificent were made for no special purpose, but we can conceive no special purpose they can subserve except to be the abodes of life; therefore they are worlds like our earth (though the spn, constructed on a still more magnificent scale, is certainly not such a world, or the abode of life). Their moons are manifestly intended to make up to them for their remoteness from the sun (only, when we calculate how much light these moons reflect to their primaries we find that they supply but a small fraction of the amount we receive from our moon). The rings of Saturn were manifestly intended for the benefit of Saturn's inhabitants (though they only reflect light to the summer hemisphere of the planet, and besides turning their darkened side to the other hemisphere, cut off the whole of the sun's light for many months, in some cases for several of our years, in succession). The belts on Jupiter and Saturn may be likened again to our trade wind zones, to which, however, they bear not the remotest resemblance, whether we consider their condition at any given time, or the rapid changes they undergo from time to time. In fine, the arguments used by the few writers who have condescended to present even a show of reasoning in favour of the theory that Jupiter and Saturn resemble our earth in condition, amount practically to this-that, assuming all planets to be generally similar, Jupiter and Saturn are like our earth in general respects, in which case they also resemble her in several details. 
I do nat consider it necessary to discuss Whewell's theory that Jupiter and Saturn are intensely cold planets, because it is professedly based on the theory that they are formed of such terrestrial elements as would, if in the same condition as upon the earth, have the observed density of Jupiter and Saturn, and that these substances, being further removed from the sun, are correspondingly refrigerated. There is not a line of direct reasoning, either à priori or à posteriori, in Whewell's chapters on the larger planets-only reasoning which depends on the assumptions which had been made by those whom Whewell proposed to controvert. In fact, his theory may be regarded, and was probably regarded by himself, as merely a reductio ad absurdum of the unreasoning faith of those who had long held unchallenged the belief in the habitability of all the planets.

I proceed to indicate the leading arguments for the theory that Jupiter and Saturn are still intensely hot, noting first that I do not propose to discuss the details of the various arguments ${ }^{1}$ (which I have already done elsewhere), and secondly, that the arguments are not dependent one upon the other, but severally independent, so that if any seem weaker than the rest, the conclusion is not on that account invalidated, but the weight of evidence only pro tanto diminished. It is

I I may, perbaps, be permitted to remark here, that the details of many among the arguments here indicated will be found fully discussed in the nerr edition of my treatise 'Saturn and its System.' 
important to notice this, because many who, in examining a series of arguments, recognise, or suppose they recognise, some weakness in the evidence of one or other argument, are apt to infer that the conclusion is to the same degree invalidated as it would be if the arguments were dependent, and therefore each one essential to the establishment of the conclusion.

The first argument for the theory is that derived from the now accepted hypothesis of the growth or development of the solar system. It is rendered to all intents and purposes certain, as well from the evidence of the earth's crust as from that given by the movements of the sun, planets, asteroids, and satellites, that the solar system was developed from a former nebulous condition. The process of development may have been that conceived by Laplace in his nebular hypothesis, which may be described as the contraction theory, or that recently suggested by meteoric discoveries, which may be called the accretion theory, or, far more probably, the solar system was formed by combined processes of contraction and accretion. But in any case the planets as severally formed were intensely heated, partly vaporous, partly liquid bodies, the larger being the more heated. It is no longer supposed, as in Laplace's time was the case, that the outermost planets were fashioned first. They may have begun to be formed first-this, indeed, is altogether probable -but the vastness of their bulk suggests that they went on gathering in matter and contracting (forming in the process their systems of moons) long after such 
small planets as Mars or Mercury, though begun much earlier, had gathered in their entire substance. It seems indeed not at all improbable that neither Jupiter nor Saturn have quite passed through even the first stage of planetary development, the ring-system of Saturn being suggestive of matter as yet not completely worked-up, so to speak, in that planet's system. But whatever uncertainty rests on this question there is none as to the original intense heat of those larger planets. They must have been far hotter when first formed than was our earth at the corresponding stage of her development. Nor is it at all open to doubt that each stage of cooling would be much longer in the case of these planets than the corresponding stage of our earth's cooling.' Jupiter contains 340 times as much matter as the earth, so that if the two orbs were of the same density Jupiter would have a diameter seven times as great, and a surface about forty-nine times as great, as the earth's. He would radiate, therefore, if at the same temperature, forty-nine times

1 The argument here used was first advanced by Sir Isaac Newton. 'A globe of iron an inch in diameter,' he says, 'exposed red hot to the open air, will scarcely lose all its heat in an hour's time; but a greater globe would retain its heat longer in the proportion of its diameter, because the surface (in proportion to which it is cooled by the contact of the ambient air) is in that proportion less in respect of the quantity of the inclnded hot matter; and therefore a globe of red hot iron equal to our earth, that is, about $40,000,000$ feet in diameter, would scarcely cool in an equal number of days, or in about 50,000 years. But I suspect that the duration of heat may, on account of some latent causes, increase in a yet less proportion than that of the diameter; and I should bo glad that the true proportions were investigated by experiments.' Buffon (according to Bailly) made experiments of the kind, with results confirming Newton's opinion. 
as much heat; but he would have about 340 times as much heat to part with for each degree of cooling; hence his rate of cooling would be slower in the proportion of about 7 to 1. Jupiter appears actually to have a much greater volume than has been here supposed, his diameter exceeding that of the earth nearly eleven times and his surface exceeding hers about 115 times. This would still leave his rate of cooling slower in the proportion of about three to one. But inasmuch as it is certain that if formed of the same material, Jupiter, when at the same stage of cooling, would be much denser than the earth (because of his greater attractive energy), our assumption rather falls short of the truth than exceeds it. The argument next to be considered will sufficiently indicate this. To complete the present argument it is only necessary to add that the various stages of cooling through which our earth has already passed have certainly required hundreds of millions of years, wherefore the corresponding stages for Jupiter would require seven times as many hundreds, and the total period required by Jupiter to reach the earth's present condition of development would exceed the time during which our earth has endured, from her beginning until now, six times, even though Jupiter at his beginning were no hotter than the earth. As he was certainly much hotter, it may fairly be said that he would require thousands of millions of years to reach the stage which the earth has reached after hundreds of millions of years; and that, if the two planets were both fashioned at the same time, Jupiter must; still 
require thousands of millions of jears before he will have attained to that stage of planetary life through which our earth is now passing. Saturn would not be so far in the rear of our earth because his mass does not exceed hers so greatly. Still he contains nearly a hundred times as much matter, and must be regarded as in all probability, so far as this argument alone is concerned, hundreds of millions of years behind our earth in point of development.

The second argument is that derived from the small density of Jupiter and Saturn. Jupiter has a volume exceeding the earth's about 1,250 times, but a mass only exceeding hers 340 times. Saturn's volume exceeds the earth's 700 times, his mass only 99 times. Jupiter's mean density is therefore about one-fourth, Saturn's about one-seventh, of the earth's. Science no longer accepts the belief that either planet is formed in the main of different materials, spectroscopic analysis having demonstrated the existence of a general uniformity of structure throughout the solar system. Neither can science any longer admit the possibility that Jupiter and Saturn are hollow globes, experiment having proved that under the pressure exerted by the mass of either planet, a substance a hundred times stronger than the strongest steel would be perfectly plastic throughout the greater portion of either planet's interior, so that hollow spaces, if they could be formed for a moment, would fill up just as an open space formed for a moment by thrusting water on one side fills up as the water flows back to its normal position. 
We are forced then to believe that there is some cause at work to overcome the natural tendency of the planet's mass. Doubtless this cause is the same which operates to prevent the sun's mighty mass from concentrating, as it would, into an intensely dense globe, were its gravitating energies left unresistedviz., intense beat. The sun is, of course, very much hotter than Jupiter and Saturn; his heat, indeed, overcomes a very much greater contractive energy. But Jupiter and Saturn must be very much hotter than the earth.

The third argument is based on the telescopic evidence of the existence of a very deep cloud-laden atmosphere surrounding each of the planets Jupiter and Saturn.

It is first to be noticed, as respects this argument, that the general aspect of the belts of Jupiter (Saturn is too far off for similar appearances to be noted) indicates the presence of rounded masses of cloud floating in a deep atmosphere. These rounded masses can only be seen as such on the middle parts of the disc, but there their appearance shows unmistakably that they are really round - that is, not merely round in appearance, as a circle is round, but round as a globe is round. No one who has studied Jupiter with a powerful telescope can for a moment doubt that some at least among the cloud-masses which are seen in his dise are roughly globular in shape. It is sufficient if only one of these masses has really had such a shape, for though any number of flat objects may float 
in a sea which so far as they were concerned might be shallow, yet if it is known that a single object has floated in it which was not flat, but on the contrary had great length, and breadth, and thickness, we know that the sea must be a deep one. Some among the rounded clouds of Jupiter, which not only by their shape, but by their shading, indicate a globular figure, would, if actually globular, require an atmosphere five or six thousand miles deep at the very least. The atmosphere may not be so deep as that, or may be very much deeper. Certainly it would at once remove the difficulty last considered if we could suppose the cloud-bearing atmosphere of Jupiter to be thirteen or fourteen thousand miles in depth, for then the solid globe within would not differ very much in mean density from the globe of our earth. But supposing we assume, as the result of the actual telescopic aspect of the cloud-belts, the depth of the atmosphere to be but about 2,000 miles, which would be less than the apparently minute diameter of one of the satellites, we should even then find that under the tremendous pressure exerted by Jupiter's attraction the lower strata of such an atmosphere, if composed of any gases known to us, and at the temperature of our own air even in the torrid zones, would be simply compressed into the solid or liquid form. At least they could not continue to obey the laws which perfect gases obey under pressure. Assuming the pressure at the visible limit of the cloud envelope to be less than onethousandth part of the pressure of our air at the sea- 
level, then fifteen miles below that limit the pressure would be equal to that of air at our sea-level, fifteen miles lower one million times as great, and fifteen miles lower yet, or still only sixty miles below the visible limits of the cloud envelope of Jupiter, the pressure would be one thousand million times as great as at our sea-level. The density, if only the gases composing that atmosphere could remain as perfect gases, would be more than a million times greater than the density of the heaviest known elements. Of course there is no such pressure, no substance exists at that density, sixty miles below the visible limits of Jupiter's atmosphere, nor ten thousand miles lower yet., No gas could remain as such at ordinary temperatures beneath a pressure which would make it as dense even as water; and if strata could and did exist in Jupiter at the higher pressures and densities named, he would weigh many thousand times as much as he actually does. But we are again forced to the belief that, unless his atmosphere is made of substances altogether different from any with which we are acquainted, there must be some power at work to prevent the compression which would otherwise inevitably result from the tremendous attractive energy of Jupiter's mass. That power can be no other than the fierce heat with which his whole frame, his atmosphere (and all but the exterior strata outside the outermost cloud-layers) are instinct.

It appears to me that a fourth argument of very great force can be derived from the cloud-belts in the atmosphere of Jupiter and his brother giant, Saturn. 
The existence of well-defined belts is proof positive of the existence of different rates of rotational motion. For instance, we cannot explain our own trade-wind zones, without taking into account the different velocities due to rotation near the equator and in high latitudes-matter flowing towards the equator lags behind, matter flowing from it travels in advance, and in either case zones are formed. If a similar explanation could be given of the belts of Saturn and Jupiter doubtless they would be accounted for. But where are we to find the varieties of heat in various latitudes of either planet which could account for the multitudinous belts sometimes seen? or how, if the sun's slow action on these remote and large planets were in question, could we account for the rapid formation and dissipation of cloud-belts? The largeness of these planets is a point of importance to the argument, because the larger a planet the less, coteris paribus, is the variation of temperature for any given difference of latitnde measured as a distance in miles. If then we cannot look for the required differences of rotational velocity where we find them in our earth's case, it is clear we must turn to difference of rotational velocity on account of difference of distance from the axis, not at places in different latitudes, but in places at different levels. In other words, we must conceive that under the action of the planet's intense heat vaporous disturbances of the nature of uprush and downrush are continually taking place. Matter rushing upwards from low levels to high levels, where the rate of rota- 
tion is very much greater, lags behind, while matter rushing downwards is carried in advance, and thus cloud zones are formed.

A fifth argument is derived from certain considerations depending on the behaviour of sun-raised cloudmasses in our own air, both with regard to the progress of the day, and with regard to the progress of the year. We know that, speaking generally, the clouds change as the day progresses, and that this is specially the case in those regions of the earth where regular zones exist. The sun, in tropical regions, rises in a clear sky and quickly gathers clouds together; these remain till the afternoon, when they become dissipated (usually with violent disturbance, electrical and otherwise), and the sun sets in a clear sky. As seen from Venus or Mercury the cloud-belt would extend across the middle of the earth's disc, but would not reach to the edge, either on the west or sun-rising side, or on the east or sun-setting side. Nothing of the kind is observable in the cloud-belts of Jupiter. Not only do they extend right across (though becoming fainter near the edges because seen through deeper atmosphere), but cloudmasses have been known to remain, quite recognisable in contour, during many Jovian days, and even for forty or fifty of our own much longer days. So also with regard to the year. In Jupiter's case, indeed, the effect of annual changes in the arrangement of clouds would not be recognisable, simply because the planet's equator is nearly coincident with the plane of Jupiter's orbit. But in Saturn's case the inclination of the 
equator is considerable; so that, as seen from the sun, the equator passes far to the north and far to the south of the centre of the disc, during the summer of the northern and southern hemispheres, respectively: We should expect to find these changes accompanied by corresponding changes in the position of the central zone of clouds. Our terrestrial tropical cloud-zone, being sun-raised, follows the sun, passing north of the equator during our northern summer, until at midsummer it reaches the tropic of Cancer, and passing south of the equator during the southern summer, until at midsummer (December) it reaches the tropic of Capricorn. But instead of the mid-zone of Saturn behaving in this way, it remains always equatorial.

Another (the sixth) argument, and in my opinion an argument altogether irresistible, is derived from the changes which have taken place from time to time in the outline of the planets Jupiter and Saturn, unless observations made by most skilful astronomers, and with instruments of considerable power, are to be rejected as unworthy of trust. I refer in particular, first to the observations by Admiral Smyth, Sir R. Maclear, and Professor Peacock, of the reappearance of the second satellite of Jupiter a few minutes after it had apparently made its complete entry upon the planet's disc at the beginning of a transit; and, secondly, to the fact that Sir W. and Sir J. Herschel, Sir G. Airy, the Bonds and Coolidge in America, and several of the Greenwich observers, have recognised the occasional assumption by Saturn of what is commonly 
called his 'square shouldered' aspect. These observations are far too well-authenticated, and were made by observers far too skilful, to be open to doubt or cavil. They cannot possibly be explained except by assuming that the outlines of Jupiter and Saturn are variable to such an extent that the variations appreciably affect the figure of the planets. Such variations, involving differences of level two or three thousand miles, are utterly incredible, and in point of fact impossible, in the case of planets like our earth. The heat generated by such changes would of itself suffice to melt and in large degree to vaporise the crust for many thousands of square miles around the scene of upheaval or depression, so that we should thus have, but in another way, the heat which my theory indicates. On the other hand, such changes of outline in a planet whose apparent outline is not formed by its real surface, but by cloud layers thousands of miles above the real surface, are very easily explained. Nay, they are to be expected (though only as rare phenomena). We know that cloud-belts sometimes form, or are dissipated, rapidly on the face of the disc. Equally, therefore, they must sometimes form or become dissipated rapidly at parts of the planet so placed as to form the apparent outline. There would then be a rapid change of outline, such as must have occurred in the case of the apparent reappearance of Jupiter's second satellite. Slower changes in the cloud-belts would correspond to the changes of shape observed in Saturn's case, and would explain Schröter's observation that at times the outline 
of Jupiter has seemed to him irregular, as if the planet's surface were partially flattened. Other observations tending in the same direction, as peculiarities in the shape of the shadows of Jupiter's satellites on the planet, in the shape of Saturn's shadows on his rings, and so on, are of less weight perhaps than those already considered, but unless those who recorded them (including some of the most skilful observers known) were entirely deceived, such observations can only be fully explained by the great depth of the cloud-laden atmosphere which surrounds the giant planets.

Lastly, there is the argument derivable from the peculiar brightness of the planets Jupiter and Saturn. These planets might be so hot as to glow with an intense light and heat, yet no part of their light might be discernible, the deep cloud-layers simply cutting it off before it reached the outermost or visible cloud surface. Or this might bappen with all the rays except those which travelled the shortest way through the cloudlayers. In the former case we should perceive some of the inherent light of these planets, in the latter we should only perceive their inherent light in the central parts of the disc, which would therefore look brighter than the parts near the edge. This last is the phenomenon actually observed, but it does not of itself suffice to prove (though rendering it probable) that the light from the middle portion of the disc is in part inherent. Nevertheless the planet's surface might, as I have already said, be intensely hot, and yet no trace of the inherent light be perceptible by us. That, 
however, could only happen because of the existence of very deep cloud-layers entirely shrouding the glowing planet, and in this case, as the clouds would probably - like our own clouds-have a much higher reflective capacity than rock surfaces have, we should expect to find the planets Jupiter and Saturn shining much more brightly, though only by reflected light, than they would if their surface resembled that of our own earth, or Mars, or Jupiter. Now the following table from Zöllner's ' Grundzüge einer allgemeinen Photometrie des Himmels' gives very interesting evidence on this point:-

Snow just fallen reflects about . . 783 parts of 1000 of incident light;

White paper

Jupiter's surface

Saturn's surface

Uranus's surface

Neptune's surface

whereas 700

624

498

640

465

$\begin{array}{ll}\text {, } & \text { " } \\ \text {, } & , \\ , & , \\ \text {, }\end{array}$

White sandstone reflects only about 237 parts of 1000 of incident light ; Clay marl

Mars's surface

The Moon's surface

$\begin{array}{ll}" & 156 \\ " & 267 \\ \text { " } & 174\end{array}$

156

267

174

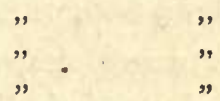

We may take Jupiter and Saturn together, and Mars and the moon; getting average reflective power of giant planets : that of small planets :: $561: 220$; or - the giant planets, if they owe their light entirely to reflection, have a reflective power more than $2 \frac{1}{2}$ times greater than that of the small bodies, Mars and the moon. As the sea regions of Mars are observably darker than his land regions, it is probable that our earth, if her light could be estimated in the same way 
(by an observer on Mercury or Venus) would be found to have a smaller average reflective power than Mars, her seas being so much larger.

We are forced by this argument to one of two conclusions-either Jupiter and Saturn shine in part by inherent light, or they are so thoroughly cloud-wrapped as to have a very high reflective power. Either conclusion would agree equally well with the theory I have adrocated, though, of course, the former would be much more effective, and would in fact be quite decisive in its favour.

For my own part, I think that the photometric evidence renders it very probable that a slight portion of the light of the planets Jupiter and Saturn is inherent; and I think the colour of the equatorial belt of Jupiter and its changes of colour correspond with this view. I should be disposed to assign, as the reflective power of Jupiter (his albedo, as Zöllner calls it), about 500, or more than twice the reflective power of white sandstone, and thus to attribute about onefifth of Jupiter's light to the planet's inherent lustre. (In Saturn's case Zöllner's observations are much less satisfactory-his measures indeed of the planet's total light were probably even more satisfactory than in Jupiter's case, but it is exceedingly difficult to take properly into account the effect of the ring-system, which, though very much foreshortened when Zöllner made his observations, must nevertheless have appreciably affected his results.) All the known facts accord well with this view. 
Certainly the spectroscopic evidence obtained by Vogel, or rather the general spectroscopic evidence (for his results are not new) is not opposed, as he seems to imagine, to the theory that the actual surface of Jupiter is intensely hot. His argument is that, because dark lines are seen in the spectrum of Jupiter, which are known to belong to the absorption spectrum of aqueous vapour, the planet's surface cannot be intensely hot. But Jupiter's absorption spectrum belongs to layers of his atmosphere lying far above his surface. We can no more infer-nay, we can far less infer-the actual temperature of Jupiter's surface from the temperature of the layers which produce his absorption spectrum, than a being who approached our earth from without observing the low temperature of the air ten or twelve miles above the sea-level could infer thence the temperature of the earth's surface. There may be, in my opinion there almost certainly are, layers of cloud several thousand miles deep between the surface we see and the real surface of the planet. I do not suppose that the inherent light referred to above as probably received from Jupiter, is light coming directly from his glowing surface, but the glow of cloud masses high above his surface, and illuminated by itperhaps even the glow of cloud-layers lit up by lower cloud-layers which themselves even may not receive the direct light emitted by his real surface.

To sum up, it appears to me, that a theory to which we are led by many effective and some apparently irresistible arguments, and against which no 
known facts appear to afford any argument of force, should replace the ordinary theory, originated in a haphazard way, and in whose favour no single argument of weight has ever been adduced. Since it appears(1) that if the accepted theory of the development of our system is true, the large planets must of necessity be far younger, that is hotter, than our earth and other small planets; (2), that if made of similar materials, those planets must of necessity be far denser than they actually are, unless they are very much hotter than the earth ; $(3)$, that the atmospheres (judging of their depth from the planets' appearance) would be compressed into solid and very dense matter under the planets' attraction unless exceedingly hot throughout their lower layers ; (4), that the belts and their changes imply the uprush and downrush of heated masses of vapour through enormous depths of atmosphere; (5), that the cloud-belts neither change with the progress of the day nor of the year in the large planets, but in a manner in no way referrible to the sun, and are therefore presumably raised by the intense heat of the planet's own substance; (6), that so remarkable are the changes taking place in the atmospheres of Jupiter and Saturn, as appreciably (even at our enormous distance) to affect the figure of those planets; and ( 7 ), that the planets shine with more than $2 \frac{1}{2}$ times the brightness they would have if their visible surface were formed of even so lustrous a substance as white sandstone-I think the conclusion is to all intents and purposes demonstrated that the planets Jupiter and 
Saturn really are in a state of intense heat. If they ever are to be the abode of life, they will probably not be ready to subserve that purpose for hundreds of millions of years.

Popular Science Revicw.

A GREAT SOLAR OUTBURST.

ON Sept. 7, 1869, at noon, Professor Young had been examining with the telespectroscope an enormous hydrogen cloud on the eastern limb of the sun. 'It had remained,' he says, 'with very little change since the preceding noon, a long, low, quiet-looking cloud, not very. dense or brilliant, nor in any way remarkable except for its size. It was made up mostly of filaments nearly horizontal, and floated above the chromatosphere with its lower surface at a height of some 15,000 miles, but was connected with it, as is usually the case, by three or four vertical columns brighter and more active than the rest. In length it measured $3^{\prime} 45^{\prime \prime}$, and in elevation about $2^{\prime \prime}$ to its upper surface' (that is, it was 100,000 miles long by 54,000 miles high). At halfpast twelve Professor Young was called away for a few minutes. At that time, 'there was no indication of what was about to happen, except that one of the connecting stems at the southern extremity of the cloud had grown considerably brighter, and was curiously bent to one side; and near the base of another at the 
northern end a little brilliant lump had developed itself, shaped much like a summer thunderhead.'

On returning, though less than half an hour had passed, Professor Young found to his great surprise that 'in the mean time the whole thing had literally been blown to shreds by some inconceivable uprush from beneath.' 'In place of the quiet cloud I had left,' he says, 'the air, if I may use the expression, was

FIง. 1.

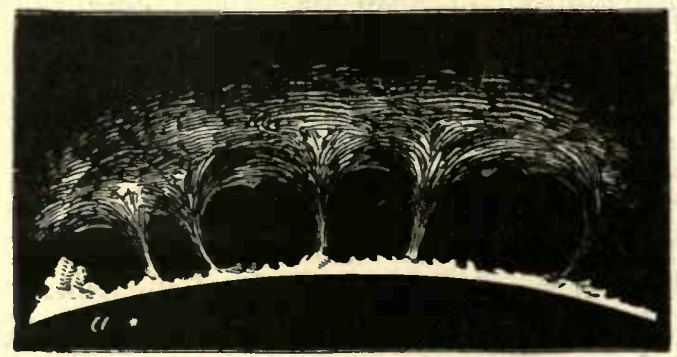

filled with flying débris-a mass of detached vertical fusiform fragments, each from $10^{\prime \prime}$ to $30^{\prime \prime}$ long by $2^{\prime \prime}$ or $3^{\prime \prime}$ wide, brighter and closer together where the pillars had formerly stood, and rapidly ascending. When I first looked some of them had already reached a height of nearly $4^{\prime}(100,000$ miles); and while I watched them they rose, with a motion almost perceptible to the eye, until in ten minutes $(1 \mathrm{~h} .5 \mathrm{~m}$. P.M.) the uppermost were more than 200,000 miles above the solar surface. This was ascertained by careful measurements; the mean of three closely ac- 
cordant determinations gave $7^{\prime} 49^{\prime \prime}$ as the extreme altitude attained; and I am particular in the statement because, so far as I know, chromatospheric matter (red hydrogen ${ }^{1}$ in this case) has never before been observed at an altitude exceeding $5^{\prime}$. The velocity of ascent ton.

Fig. 2.

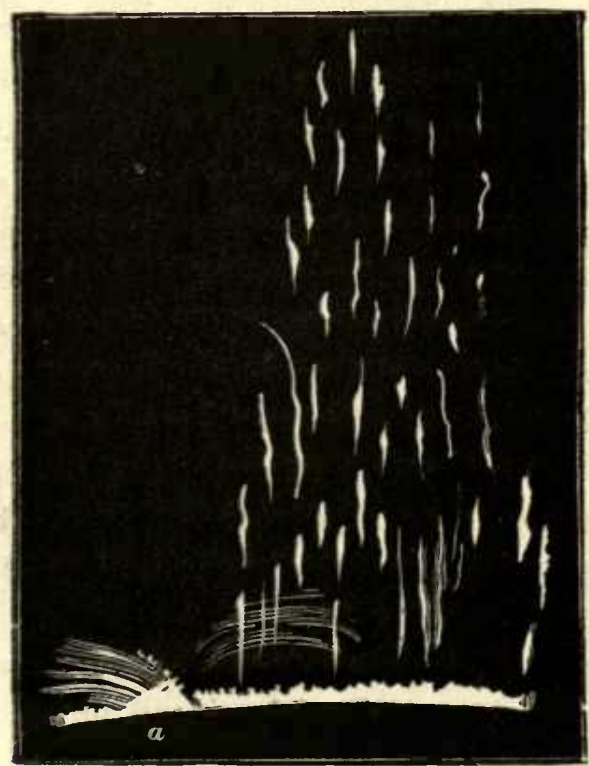

also, 167 miles per second, is considerably greater than anything hitherto recorded. A general idea of the

- Prof. Young prolably means that he was observing the red image of the cloud and uprushing matter-i.e. the image formed by rays eorresponding to the C-line of lyydrogen. Father Seceli mentions that he finds the indigo image (i.e. the image formed by rays eorresponding to the G-line of hydrogen) the most perfect and the fullest in detrils. 
appearance presented when the filaments attained their greatest elevation may be obtained from fig. 2 .

'As the filaments rose they gradually faded away like a dissolving cloud, and at 1 h. $15 \mathrm{~m}$. P.M. only a few filmy wisps, with some brighter streamers low down near the chromatosphere, remained to mark the place. But in the meanwhile the little "thunderhead" before alluded to had grown and developed wonderfully, into a mass of rolling and ever-changing flame, to speak according to appearances. First it was crowded down, as it were, along the solar surface; later it rose almost pyramidally 50,000 miles in height; then its summit was drawn out into long filaments and threads, which were most curiously rolled backwards and downwards like the volutes of an Ionic capital; and finally it faded away, and by 2 h. $30 \mathrm{~m}$. had vanished like the other. Figs. 3 and 4 show it in its full development; the former having been sketched at $1 \mathrm{~h} .40 \mathrm{~m}$., and the latter at $1 \mathrm{~h} .55 \mathrm{~m}$. '' 'The whole phenomenon,' he adds, 'suggested most forcibly the idea of an explosion under the great prominence, acting mainly upwards, but also in all directions outwards, and then after an interval followed by a corresponding inrush; and it seems far from impossible (the italics are mine) that the mysterious coronal streamers, if they turn out to be truly solar, as now seems likely, may find their origin and explanation in such events.'

'Professor Young mentions that his 'sketches' do not pretend to accuracy of detail, except the fourth, the three rolls in which are nearly exact. 
Now, it is to be noticed in the first place, that although the explosion thus described is the only one of the kind that astronomers have yet witnessed, we

Fig. 3.

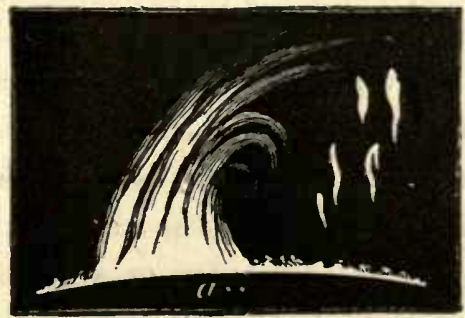

cannot safely infer that it was an exceptional solar disturbance. It is to be remembered that the sun is not always under spectroscopic surveillance, even in suitable observing weather, at American and European

FIG. 4.

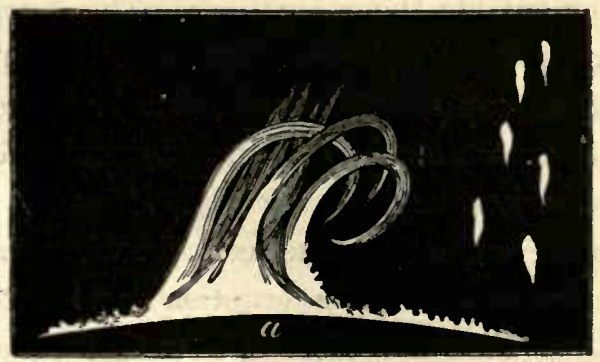

stations. Professor Young in America, and in Europe Lockyer, Janssen, Secchi, Respighi, and Zöllner, with the few others who take a more or less systematic part 
in the work, are unable to devote the whole of the day -or probably even a large portion of the day-to observation of the sun. But apart from this we must take into account the occurrence of unfavourable observing weather, and Lockyer speaks of days seemingly fine, when certain indications in the appearance of the prominence-lines assure him that observation is useless. Doubtless the experience of other observers resembles his in this respect. But this is not all. During a great part of the 24 hours the sun is not above the horizon at any of the European or American observing stations. And then, lastly, even when he is above the horizon, solar outbursts of enormous importance might take place without any possibility that terrestrial observers could become cognisant of the fact; simply because any outbursts in the central parts of the face turned towards the earth and of the half turned directly away from the earth, could not produce prominencephenomena outside the solar limb. The spectroscope gives us an account indeed of disturbances taking place on the sun's face; but the account can be by no means so easily interpreted as in the case of prominences seen in the ordinary manner.

When we combine these considerations with the circumstance that a solar eruption lasts but a few minutes, and that the observer is unable to examine more than one portion of the sun's limb at a time, so that many important eruptions might occur even while he was engaged in the most attentive observation, we see that outbursts like the one witnessed by Professor 
Young may occur very frequently and yet be very seldom seen. Again, the jet prominences seen by Respighi, Secchi, Zöllner, and others, though not. appearing to extend to the height reached by the hydrogen wisps watched by Young, may (many of them), have reached to an even greater height, being reduced by simple foreshortening; and as these are phenomena. frequently observed, we may not unsafely infer that. eruptions really as important as the one witnessed by Professor Young are by no means uncommon.

But let us consider what the facts observed by Professor Young really imply. This is precisely one of those cases where an observation requires to be carefully discussed in order that its full value may be educed.

Now the main point of the observation is this - that glowing hydrogen was observed to travel from a height of less than 100,000 miles to a height of more than 200,000 miles in ten minutes. To be safe, let us take the limiting heights at 100,000 miles and 200,000 miles; and let us assume that there was no foreshortening. These assumptions both tend, of course, to reduce our estimate of the velocity with which matter was ejected from the sun.

Now we need not trouble ourselves by inquiring whether the hydrogen wisps which moved upwards before Professor Young's eyes were themselves ejected, or whether their motion might not have been due to the ejection of other matter impinging upon these wisps and forcing them upwards. Some matter must 
have travelled at the observed rate - or (if the hydrogen was not itself ejected, then) at a greater rate.

The question which we have to deal with is therefore this-What must be the velocity of ejection in order that matter may pass between the observed heights in the observed time?

But it may seem that the problem might be simplified by inquiring what must be the velocity of ejection in order that a height of 200,000 miles should be reached. This, however, introduces the question whether that was really the limit of the hydrogen's upward motion. The wisps seemed to dissolve away at that elevation; but we cannot assume quite safely that the hydrogen there ceased to move upwards. On the contrary, it seems more likely that it neither diffused itself (so as to become invisible), nor ceased to ascend, at that level; but simply became invisible through loss of temperature, and therefore of brilliancy. It will be better, therefore, to take simply the flight between the observed.levels; for then we shall be attending solely to observed facts. We may, however, inquire as a preliminary process what would be the velocity of ejection necessary to carry a projectile (moving as if in vacuo) from the sun's surface to a height of 200,000 miles.

We find thus that a projectile must have an initial velocity of about 213 miles per second to reach the height certainly attained by the hydrogen wisps watched by Professor Young. ${ }^{1}$

1 The calculation is not difficult. The formula for our purpose may be thus expressed. Let $\mathrm{R}$ be the sun's radius or 425,000 miles; $H$ the 
Now the time in which a projectile with this initial velocity would traverse the upper half of its path is not so readily determined-in fact, the formula is not altogether suited to these pages. ${ }^{1}$ I must, therefore,

extreme height reached by a projectile from the sun; $V$ the relocity of projection. Then, a mile being the unit of length, and a second the unit of time-

$$
\mathrm{V}=379 \sqrt{\mathrm{H}}
$$

( 379 miles per second is the reloeity which would be required to carry a projectile away from the sun altogether); and we hare only to put for $\mathrm{H}, 200,000$ (miles), and for $\mathrm{R}, 425,000$, to deduce the required relocity.

${ }^{1}$ Following, howerer, the plan adopted in my treatise on The Sun, I gire the formula for all such cases in a note, so that those readers whose tastes are mathematical may nuake the calculation for themselves, if they wish to. It runs thus :-

$R$ being the sun's radius, $D$ the extreme distance of a projectile from the sun's centre, $\mathrm{X}$ its distance at time $t$ after starting from rest at distance $\mathrm{D}$ (from centre, be it remembered), then-

$$
\sqrt{\frac{379 \mathrm{R}}{\mathrm{I}}} \cdot t=\sqrt{\mathrm{I}) x-x^{2}}+\frac{\mathrm{D}}{2}\left(\pi-\text { rers. }^{-1} \frac{2 x}{\mathrm{I}}\right) .
$$

In the course of my examination of Professor Young s obserration, finding the application of this formula rather wearisome (especially as the formula had to be applied tentatively in dealing with the main problem, for it tclls us nothing as to the extreme height, when this is to be determined from the observed time between certain lerels), I was led to consider whether a geometrical construction might not be found which would at least afford a test of the calcnlative results. (For this, be it noticed, is the great ralue of geometrical constructions; they prevent any scrious errors of calculation, by affurding a tolerably close approximation to the truth; and in calculation-crede experto-great errors are most to be feared.)

I presently lighted on the following construction, which may be applied with singular ease, rapidity, and accuracy to all problems such as the one we are upon. Let $\mathrm{K} Q \mathrm{E} \mathrm{C}$ be a carefully constructed half cycloid, $\mathrm{K}$ being a cusp, $\mathbf{E}$ the vertex, and $\mathrm{E} C$ the axis. (The same cycloid is to be used for all problems, the remaining corstructions being peneillel.) Divide $\mathrm{CE}$ in $\mathrm{A}$ so that $\mathrm{CA}$ represents the sun's radius, 
ask those readers who do not care to make the calculations for themselves, to accept on trust my statement that $25 \mathrm{~m}$. $56 \mathrm{~s}$. would be the time required for the upper half of our projectile's course.

It is already obvious, therefore, that the matter watched by Professor Young did not behave like a projectile in vacuo, having 200,000 miles as the limits of its upward course. It traversed a space in 10 minutes which such a projectile would only traverse in about 26 minutes.

Now two explanations are available. We may suppose that the real limit of the upward fight of the hydrogen was greater than 200,000 miles, and that, therefore, the 100,000 miles next below that level were traversed with a greater velocity than would correspond to the case we have just been considering; or we may suppose that the matter was in reality projected with a much greater velocity than 200 miles per second, and was brought to rest at a height of 200,000 miles by the retarding action of the solar atmosphere cooperating with solar gravity. And of course we may conceive that these two explanations coexist, and that the two causes considered operate with any degree of proportional activity, between the relations which would

$\Lambda \mathrm{F}$ the fight of a projectile. About centre $\mathrm{C}$ draw half circle A D L, cutting half circle on $\mathrm{EC}$ as diameter in $\mathrm{D}$. Draw $\mathrm{D} \mathrm{M}$ square to $\mathrm{A} C$, and let $\mathrm{Mr} m \mathrm{~L}$, a half circle on $\mathrm{ML}$. cut $\mathrm{KC}$ in $m$. Then the time of descent from $\mathrm{E}$ to any point $\mathrm{P}$ in $\mathrm{E}, \mathrm{A}$ is represented by the ordinate $\mathbf{P Q}$ (parallel to $\mathrm{KC}$ ), where $m \mathrm{C}$ represents $18 \mathrm{~min} .40$ sec., which is the time in whieh $\mathrm{CA}$ would be traversed, with a relocity of 379 miles per second. 
make one or other the sole cause of the observed excess of velocity.

Now, to determine the actual height which must be reached by a projectile from the sun (in vacuo) so that it may pass from a height of 100,000 to a height of 200,000 miles in ten minutes, I have gone through $a^{t}$ series of calculations which need not be discussed here, leading to the result (which may be accepted as trustworthy) that 350,000 miles is the required height, and therefore 255 miles per second the requisite initial velocity. In this case the hydrogen wisps watched by Professor Young were in reality travelling at a rate of about 150 miles per second when they reached the highest visible part of their course and vanished from view as if by a process of dissolution.

On the other hand, it is not possible to determine the nature of the motion of hydrogen wisps, retarded by the resistance of the solar atmosphere, so as to travel. from a height of 100,000 miles to an extreme height of 200,000 miles in ten minutes. We are very far from knowing how to deal satisfactorily with the motion of a solid projectile through our own atmosphere, which may be regarded as appreciably uniform during the projectile's flight, the action of terrestrial gravity being also appreciably uniform. But in the case of the sula. atmosphere between the observed levels we have a problem infinitely more difficult, because the atmospheric pressure must be greatly less at a height of 200,000 miles than at a height of 100,000 miles, the solar gravity at these heights being also very different. 
Nor do we know what the atmospheric pressure is at either level. It would be mere waste of time to discuss a problem all the conditions of which are so vague.

But it will be worth while to consider the general relations which are involved.

In the first place, we may leave out of consideration the motion of the hydrogen before it reached the level of 100,000 miles. The retardation we have to inquire into is something taking place within the observed range of the projectile's motion, and we may consider the moving hydrogen precisely as though its motion had been due to some projectile force operating upon it when already at a height of 100,000 miles. Now we have seen that in order to traverse the next 100,000 miles above that level in ten minutes, it would require an initial rate of motion (at that level) sufficient to carry it to a distance of 350,000 miles from the sun's surface if unretarded. But as the matter (on the hypothesis we are considering) did not reach this distance (250,000 miles from its starting place), but, on the contrary, only traversed a distance of 100,000 miles before being reduced to rest, it is obvious that its initial velocity (at level 100,000 miles) must have been greatly in excess of the velocity which, at that level, would correspond to an upward range of 350,000 miles in all. In other words, the hydrogen, when at a height of 100,000 miles, was travelling much faster than a projectile would cross that level if projected in vacuo at a rate of 255 miles per second. So that 
leaving out of consideration all the retardation experienced by the hydrogen before it reached the level 100,000 miles, its motion at that level corresponded to an initial velocity much exceeding 255 miles per second. But, if the retardation was so considerable between the levels 100,000 miles and 200,000 miles, as to reduce the hydrogen to rest at the last-named level, whereas in vacuo it would have reached a level much exceeding 350,000 miles, how much more effective must the retardation have been in the first 100,000 miles of the hydrogen's upward course? It is difficult to express how much greater must be the average density of the solar atmosphere between the photosphere and a height of 100,000 miles, than between the height 100,000 miles and 200,000 miles; but the disproportion must be enormous. Apart from this, the retardation being always proportioned to the velocity (though the law of this proportion is not known), would have been much more effective in the lower part of the hydrogen's course, on this account alone. We have, then, this important conclusion (on the hypothesis we are dealing with), that after traversing a range of 100,000 miles from the sun's surface under the action of a retardation enormously exceeding that operating on the hydrogen in the observed part of its flight, the uprushing hydrogen still retained a velocity far exceeding that due to a velocity of 255 miles per second at the sun's surface in the case of a projectile in vacuo.

But we have now to consider towards which 
hypothesis we should lean, or rather which cause we should consider as chiefly operative.

In the first place, it is obvious that we cannot dismiss the hypothesis of retardation entirely, for glowing hydrogen travelling through an atmosphere even of extreme tenuity at an average rate of 167 miles per second must needs be enormously retarded. But I think that, apart from this, we cannot for a moment accept the belief that the hydrogen wisps which Professor Young watched as they slowly vanished at a height of 200,000 miles were then travelling upwards at the rate of about 150 miles per second. So acute an observer could not but have recognised the fact that the hydrogen was still in rapid upward motion at that time. We are compelled then, as I judge, to regard retardation as operative to at least some considerable degree in that upper half of the hydrogen's course.

This being so, I do not know that a single word of what I have said on the hypothesis of retardation being solely operative need be altered. The italicised words at the close of the remarks made on that view must still be used in stating the conclusion to which careful reasoning would lead us.

And here I approach the point to which these remarks have been tending. If we regard the hydrogen erupted or in motion in these jet prominences as not less dense than other matter partaking in the motion of primary ejection, the above conclusion, interesting as it is in itself, yet has no bearing on the subject of 
the corona. The erupted hydrogen reached a certain enormous altitude, and there (so far as the extrusion of matter from the sun was concerned) the work of the solar eruption came to an end. But the spectrum of the jet prominences indicates the presence of several other elements - amongst others, several metallic elements in the state of vapour. Now, it is highly probable that at a very early stage of the upward motion a large proportion of the metallic vapour would condense into the liquid form; and if so, such liquid metallic matter would thenceforward meet with far less resistance, and so would travel to a far greater distance than the hydrogen. But without insisting on this point, we may yet feel assured that under similar conditions of temperature and pressure the vapours of the metallic elements far exceed hydrogen in density. Thus they would from the very beginning of their upward course be exposed to a much less effective retarding influence. They would, therefore, retain a much greater proportion of the velocity primarily imparted to the whole body of erupted matter; nor is it by any means an unreasonable or unlikely supposition that at a height of 100,000 miles some of these constituents of the erupted matter would be travelling twice as rapidly upwards as the hydrogen watched by Professor Young. So far, indeed, is this view from being unlikely that it is difficult to entertain any other opinion. Yet, on this view, the matter referred to would be travelling at a rate greatly exceeding 400 miles per second; and a much smaller 
velocity would suffice to carry it away for ever from the sun's controlling influence. Much more, therefore, would the outrush of such matter suffice to explain the extension of the coronal streamers.

It would require only very moderate assumptions respecting the retarding influence of the solar atmosphere, to prove that the least of the jet prominences must have required a velocity of ejection competent to carry the vapours of metals as far as the outermost observed limits of the radiated corona.

\section{COMETS.}

OF all the objects with which astronomers have to deal, comets are the most mysterious. Their eccentric paths, their marvellous dimensions, the strange changes to which they are subject, have long been among the most striking of the wonders of astronomy. There is something specially awe-inspiring, too, in the thought of the gloomy domains of space through which the comet that visits our system for a brief time has for countless ages been travelling. Ordinary modes of measuring space and time fail us, indeed, in speaking of these wonders, or at least convey no real meaning to the mind. If the comet, for instance, which was in 1874 a conspicuous object in our northern skies be of 
this order-if, as our comet-tracker Hind suspected, its path in our neighbourhood is parabolic, so that either it has an enormously long period of revolution, or came to us across the interstellar spaces themselves-how useless is it to set down the array of numbers representing the extension of its path, or the years during which the comet has been voyaging through desert space! The comets indeed which come from the star-depths-and observation renders it all but certain that some have done so-cannot in any case have pursued a voyage less than twenty billions of miles in length, and cannot have been less than eight million years upon the road. That, too, was but their latest journey. From the last sun they visited to our own sun, such was their voyage; but who shall say how many such voyages they had pursued, or how many they will complete after leaving our sun's neighbourhood, before the time comes when some chance brings them near enough to a disturbing planet to cause their path to become a closed one? And even those comets which are now known to follow a closed path, returning again and again to the neighbourhood of the sun, need only be studied thoughtfully to present similarly startling conceptions. No matter what theory of their origin we adopt, we are brought face to face with the thought of time-intervals so enormous that practically they must be viewed as infinite. If we take the assumption that a comet of this order, which had been travelling on a path of parabolic or hyperbolic nature towards our sun, had been 
captured by the disturbing attraction of a planet, and compelled thenceforth to circuit on an oval path of greater or less extent, yet according to all laws of probability, how many times must it have flitted from star to star before it was thus captured! For the chances are millions to one against so near an approach to a planet as would ensure capture. But if, appalled by the enormous time-intervals thus revealed to us, we turn from that assumption, and find within the solar system itself the origin of the periodic comets, how strange are the theories to which we are led! Those comets which come very near to the sun may have had a solar origin; and those which approach very near the path of one of the giant planets may have been propelled from out of such a planet when in its sun-like youth. Even then, however, other comets remain which are not thus to be accounted for, unless we regard them as derived from planets outside Neptune, hitherto undetected, and perhaps detectable in no other way. And when we have taken such theories of cometary origin, not, indeed, for acceptance, but to be weighed amongst possibilities, how stupendous are the conceptions to which we are thus introduced! Suns (for what is true of our sun may be regarded as probable of others) vomiting forth cometic matter so violently as to communicate velocities capable of bearing such matter to the limits, or beyond the limits of the solar system : planets now passing through later stages of their existence, but presented to us, according to such theories, as once in a sun- 
like condition, and at that time capable of emulating the comet-expelling feats of the great central sun.

Are these thoughts too wild and fanciful to be entertained? They may appear so; yet where are we to find others less amazing? The comets of the warious orders-short-period, long-period, and nonperiodic-are there. Their existence has to be in some way accounted for; or if such explanation is at present impossible, as seems likely, we may yet follow the various lines of reasoning which present themselves. And we have very little choice. Take a comet of long period passing near the orbit, let us say, of Uranuseven as Tempel's comet, the parent of the November meteors, is known to do. Either that comet has been gathered in from outer space by the sun, and compelled to follow its present path by the disturbing influence of Uranus, or else-what? Only two other theories are available. Trace back the comet's path in imagination, round and round that oval path, which carries it across the paths of Uranus and the earth but nowhere else brings it within millions of miles of any possible disturbing influences. Rejecting the earth as insufficient in attractive might (or, at least, so inferior to Uranus as to leave us in no doubt in selecting between the two), we have only during the past of the comet, as so traced, the planet Uranus to which we can refer it. We have rejected the attractive influence of Uranus; but two other influences remain. Eruptive action in a former sun-like state, an action corresponding to the eruptive processes known to be taking place 
in the sun, is one possible origin. The mind of man, unapt though it is to deal with time-intervals so enormous as are required to transmute a giant orb from the sun-like to the planetary condition, may yet accept this interpretation, if no other present itself which is not still more appalling. Only one other, as it seems to me, remains, and this compels us to contemplate time intervals compared with which those required to change Uranus from sun to planet seem insignificant. If, as we are taught by the nebular hypothesis of the solar system, or, in fact, by any theory of its evolution whatever, the planet Uranus was once in a vaporous condition, extending as a mighty rotating disc far beyond its present sphere, and probably far beyond the path of its outermost satellite, we may conceive a comet arriving from outer space to be captured by the resistance of the once vaporous planet, not by its mere attractive force. But to what a result have we thus been led! If we accepted this view, rather than the theory that Uranus had expelled the comet, we should have first to carry our thoughts back almost to the very beginning of our solar system, and then to recognise, at that inconceivably distant epoch, comets travelling from sun to sun, and some of them coming from other suns towards ours, to be captured from time to time by the resistance of the vaporous masses out of which the planets of our system were one day to be evolved.

We do not know how the questions raised by such thoughts should be answered, although, as has been 
elsewhere shown, there is more evidence in favour of the theory of expulsion than of the other two theories just sketched. But we have reason to feel assured, as we contemplate a comet like that which now adorns our skies, that could we learn its history, a practical infinity of time would be brought before us as the aggregate of the time-intervals we should have to deal with. Nor is the marvel of the comet diminished by what we have learned from observation or from mathematical analysis. We have found that the tracks of comets are followed by countless millions of meteoric bodies, and thus the strangest thoughts - of infinity of space occupied by infinite numbers of cosmical bodies, aggregating towards multitudinous centres during infinity of time-are suggested to us. The telescope has shown us wonderful processes taking place during the comet's approach to the sun, and most wonderful process of all, the repulsion of the vaporous matter in the tail, as though to assure us that the expelling power of suns is even more than matched by the repelling power they exert on portions of cometic matter brought in certain conditions under their influence. Analysis by the spectroscope, that wonderful instrument which astronomy owes to Kirchhoff, has taught us much respecting cometic structure, showing that the light of the nucleus is that of a glowing solid or liquid (or of matter reflecting sunlight), the light of the coma that mainly of glowing vapour, while in the tail these two forms of light are combined. And polariscopic analysis speaks with equal clearness of the 
composite nature of cometic structure. But when all this has been said, we are little nearer to the solution of the mysterious problems which comets present to us. They still teach us, as they have so long taught, that 'there are more things in heaven and earth than are dreamed of in our philosophy.'

Spectator.

\section{THE AUGUST METEORS.}

ON the nights of August 10 to 14, and sometimes till several nights later, meteors or shooting stars, belonging to the family known as the Perseides, may be generally seen, at times showing in great numbers. Humboldt remarks that, on the festival of St. Lawrence (August 10), 'fiery tears' (supposed to be the saint's) fell from heaven, and he quotes Dr. Thomas Forster as saying that in a manuscript preserved in the library of Christ's College, Cambridge, written in the tenth century by a monk, the 10th of August is distinguished by the word 'Meteorodes.' Thomas Forster seems to have overlooked the effect of difference of style. In the seventeenth century, when the style was corrected, eleven days were dropped ${ }^{1}$ to set the calendar right; in

1 The day following September 2, 1752, was called September 14. In some popular books of astronomy, September 3 is substituted for September 2, and the singular statement made that calling the day following September 3 September 14 involved dropping eleven days. See Lockyer's Elements of Astronomy, and other such works. 
the tenth century the difference due to style amounted only to six days; so that the worthy monk's day for ' meteorodes' corresponded to August 16 of our time. The same meteor system may be meant, however, as perturbations may have affected the place where this family of meteoric bodies crosses the earth's orbit.

It is interesting to compare what was said about shooting stars by Humboldt, forty years ago, with our present knowledge. Then there were sensible men who saw, in the knowledge already gathered, the means of determining many points which writers in our time too often speak of as if they were recent discoveries. Then, also, there were representatives of the sterile school of science who ridiculed the results of sound reasoning, as if it were a fanciful hypothesis; men who called themselves observers, and spoke with enthusiasm of the making of observations, the collecting of observations, the classifying of observations, of everything, in fine, connected with observations, except the attempt to understand them and determine their value, which these cleverly practical persons called mere theorising.

Thus Olmsted at Newhaven, Conn., found that during the celebrated fall of shooting stars on the night between November 12 and 13, 1833, the fire balls and shooting stars all radiated from one and the same quarter of the heavens, namely, the vicinity of the star Gamma Leonis, and did not deviate from that point, though the star changed its apparent height and direction with regard to the compass points during the time of observa- 
tion. A curious observation-to be recorded, sent to some learned society, and forgotten? Not altogether so. Olmsted had a brain, as well as eyes wherewith to observe, and he reasoned that 'such an independence of the earth's rotation shows that the luminous body must have reached our atmosphere from without.' A fanciful theory, truly, said in effect the mere observers, for they proceeded to speak of meteors as though nothing of the kind had been shown. The fact is, however, they were to be excused: Olmsted's reasoning was demonstrative only for those who could follow him; he could give reasons, he could not give understanding.

So also, the occurrence of showers on particular days of the year-that is, at times when the earth is in particular parts of her orbit round the sun-was recognised by reasoning men as proof positive that meteor systems are extra-terrestrial. Humboldt, though he was not, properly speaking, an astronomer, yet studying astronomy like other sciences, with care to obtain an insight into the facts, saw the real meaning of this particular fact.

Now, of course, what was proved half a century ago by Olmsted, Encke, and others, is the accepted teaching of science. The August shooting stars gave the first obvious and easily understood evidence on the subject. When it was noticed that the larger comet of 1862 crossed the earth's track where the August meteors are encountered, every one could understand what Schiaparelli meant when he said that bodies following in the track of that comet would appear (as 
they passed through our atmosphere) to follow such paths as the August meteors traverse, i.e., paths seeming to radiate from the constellation Perseus. The beautiful reasoning of Olmsted had been neglected; the bold fancy of Schiaparelli was at once noted : not more noted afterwards, when by sound reasoning he showed that his lucky guess was a true one, than when he first threw out the idea. What followed is or should be well known. Adams, by an admirable mathematical investigation, showed that the November meteors travel in a period of $33 \frac{1}{4}$ years round the sun; and it was found that Tempel's Comet of 1866 traverses the self-same track. After which, it was justly regarded as proved that these meteor systems, following in the track of two comets, are in some way associated with them, and that, therefore, probably other systems of meteors are similarly associated with comets; and other comets, in like manner, followed by trains of meteoric bodies. ${ }^{1}$

The shooting stars, which are usually seen during a few nights in the second week of August, may be identified with the Perseids by observing whether the paths they follow-traced straight backwardspass through the constellation Perseus. The Star Map

1 A fact singularly misunderstood by many. Thus, in Mr. Dunman's charming Talks about Science, just issued, it is stated that, according to 'Messrs. Thompson' [Sir W. Thomson] 'and Tait,' the tail of a comet is ' a cloud of dust from the colliding masses' [of meteors] 'which the head trails behind it.' Mr. Tait said something of the sort-in fact, he wrote that a comet's tail is merely a shower of paring-stones; bat that was before he knew what astronomers had really proved. 
for August (see my Stars in their Seasons) shows that this constellation lies rather low down towards the north-east-a little northerly-at $\mathbf{9 . 4 5}$ on August 10, and at $9 \cdot 30$ on Augast 14. The constellation at those hours is moving ascendingly, and towards the east, remaining above the horizon all night. Meteors of the Perseid system may accordingly be seen at any time of the night: in greater number, but with shorter paths near Perseus; more scatteredly, but with longer paths at a distance from that constellation. When the moon is new on August 13, the opportunity for observing the August meteors is very favourable.

Scattered members of the August system may be seen for several days after the track of the main body has been crossed. But, as the direction in which the earth travels changes about one degree per day, science cannot admit that, as Mr. Denning opines (who has done excellent work in the observation of meteors), members of the August system can be recognised by still radiating from the proper 'radiant' of this system several weeks before and after the time of central passage. To say that a shooting star on September 14, for instance, radiated from the same centre as one seen on August 14, when the earth's course was thirty degrees different, is, for the mathematician, equivalent to saying, not that they belonged to the same, but that . they belonged to different meteor systems. 
Lovg experience has shown that men possessed with the gambling spirit (ninety out of a hundred if the truth were known) are not to be deterred from venturing small sums in order to win large fortunes, even by the clearest evidence that the price they have to pay is an unfair one. The Government lotteries in this country early put this matter to the test, in a way which possibly may have commended itself to the attention of the promoters of the Scotch lottery scheme. Having decided on a certain set of money prizes and a certain number of tickets, the Government did not offer the tickets to the public for more than they were worth, but for what they would fetch. They seldom failed to obtain from contractors at least 16l. for a ticket mathematically worth $10 l$. And the contractors not only showed by offering these sums their faith in human credulity, but practically proved the truth of their faith by disposing of their tickets for $5 l$. or $6 l$. more per ticket than they had paid Government for them. Thus the Government occupied a very favourable position. For every million they offered in prizes they received more than $1,600,000 l$. ; yet they asked no one to pay an unfair price. They left the contractors to do that, who were not only willing, but anxious to undertake the task of shearing the public. Nor were the public less ready to be plundered than the contractors were to plunder them. Government had to

III. 
protect the public, or rather tried to protect them, from the contractors, not by putting a limit to the price which contractors might obtain for tickets, but by endeavouring to prevent men of small means from buying tickets in shares of less than a certain value. of course, the laws made for this purpose were readily and systematically broken. The smallest sums were risked, and the only effect of the laws against such purchases was that higher prices had to be paid to cover the risk of detection. We learn that 'all the efforts of the police were ineffectual for the suppression of these illegal proceedings, and for many years a great and growing repugnance was manifested in Parliament to this method of raising any part of the public revenue. At length, in 1823, the last Act that was sanctioned by Parliament for the sale of lotterytickets contained provisions for putting down all private lotteries, and for rendering illegal the sale in this kingdom of all tickets or shares of tickets in any foreign lottery-which latter provision is to this day extensively evaded.' This was written forty years ago, but might have been written to-day.

The Scotch and West of England lottery schemes seem to have been carefully based on the experience obtained from those early lotteries. Men were willing in the last century to give more than $20 l$. to the contractors for tickets whose real mathematical value amounted only to $10 l$. It seems to have been assumed that in the nineteenth century the experiment might be safely repeated, only the promoters of the schemes 
did not fall into the mistake of allowing contractors to pocket half the profit. They proposed in Scotland to offer prizes worth three million pounds sterling for six million pounds worth of tickets; in the west of England they proposed to offer one million pounds worth of prizes for two million pounds worth of tickets. The balance of three millions in the one case and one million in the other would have been directly available for the purpose which the promoters of the schemes had in view; and everyone who bought a ticket in either lottery would be able to lay the flattering unction to his soul that, as he had given twice as much for the ticket as it was worth, one-half the price was a charitable offering.

It would probably, however, have served the purpose of the promoters of the scheme equally well, and would have exposed them, perhaps, to less direct animadversion, if they had adopted one of the Continental lottery systems. In these the holders of the lottery appear exposed to some slight risk of loss. The risk is exceedingly small. Practically, indeed, if they start with a sufficient capital, their risk is evanescent. Still, there is always a bare possibility that, instead of gaining millions, they may lose the thousands or tens of thousands with which they start. The holders of Continental lotteries may be more fairly charged with being 'mere gamblers' than our Scotch friends (who have been called so, but in reality by no means merit the name, since they ran no manner of risk); but the Continental systems have not been open to such 
effective assault from the mathematicians as the oldfashioned system, with set prizes and tickets selling for twice as much as they were worth. It is easy to show that with this system the holders of the lottery do in reality defraud the purchasers of tickets, and also (which with some has proved a more effective argument) that the greater the number of tickets any one purchases, the greater becomes the probability of his losing, until this probability becomes certainty. But it is not so easy to convince those who buy chances in a lottery on one of the Continenial systems that in the long run the holders of such a lottery gain as certainly on all ventures as the holders of an ordinary lottery, and even more quickly on the favourite ventures. Thus in the Geneva system, widely adopted on the Continent, the speculator may select any number from one to 90 ; five of these numbers are drawn at random; and if his number is among those drawn, he receives 15 times his stake. He ought to receive 18 times his stake. Thus he receives only five-sixths of the fair prize; and therefore, in the long run, the lotterykeeper would be practically certain to gain one-sixth of the total amourt ventured in this particular way. If the Scotch lottery had been so arranged as to allow only such ventures and $6,000,000 l$. were paid for chances, the lottery-keepers would win only about $1,000,000 l$., which seems small compared with the sum of $3,000,000 l$. which they hoped to obtain. But the selection of a single number is not by any means a favourite course on the part of speculators in Con- 
tinental lotteries. It is scarcely worth while to win 15 times a small stake, and few care to risk a large stake in this or any other venture. The public are always wanting to win thousands of pounds by risking only a few shillings. The Continental system allows them free indulgence in this taste. Instead of selecting a single number, the speculator can take two, three, or four numbers either in any order or in some selected order. In the French lotteries he was even allowed to take five numbers in a selected order; so that to win it was necessary that the five numbers drawn should be not only the five he had selected, but should come out in the precise order he had selected. In this case there was only one favourable drawing for the speculator out of $5,273,912,160,000$ possible drawings; so that theoretically, if he had ventured a halfpenny and chanced to win, he should have received rather more than $10,000,000 l$. (the exact sum should be 10,987,317l.). I have not heard whether any ventures of this tremendously speculative character were ever made; certainly none ever resulted fortunately for the speculator. But in any case the lottery-keeper would not have had to pay more than 5,000 million times the sum risked, for all he allowed (or rather promised) any one who might win on this venture was 10 million times his stake, or less than one 500th of what he was entitled to. Any sums ventured on this risk (called the quine déterminé) might be regarded as a present to the lottery-keeper; though it may be worth remarking that if a sufficient number ventured their money 
to give a fair chance of a fortunate drawing or of many such drawings, the lottery-keeper would win more on such ventures than on any others, despite the enormous sums he would have to pay in prizes. It is probable that many risked their sous and francs in the hope of winning a fortune on a quine déterminé; for unquestionably millions must have ventured on what to the uninitiated must appear scarcely less venturesome than the quine déterminé as respects the improbability of success-viz., the quine simple and the quaterne simple. Success in the former of these required that the five numbers drawn should be the five selected by the speculator, but in any order success in the second required that among the five numbers should be four selected by the speculator. On the average of an immense number of drawings in these ventures respectively, one venture in 43,949,268 would be successful among simple quines, and one in 704,880 among simple quaternes. Now, many speculators won in France on the latter venture, and even simple quine has several times been won. On one occasion it so happened that one and the same drawing lost the lottery-keepers on both these ventures. A speculator had selected the numbers $8,13,16,46$, and 64 , and another had selected for the same drawing $8,16,46$, and 64 . The numbers actually drawn were $8,46,16,64$, and 13 . Fortunately for the bank, the stakes were very small-in the former case $1 d$. and in the latter $2 \frac{1}{2} d$. The speculators won only 131,350f. and 20,852f. respectively. At their proper mathematical value in relation to the stake the prizes would have been $2,746,829 f$. and $176,220 f$. 
respectively; whence it will be judged how enormously the gambling public allowed themselves to be defrauded in return for the privilege of being allowed to risk their money on these more speculative ventures. The more speculative the venture, the greater was the deduction made from the just value of the prize. In the case of the simple drawing only $3 s .4 d$. per $1 l$. was deducted; for determinate drawing, $4 s .5 \frac{1}{4} d$. per $1 l$.; for simple ambe (two numbers), 6s. 6d.; for determinate ambe,

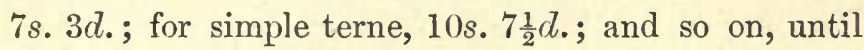
for simple quine so much was deducted that the speculator received only $6 d$. per $1 l$. in the earlier Geneva lotteries, and about $10 d$. in the French lotteries. Simple quine was not allowed in later lotteries on the Geneva system. The fact that the more speculative ventures were often successful proves to all who understand the laws of probability that immense sums must, on the whole, have been lost by gamblers who made such ventures. Those who brought themselves to think that in cases of great emergency-as the failure of the City of Glasgow and the West of England banks -it might be justifiable to take advantage of the gambling propensities of mankind would have done well to consider the evidence afforded by the success of the Continental system. They would probably have achieved their object more readily by adopting one of those systems; and they would, in appearance at least, have been freed from the imputation of winning money on a certainty, which even among gamblers is not considered to be strictly correct.

The Tines. 


\section{CONZUCT AND DUTI.}

In all ages of which any record has reached us, men of advanced mind (in their own age) have analysed the principles which regulate conduct, and have discussed those which should do so. In our own time the analysis of ethical considerations has been more fully developed than in any preceding age-partly, perhaps, because a wider experience is open to us, but chiefly because the progress of the doctrine of evolution has thrown new light on the subject. No one who has thoughtfully studied the doctrine of biological evolution can fail to see how important is its bearing on the principles which determine duty and should rule conduct. If the investigation of the laws of evolution in their merely scientific aspect has not shown this, the study of sociological works, such as those of Herbert Spencer, must have forced even those who do not accept the general doctrine of evolution to perceive that, among those who do, the doctrine cannot but have a most important ethical influence. For this reason, then, chiefly, the subject of the book before us largely occupies men's thoughts in our time.

Mr. Leslie Stephen has long been known as among the foremost and ablest of those who have dealt with the science of ethics during the last quarter of a century. His views on points of detail have been $\&$ Co.

The Science of Ethics, by Leslie Stephen. London: Smith, Elder, 
presented in essays which have appeared from time to time in the Fortnightly Review and elsewhere. In the present volume he has not collected such essays together, but has dealt with the general subject de novo; and he has here 'attempted to lay down an ethical doctrine in harmony with the doctrine of evolution, so widely accepted by modern men of science.' He remarks well that 'problems of this kind require to be discussed in every generation with a change of dialect, if not with a corresponding change of the first principles.' The standpoint from which he views the general subject differs from Mr. Spencer's. The great teacher of the modern doctrines of sociology has worked out an encyclopredic system, of which his principles of ethics are the outcome. Mr. Stephen, starting from the old ethical theories, has endeavoured to bring them into harmony with scientific principles, which he takes for granted. It is well that the subject should be examined from both points of view, that it may be seen how far the results coincide-as, of course, they should do, if the general principle underlying both lines of argument is sound.

I recommend this work to all who have examined the subject from Mr. Spencer's point of view, to all who prefer to examine it from Mr. Stephen's, and to all who, eschewing the modern doctrines of evolution, and decrying the supposed moral consequences of those doctrines, may wish to know what, according to scientific reasoning, these consequences are. 'These last, in particular, will be interested, we take it, to see how 
the moral laws which they have been in the habit of regarding as imparted from without, are regarded by science as springing from within the body social.

It wonld be impossible to describe in detail the various sections of this interesting work. Each chapter might well form the subject of a complete essay, or of a volume of discussion. The criterion which Mr. Stephen establishes in the progress of his work as the general principle of conduct is simply this, that a man is virtuous or the reverse, a worthy or an unworthy member of the community, in so far as he does or does not conform to the type defined by the healthy condition of the social organism of which he forms part. This must not be confounded with the doctrine, Soyez de votve siècle, for the characteristics of an age may be chiefly characteristics of disease, and conformity with them may, therefore, be not virtue, but the reverse. The type defined by the healthy condition of the body social, not that defined by its actual condition, is that with which conformity is desirable.

It will be seen that this doctrine limits conduct in two directions. As the good soldier neither lags behind nor goes unduly in advance of the main body (not even if he be the captain), so the man who best fulfils his duties to the social body of which he forms part, must neither fall short of the standard necessary for the healthy condition of the social organism, nor go unduly in advance. Take, for instance, such a quality as loyalty, in its ordinary but rather degraded sense. 
In former days it was essential to the social well-being that personal loyalty should amount to something like. devotion. And that quality, being essential to the very existence of a community, underwent development until, and even long after, this state of things ceased. It remains, then, a characteristic of many of the best men of a time when personal loyalty no longer has the specific value which it had in former times. Men who analyse the sentiment find, perhaps, in devotion to the King of Brentford, nothing specially virtuous; or, tracing his descent from these who first founded his family, they find small reason, perhaps, for regarding with respect the ground of the family's claim to loyal devotion. Men whose views of this particular duty have thus progressed, may be divided between two duties:- one, that of teaching those around them the avoidance of certain actions which appear to themselves derogatory to the dignity of manhood; the other, that of avoiding such offences as must arise from their not conforming to the type defined by the healthy condition of the social organism. They may do more harm by inculcating what they hold to be truth, and still more by doing what they consider theoretically just, than by conforming outwardly to the conditions of society as it exists around them. An officer, again, who ridicules that devotion to the colours which has led many a brave soldier to die rather than let the enemy take them, may in principle be very right, for a brave soldier is worth more than a blood-stained, shotriddled rag; and he who, in trying to save one, loses 
the other to his country or army, has not in that served his country well; but if that devotion, unreasonable though it is in principle, is absolutely essential to the efficiency of an army composed of not altogether well-reasoning elements, then the man who has ridiculed it, however reasonably, has done his country or its army an ill-service.

These cases are, of course, in themselves trivialthe reader will hardly need to be told that much more important matters are illustrated by them. If a citizen may be loyal to the community without any trace of loyalty to person or family; if a man may be a good and faithful soldier without the feeling (or having mastered as a weakness the feeling) of devotion to a standard or an ensign, so may there be many who are faithful to what they hold to be their duties without any of those feelings commonly spoken of as religious -though erroneously, for the word ' religion' applies equally in reality to any influence or principle restraining men's actions. Men who have learned that certain fears, hopes, and emotions are no more necessary to virtuous conduct than devotion to a standard is necessary to soldiers already devoted to a cause, must remember that this is not at present true of the social organism to which they belong. It is not only their duty, but the reverse of their duty, to ridicule feelings or beliefs essential to the well-being of the body social as it at present exists. The code we set up must be such as is good for the community, not that which is sufficient for ourselves. As our author well remarks, 
'we must admit that the ends which men pursue vary indefinitely, and that some men, possibly the mass of men, are fitted for those positions in the social organism which do not demand any great activity of the higher faculties, or make any strain upon a man's devotion to the race or to truth. ... When we speak of the morality of the lower type, we must mean that the habit of obedience to the moral law may be impressed upon it, although the moral instincts which make such obedience the spontaneous fruit of his character may be very imperfectly developed; and therefore, as a general rule, that to some extent other instincts, such as the fear of punishment (the hope of reward), the contempt (or the praise) of his fellows, have to be called into play, so as to make, as it were, a substitute for genuine morality.'

The wise moralist, on Mr. Stephen's theory, 'assigns no new motives; he accepts human nature as it is, and he tries to show how it may maintain and improve the advantages already acquired. His influence is little enough, but, such as it is, it depends upon the fact that a certain harmony has already come into existence, and that men are therefore so constituted that they desire a more thorough solution of existing discords. A sound moral system is desirable in order to give greater definiteness to aims and methods; and it is doubtless important to obtain one in a period of rapid decay of old systems. But it is happy for the world that moral progress has not to wait till an unimpeachable system of ethics has been elaborated.' 
VIVISECTION. ${ }^{1}$

ON March 28 last a meeting was held at the Royal College of Physicians, in London, attended by many of the most distinguished medical men of the day, the object being to 'bring the legitimate influence of the medical profession more effectually to bear on the promotion of those exact researches in physiology, pathology, and therapeutics, which are essential to sound progress in the healing art.' The Master of the Rolls remarked on that occasion, that 'it would be most desirable that the public should be informed upon the matters contemplated by the Association.' The book before us has been written in response to this wish.

It will probably be useless to commend the careful study of this little book (full of most interesting matter outside its special purpose) to the more sentimental of the anti-vivisectionists, for whom, by-the-way, Professor Owen adopts the title of Bestiarians. But for those anti-vivisectionists who are not prepared to allow a regard (very just in itself) for inferior animals to overrule all other feelings, the study of this treatise will be useful and instructive. They will see how much of the humanity of modern medical and surgical practice is due to the practice which the Bestiarians

1 Experimental Physiology-its Benefits to Mankind; with an Address on Unveiling the Statue of William Harvey at Folkestone. By Richard Owen, F.R.S., \&c. London: Longmans \& Co. 
describe as inhuman. Professor Owen shows that even inquiries, not necessarily directed at their outset to pain-preventing discoveries, have led to the relief of an enormous amount of human suffering and untimely death. As a striking example, consider the case of John Hunter's researches into the annual growth and shedding of the antlers of deer. A buck having been put at his disposal, Hunter placed a ligature around the carotid artery supplying the growing antler, on which the pulsations of the formative 'velvet' ceased, and the antler began to cool. The buck was released, and examined a week later. To Hunter's surprise the velvet had recovered its warmth, and the growth was proceeding as usual. The buck was thereupon killed (a 'fearful piece of cruelty,' though the annual hunting and killing of scores for venison is a 'noble sport'). He found the canal of the carotid had been obliterated; but sundry ordinary minute branches sent off below (i.e., between the heart and the ligature) had enlarged, had carried the blood to other capillaries communicating with the carotid above the ligature, and the enlargement of these previously inconspicuous vessels had restored the supply to the cold antler, and with it the power of growth. Take now the other side of the picture. Not long after, a coachman, suffering from popliteal aneurism (caused by the pressure of the hard margin of the box-seat on the vessels of the ham), lies at Hunter's (now St. George's) Hospital, awaiting amputation of the leg, to which operation he has given his consent. Hunter says to him, 'If you will let me 
make a small cut in your thigh, I may save your life and limb both.' 'God bless you, sir,' says the sufferer; 'do what you think best, so you put me soon out of this torment.' A ligature is thereupon put on the femoral artery, and immediately the tumour ceases to beat and begins to diminish. The patient exclaims that his agony is over. True, the leg begins to chill. Hunter will have no artificial heat applied, only flannels swathed round the foot and leg. In a day the natural warmth begins to return, but not the pulsations in the tumour. The morbid mass grows smaller and smaller, and in six weeks the coachman walked out of the hospital on both legs, cured of his aneurism. This was in 1793. In the years which have elapsed since, how many hundreds have been saved from torture and the risk of death by the application of this method, which substituted a comparatively trifling operation for rarely successful amputation. Even the operation is now usually avoided, pressure being substituted for ligature. And this is but one instance among many. Every one who wishes to gain a just and honest opinion on the vivisection question, should read this interesting work by our veteran naturalist. But those who wish to gain a cheap reputation for tender heartedness should avoid it, lest it convince them, against their will, that their tender mercies are very cruel.

Knowledge. 
The United States of America have had one great advantage over all the European nations. Experience which these nations have bought-and sometimes at a serious cost-the United States have gained (or had the means of gaining) at no cost at all. Nor have they usually been slow to avail themselves of this advantage. In their system of national education, in their manner of dealing with religious questions, in their mode of building their cities, and in a number of other matters on which national and municipal wellbeing depends, we recognise the influence of lessons taught by European history. But it would seem that there is no easy way by which the true laws of political economy can be learned. We find America, with all the experience of Europe, and especially of Great Britain, to guide her, falling into the same errors which led us astray; and when the effects which we recognise as the natural consequences of such errors are experienced, attributing them to any cause but the true one. The Times recently had occasion to refer to the sale of American meat in this country as illustrating the injurious effects of the American protective tariff. The vessels engaged, for instance, in bringing us American meat must, owing to that tariff, either return without cargo to America or return loaded with a profitless cargo. On this account the cost of bringing American meat here must necessarily be increased, and III. 
the profit to the producer diminished, while the consumer in England finds the difference of price between American and English meat less than it otherwise would be, by which the American producer still further suffers. I fear that the able reasoning of the Times in the article to which I refer will not have the effect on American political economists which, rightly apprehended, it should have. Many in America will find in the fact that ships carry full cargoes to this country, and return empty or loaded simply in ballast, clear evidence that the balance of trade is in favour of America. I had occasion during two visits to America to enter frequently into conversation with Americans interested in the question of Protection, and almost constantly I found their reasoning running in this faulty groove. 'On the one hand,' they argued, 'we protect our manufacturers, which is so much clear gain to us and loss to Europe (especially to the old country); and, on the other, our agriculturists lose nothing, for the trade in cereals, cattle, \&c., from the West has not diminished, but, on the contrary, has steadily grown.' The obvious answer to this reasoning seemed almost constantly to have been overlooked. European trade with the United States has unquestionably suffered by the American protective tariff, and probably (though this is by no means so certain) American manufacturers have profited even in the long run. But unquestionably agricultural interests in America have suffered, and as certainly the whole nation has suffered. The amount of produce from the Western States reaching 
Great Britain may-though even this is unlikely-have increased since the tariff was established, in the same degree in which it would have increased had free trade continued. But the value to the Western farmer of the price paid here for his produce has diminished at least one-half, simply because the price of manufactured goods, of labour-in short, the cost of living-has doubled in America, in consequence of the protective tariff. This increase in the cost of living might be taken as proving of itself the other point I have mentioned-namely, the injury to America as a whole. But there is a proof which may come home more directly to a great commercial nation-I mean the diminution of American maritime commerce. Twenty years ago, though Great Britain still stood before all other nations as respected the number and tonnage of vessels engaged in trade-that is, though we still possessed the largest share of the commerce of the world-the United States were rapidly gaining upon us, and it was clear that before long they would take the first place. Nor was this a matter to be regretted by us, or to cause anxiety or alarm. We should have gained almost as much as America herself by the natural growth of her commercial marine. In a few years, however, all this was altered. American maritime trade, instead of advancing more rapidly than ours, actually and rapidly retrograded; and though at the present time she is slowly advancing, we are advancing so much more rapidly that America is falling more and more in the rear, to her loss, and only in a 
less degree to ours. American political economists find the explanation of this in the effects of the war between the North and the South. Unfortunately it did so happen that the effects of the protective tariff, which alone were permanently injurious, coincided, in point of time, with the serious but transient effects of the war. It would have been easy for the clear vision of a soundly reasoning political economist to have distinguished between the two classes of effects; just as a skilful doctor can distinguish between the steady mischief wrought by some error of regimen and the transitory, though for a time more striking, mischief produced by an access of fever or by some bodily injury. But America possessed among her statesmen and politicians none who could thus recognise the true cause of the loss she was, and is, yearly undergoing. The history of European nations should have sufficed, however, to show that even in Europe, where the effects of war have always been more lasting by far than in America, the commerce of a great nation, however seriously it may suffer during war, recovers quickly when peace has been restored, a fact which should thus have convinced America that the mischief from which her commerce suffers is more deeply seated than any injury which the War of 1861-65 could have produced. One other error, which for many years misled us on this side of the Atlantic, America might also learn to avoid, would she but consider the lesson which we have learned at very heavy cost. I refer to the fond delusion that mischief to one country means gain 
to another. Long after Great Britain had rejected Protection, and accepted Free Trade in principle, the idea prevailed that, against any nation which continued to maintain a protective tariff, Protection was necessary in self-defence-the injury caused to us by their tariff being supposed to be compensated by injury caused to them by ours. That we have thoroughly learned the error of this view is best shown by the fact that we in no sort attempt to protect ourselves (save the mark !) against the free introduction of American agricultural produce into this country, as in old times we should have thought ourselves bound to do, as a compensation for the effects of their tariff on manufactured goods. We know from costly experience, that though by counter-Protection a nation can, indeed, injure another which maintains a prohibitory tariff, she injures herself in far greater degree. We have learned in fine, that Free Trade, like Mercy, is-

Twice bless'd-

It blesseth him that gives, and him that takes.

But although the principles upon which the more advanced Protectionists of America have advocated their cause are less directly interesting to us, it may be well briefly to consider them, because of the valuable lessons derivable from the tests which America has applied to those principles during the last few years.

The favourite argument with American Protectionists has been that a wide diversity of occupations is necessary for the social well-being of a nation, and that such diversity can only be secured, for a young 
and rising nation, by a Protective system. It will be remembered that this mode of reasoning was employed with considerable force, or rather vehemence, by Carey, of whom it has been truly said by Thorold Rogers, that ' he was the only economist of any reputation who maintained every fallacy in favour of Protection.' The argument is sometimes strengthened by combining the consideration of national greatness with that of social well-being. It is assumed that no nation can be truly great which has not a great variety of occupations. The assumption has arisen partly, perhaps, out of the observed fact that social well-being is certainly not favoured, but the reverse, by a tariff intended to encourage a diversity of employment. Compelled to admit the utter, though they think only the temporary, failure of Protection in this respect, Protectionists adopt the belief that-in some way not clearly shown - the greatness of a nation depends on the number and variety of employments in which its people are engaged. Both assumptions may be regarded as equally unwarranted, though neither one nor the other can be disproved by positive evidence, for this simple reason (of itself a fatal objection to the reasoning we are considering), that as a matter of fact variety of occupation naturally and invariably accompanies the growth of a nation. The fear of the Western farmers and the Southern planters of America that their country, unless saved from such a fate by Protection, would develope into a nation of farmers and cotton-growers, was an utterly vain one-we might almost speak of it as a 
ludicrous fear, were it not for the saving recollection of fears not less vain entertained in former times by ourselves, when as yet the laws of social and national well-being were misunderstood. If we were to admit the hypothesis, unproved and unsound though it is, that in the long run a nation will gain by an enforced diversity of occupations, we must yet reject the second assumption on which the reasoning we are considering depends. There is not merely a contradiction in terms, but a very real and actual contradiction, in the hypothesis that Protection can foster self-dependence. The manufacturers of all countries which, like America or our chief colonies, contain within their own bounds abundant resources of nearly all kinds, are naturally protected; for the manufacturers of other countries are not only weighted by the cost and risks of transport, but by market risks. Where, with these advantages in their favour, home manufactures are unable, in the long run, to maintain a successful contest against foreign manufactures, it may fairly be admitted that a nation's capital will be more advantageously employed in other pursuits. In such a case Protection can no more avail to encourage self-dependence than corks and bladders to make a powerful swimmer of one who is of weakly frame. Where, however, a new country is peculiarly fitted for some manufacture, it has been held open to question, by political economists of the highest order, whether Protection may not be advantageously used for a time, to encourage and develope a branch of industry which otherwise might not have 
been entered upon. In Mill's Principles of Political Economy, this argument has been urged, though cautiously. The Protection should be temporary, the industry suitable, the aid tentative. American writers have often referred to the passage (Book v. chap. x. sec. 1) as though it sufficiently supported, not merely all their arguments for the Protection of suitable industries, but also their arguments for the Protection of all undeveloped industries. It can scarcely be said that Mill's idea has hitherto been put to the test; because, as yet, every suitable industry to which Protection has been afforded for the purpose of development has struggled successfully to have such Protection extended far beyond the period of mere experiment. In the majority of cases, indeed, it may be urged that, the effects not having been hitherto so favourable as Mill supposed they might be, the experiment has not been tried long enough. But in the few cases in which a manufacture seems to have been encouraged by Protection, the experiment of discontinuing Protection has not been tried. In these cases, too, it remains altogether probable that the natural protection referred to above would in the same time have proved at least equally effective. In some cases an effect which might have been predicted has followed from a tariff practically prohibitory. For want of that healthy competition which would have compelled the exercise of all possible care and skill in some particular manufacture, articles of inferior quality continue to be manufactured. In such cases, instead of a branch of industry being 
developed, its growth and development have been checked by Protection.

But, after all, the great objection to these more subtle, but not less fallacious, arguments for Protection is that by which the arguments formerly used in this country have been overthrown-the test of experience. Americans and our own Colonists may maintain (though at the outset they did not very openly so argue) that material prosperity is far less important in their eyes than national self-dependence. Even then, indeed, they must admit that human interests are far more important than national interests; what is called national self-dependence being, in fact, only national self-interest under another name. But, apart from this, the question is one of degree, not merely of kind. The material prosperity of a nation may be, until the world is more ' thoroughly cosmopolitanised,' less important than the nation's self-dependence; while yet it may be a question what amount of material loss a nation may be prepared to undergo for some point more or less affecting national self-dependence. A man may regard the culture of his mind as more important than the culture of his body, and yet be unwilling to bring himself into ill-health by cultivating his mind to the undue expense of his body. Now, if an American considers the American tariff and its effects in this aspect, instituting a direct comparison between the good and the evil which it has wrought, and seems likely to produce hereafter, he would obtain some such result as this: "The agricultural and farm- 
ing interests of our nation have suffered enormously, and the whole nation, except a few small classes, has suffered seriously for many years; our mercantile marine is less already by millions of tons, probably by nearly one-half, than it would have been but for the tariff; foreigners have been encouraged by the high absolute profits of trade to come over here to make money, but the low relative value of money causes them to return with their gains to their own country; many wealthy Americans even have been tempted to go and to stay abroad simply because they find themselves far wealthier men abroad than at home; the frauds and peculations which always accompany a high tariff have long been rife among us, and there is too much reason to fear that the natural effects have extended to and are extending through political and commercial life; every day it is becoming more diffcult and dangerous to remove from our midst the very evil from which we are suffering; and many generations must pass before its effects, and the effects of its finally enforced removal cease to be experienced. But then, on the other hand, we have now learned to make our own clocks and watches. We have nearly learned to make our own paper and our own calico. With a little more time our ironmasters will no longer need (though it may not foliow that they will no longer ask for) a probibitory tariff; and the like with some other branches of manufacture.' Such seems to be a just comparison between the good and evil consequences to America of her Protective system. It is not for other 
nations to judge how such a comparison should affect Americans; but most Englishmen would have very little hesitation in deciding how it would affect us. The lesson is for us also a valuable one, though even we, who have not had to pay for the experiment one - withe of what it has cost the Americans, regard it nevertheless as too dearly bought.

Echo, 1877.

Note.-Since the above was written, I hare receired, among a number of American newspapers, the Baltimore Bulletin for February 3, 1877 , in which is a leading article comparing the recuperative energies shown by America and France. It opens as follows:-

'The United States is in the twelfth year after a great war. The pressure of the debt growing out of that war is felt by all the people to be heavier and hearier every day. Our industries are prostrate. There is no profit to be made out of any business. There seems to be no power of recuperation in the people. With the best soil in the worldwith a population more industrious, more vivacious, more enterprising than any other upon the face of the globe-we sit as if spell-bound by some fatal magic cbarm, incapable of making any use of any of our powers.

' Compare for a moment our present incapacity and inaction with the bounding recuperative energies which France has shown since the Germans left her soil in 1871. No nation was ever orerwhelmed by such total defeat and disaster as France in that war with Germany; no nation erer recorered so rapidly and so completely-in so sbort a time-as the French.' 
WE are apt to dwell somewhat too complacently on the good qualities of our nation, forgetting, or in some cases misapprehending, the origin of the race, and the presumable (even if it were not the observed) existence of other qualities. It is well we should remember that the English people is one of the oldest, strongest, and most obstinate breeds in the world. And what is the English people? The school text-books of history tell us that, in the main, it has descended from the Saxons, Angles, and Jutes, who conquered the country in the fifth and sixth centuries of the Christian era. A large admixture of Danes and Scandinavians must be admitted, seeing that once and again the AngloSaxons were beaten by Norwegian and Danish invaders, who, like themselves, made permanent settlement in the island. A strongly marked, but less widely reaching element was infused into the blood of the nation, according to these accounts, by the Norman Conquest, bringing French and Flemish blood, as well as that of the old sea warriors of the Northern Seas. Some of our best modern historians take this view of the nation. They show that the basis of our present society is Anglo-Saxon; that our language is in the main Teutonic. Norman and Norman-French have produced such effects on laws and on language as might be expected from the character of the Norman 
Conquest, and the interval of time which elapsed before the necessities of our Angevin kings compelled them to restore to the Anglo-Saxon element in the nation its original supremacy, social, religious, and linguistic.

According to this view we are a nation descended from conquering races. Our imperial instinct, which is, of course, a very fine thing now, though of old somewhat tainted with rapacity and brutality, has descended to us very naturally. And even if we would, even though we began in these later times to doubt whether the tendencies we inherit from brutal Saxon and fiery Norman are altogether the noblest qualities a great nation can possess, we should not be able to help ourselves. A nation can no more leap over its own shadow than a man can; and the old hopeless proverb says no man can do so, meaning that no man need hope to eradicate his innate evil tendencies. Let the few among us who have nobler and purer aspirations say what they will, a race sprung from the followers of Hengist and Horsa, Ella, Cerdic, and Cynric, a race strengthened by the alliances of Danish and Norman conquerors, must of necessity make a fighting people. Trade and its developments may occupy us for a while. But from time to time we must engage in the pastime of our ancestors. "War was the Englishman's " shield play" and "sword game," says Green ; 'the gleeman's verse took fresh fire as he sang of the rush of the host and the crash of its shieldline; their arms and weapons, helmet and mailshirt, tall spear and javelin, sword and 
seax, the short, broad dagger that hung at each warrior's girdle, gathered to them much of the legend and the art which gave colour and poetry to the life of Englishmen'-with much more very pretty writing about the charms of war and piracy to our Saxon ancestors.

To many it may not be pleasant to contemplate the other side of the shield. The Jingo breathing blood and fury, must find it pleasanter to be told he comes from a furious and bloodthirsty ancestry, than to learn that far more probably he comes of a breed whichbrave and fierce though it may have been-was forced for many generations to bend the neck to conquerors. I am not sure that the few generations during which the Saxons and Danes had to yield to Norman chiefs did not considerably diminish the warlike ardour of those races, even though in the long run Saxons and Danes showed themselves the more tenacious and enduring races. But, unless probabilities in the first place, and the strongest possible form of existent evidence in the second, are wholly deceptive, we are not in the main an Anglo-Saxon race at all. The story which tells how the British inhabitants of the country were driven into Cornwall and Wales, except some few who crossed over to Brittany, and other few who took refuge in Cumberland, has never had any historical support of value, and is a preposterous tale on the face of it. No nation was ever so driven out from such a country as Britain then was. Historians who recognise the utter unlikelihood of the event, and 
the lying capabilities of the one historian whom they trust, can only explain the eradication of the Britons by supposing, a stubbornness in that people quite inconsistent with the feebleness and effeminacy alleged as the reason of the appeal to Hengist. Such historians seem to imagine that a stubborn, well-armed people, largely surpassing their invaders in number, could as easily be swept into the western corners of the island as one might sponge out the colours of a twelve-inch map. All the evidence which history gives-or rather suggests-is overlooked, because the ancient language gave place to the Anglo-Saxon ; as if it were not easier to suppose that the Saxons forced the conquered to speak their language than to suppose that they'were so stupid as to kill or drive out the people who made the chief value of their conquest. But we need not consider probabilities. In these days there are two great tests of race-one of high value, the other unmistakable. If they had given concordant evidence in this case, I suppose we should not have been misled even by that mistaken patriotism which makes some historians anxious to forget the true ancestry of their people when that ancestry is not to their taste, much as a patrician might foolishly prefer to blot out from the family genealogy the record of an ancestor's marriage with a 'lass of low degree,' even though some of the best qualities in his own blood and temper might have been derived from that despised source. Unfortunately the two forms of evidence point different ways. By the test of language we should be judged 
assuredly to be Saxon, though some Welsh writers have laboured hard to prove that the English language contains a large mixture of Welsh words. The test of language is not infallible, however. Indeed, if we may judge from what some writers alleged in the thirteenth and fourteenth centuries, we owe it mainly to accident that we do not now speak a language more French than English. But the evidence derived from bodily and mental characteristics is infallible when it speaks plainly. And assuredly it speaks plainly in this case. In England we occasionally meet with persons of the yellow-haired, fair-complexioned, blue-eyed Saxon type, and perhaps as often with persons of the lighter complexioned Scandinavian type. But the people, as a whole, is a dark-haired and dark-eyed people. Even in the North-east, where the Scandinavian type is often met with, and in the East and South-east, where the Anglo-Saxon type is commonest, either type is seldom seen compared with the dark British type. In Cumberland, Wales, and Cornwall we find, of course, purer British races, not the same race, which of itself affords a proof that the old British inhabitants were not driven into these parts of the island. But let anyone walk or ride through London, examining West-end and City regions, poor neighbourhoods and rich, and compare the number of dark and fair-complexioned persons he sees. He will perceive how decisive is the evidence of the parentage of our people, let their institutions be what they may, or their language as Saxon as even Freeman or Stubbs could desire to prove 
it. We learn the same from a method of inquiry which cannot be quite so easily followed. The average shape of our English heads proves that we are more Celtic in blood than we are in laws and language. Unless I mistake, some of the best and some of the worst qualities of the English nation attest the British onigin of the race, regarded as a whole. And though the men who have held the foremost place in British history (I am very far from meaning our kings or queens) have for the most part had less British than Norman and Saxon blood in their veins, yet I doubt whether there is one man in a thousand here wha does not share, in greater or less degree, the blood of the British tribes which the Romans found in this island nineteen centuries ago. In this sense, it has been correctly said of the English people that, 'while receiving successive admixtures and undergoing developments, it has remained for centuries substantially the same; and, what is to be well borne in mind, it has had more effect on its conquerors, masters, legislators, gentry, and clergy, than they have ever had upon it.' 


\section{A LATE BOAT RACE.}

THE sports in which our youth take delight are of little use unless they encourage manliness and generosity, not only in those who take part in them, but in those also who witness and discuss them. I regret to notice sometimes comments on the University Boat Race which seem at once ungenerous and unsportsmanlike; they also betray considerable ignorance of the actual conditions under which contests of the kind are undertaken. In the Spectator a writer does not scruple to insinuate that the Cambridge crew of 1878 were not only beaten, but disgraced. 'It is usual to say,' he writes, ' in the case of a race like this, that the losing crew were beaten but not disgraced; we have no wish to depart from the polite phraseology which usage sanctions, but it is obvious that there must have been a screw loose somewhere. When the raw material which the captains can draw from is practically identical, a repetition of the Harvard fiasco ought not to be possible in a race between Oxford and Cambridge.' Among the faults committed by Cambridge, it seems, was her attempt to secure the lead at starting, which this writer considers ' simply suicidal.' But in this he confounds the tactics of boat racing and horse racing. Forcing the pace at starting is unquestionably disadvantageous for the weaker horse in a race; but getting the lead at starting in a boat race is a positive 
advantage, as everyone knows who has ever rowed astern of a racing-boat. No boat's chance has ever been injured by forcing the pace, and in nine races out of ten the boat which has been first for the first halfmile has been first past the winning-post. What he adds about the Cambridge boat being 'done with' and 'done for' at Hammersmith, and about a rumour reaching Putney that the Cambridge boat had been taken off at Chiswick, is simply absurd. The writer of the article may have been at Putney while the race was rowed; but he should not have believed that so preposterous a rumour was ever mentioned there, except as a joke. It is only necessary to examine the time-records of the latter half of the race to see that Cambridge were not 'done for and done with' at Hammersmith, nor yet at Barnes Bridge. The race was over at Hammersmith, beyond all doubt. That is, it was clear then, and even earlier, that, bar accidents, Oxford must win. But Cambridge rowed the race out to the end. They not only did not fall astern faster in the last half than in the first half of the race, but they made as good time, mile for mile, in the latter half as in the first.

To say the truth, many who are either unfamiliar with the conditions under which boat races are rowed, or do not duly consider them, misapprehend altogether the probabilities in the case of a struggle of this sort. They seem to think a close race should be the usual event, instead of the reverse; and when one boat wins by a long distance they think it necessarily follows that 
the other had some gross fault of style, or committed: some error in training, or had a screw loose somewhere; whereas, as likely as not, they were simply beaten because they had to contend against a stronger set of men.

Now I do not assert that the Cambridge style was. perfection in the late race, or that the men were not overtrained. On the contrary, I think their style was slightly inferior to that of Oxford on the whole (it was certainly superior in one or two respects, as everyone whose opinion was worth anything admitted); and it réquired only a glance at the two crews on the morning of the race to perceive that Cambridge were overtrained. To use an old-fashioned way of speaking about training, the Oxford men had trained red, while the Cambridge men had (mostly) trained yellow-which last, by the way, was at one time thought, absurdly enough, the perfection of training. But it was well known, long before either crew was selected, that Cambridge would have a weaker erew this year than Oxford; and when selection was finally made the difference in this respect was notorious. It is not too much to say that the most perfect coaching and training would not have enabled Cambridge to make a close race of it this year. Now the point to which $I$ would call attention is this :Given two sets of eight men, each chosen from a large number, to contend in any feat whatever, whether of strength, skill, or endurance, the chances are against a close approach to equality in the aggregate effectiveness (which is, in fact, the average effectiveness) of the 
two sets of men. If the best broad-jumpers of Oxford and Cambridge were to contend, the average range of one set would, in all probability, differ several inches from the average range of the other set, nor would any amount of practice or training alter the result. So in any kind of contest whatever, as the records of such contests show. Suppose now the Oxford eight in the late race to have been so far superior in strength to the Cambridge eight that they were able to propel their boat one foot farther per stroke than the latter, after an equal amount of practice, with equal coaching, equal style, and with like equal conditions as to health of the men, qualities of the boat, and so forth. This, surely, will not be considered an unlikely difference in any case, and certainly not in the actual case, considering what was known, even as far back as last November, respecting the performances of the individual members of both crews. The distance which a racing-boat is propelled in a single stroke is about twenty-eight feet. Of course the actual distance would be greater if one stroke only were taken; but I am referring to the distance due to each stroke in a race. Thus we may allow for each stroke half a boat's length; and in sixty strokes, under equal conditions, Cambridge would lose one boat's length; and as Cambridge rowed about nine hundred strokes in the late race, she would, according to this estimate, have been fifteen lengths behind Oxford at the finish. She actually lost by about ten lengths, of which nearly four were lost before she reached Hammersmith Bridge. Cambridge, in fact, 
actually lost at the rate of about eight inches per stroke. This result is not much affected by the more rapid stroke rowed by Cambridge during part of the race. Changing the rate of stroke is rather changing the way of putting in the work than changing the rate of working. Yet it may fairly be admitted that Oxford could have won by several more lengths had they pleased; or, rather, could have reached the winning post several seconds earlier had they been hard pressed. But probably the best Oxford could have done would have been equivalent only to a gain of about a foot per stroke; and the reputation of the Oxford crew, individually and collectively, justifies the belief that it was 'in them' to beat Cambridge at that rate per stroke, whatever the style adopted by Cambridge and whatever the system of training. If this were so, as may reasonably be believed, Cambridge did all that was in them to do, as well in practice and in training as in the race itself, from the first stroke to the last. Men can do no more than their best. It is of races so rowed that 'it is usual to say that the losing crew were beaten but not disgraced,' and this could fairly be said of the race of 1878. Men who were able to watch the race closely admit that there was no sign of flinching in the Cambridge boat from first to last, though there were signs enough of distress, as there always will be in a long stern chase.

Echo, 1878. 


\section{ARE WE JEWS?}

IN his Study of Sociology, Herbert Spencer imagines 'an independent observer, living in the far future,' speculating in perplexity concerning the Judaisms of the religion of our time and country. 'Yet,' says this future observer, 'though the English were greatly given to missionary enterprises of all kinds, and though I sought diligently among the records of these, I could find no trace of a society for converting the English people from Judaism to Christianity!' This future observer remarks with surprise on the rigid observance of every seventh day, "which had been deliberately discountenanced by the Founder of the Christian religion.' But such utterly rigid Sabbatarianism, such inflexible woodenness of observance, as the inhabitants of St. Kilda, headed by their minister, have recently displayed, for the edification of the adjacent islands of Great Britain and Ireland, is not supposed to have come within the ken of the future student of our times. On the 12th the Government gunboat 'Flirt' arrived off St. Kilda with seed, corn, and provisions for the islanders; and the commander, regarding the anchorage as unsafe, wished to land the provisions at once; but the St. Kildians refused to render any assistance, because the labour might not be concluded by midnight, and the next day was the Sunday. They expressed their willingness to trust in Providence that 
the weather would continue favourable until Monday, and also, it is to be assumed, that the captain of the 'Flirt' would be content to wait till then. Fortunately this turned out to be the case, and the inhabitants did not suffer from the rigidity of their Sabbatarianism. It must be admitted that this instance of the strict observance of the old Jewish law has been surpassed in absurdity, and that, too, within times recent enough. Folks have been punished in Scotland for saving the lives of animals on Sunday; and warned not to offend again, lest punishment should befall them, for saving the lives of a shipwrecked crew. In one case such a crew was suffered to perish that the Sabbath might not be broken. But it was supposed that this barbarism had passed away in great part. It is, however, a far cry to wild St. Kilda. We must assume that the minister who headed (save the mark !) the St. Kildians in this matter was either native to the isle, or received his religious training half a century ago in some Scottish seminary where Sabbatarianism of the old type was rampant. Yet we cannot but recall how, quite recently, a minister in a Scottish region not quite so wild and desolate as St. Kilda rebuked his people for reaping on Sunday, and the weight of the Queen's opinion had to be quoted against his before they could be encouraged to continue the practice. Although one does not exactly know how the Queen came to be an authority on such questions, she certainly showed much more judgment than the minister in this instance. $\mathrm{He}$ and his reverend brother at 
St. Kilda would probably have been scandalised-had it pleased Providence to have brought them into the world nineteen centuries or so ago-by the language of a certain Jewish Teacher, who asked, 'What man shall there be among you that shall have one sheep, and if it fall into a pit on the Sabbath-day, will he not lay hold of it and lift it out?' adding, presently, 'Wherefore, it is lawful to do well on the Sabbath-day.' They would have reasoned that the owner of the sheep ought to trust in Providence, which would provide for the creature's well-being the next day. Even the Pharisees, who were not nearly so strict as the St. Kildians, argued the matter somewhat in that way: "There are six days in which men ought to work; in them, therefore, come and be healed, and not on the Sabbath-day.' These were men of whom the Teacher said that ' each one of them on the Sabbath loosed his ox or his ass from the stall, and led him away to watering'; so that they were not utterly devoid of reason.

But while we thus wonder at St. Kildian idiocy, let us not be altogether confident that we may not become a by-word for similar stupidity ourselves. It seems scarcely credible, but it is the fact, that the weather warnings which have been sent daily from our Meteorological Office to the Continent during the winter months have been discontinued for the summer, so far as Sundays are concerned. The Continental press naturally make fun (somewhat scornful and bitter fun, though) of this regulation. They express a fear lest storms may not prove strictly Sabbatarian, even in summer- 
time. Considering that the weather warnings relate, not indirectly, to the saving of life and property, it does seem as though, apart from the practical absurdity of the regulation, there was something specially unchristian about it. For though Christ, in his teaching respecting the Sabbath, made no direct reference to telegraphic communications respecting the weather, He showed clearly enough what He would have said about them had the Pharisees raised the question. If 'one sheep,' He said, 'fall into a pit, will ye not lay hold on it, and lift it out?' 'How much is a man better than a sheep?' He continued. Obviously $\mathrm{He}$ would have extended the argument had occasion arisen by asking, 'How much is the crew of a ship more worth than one man? And how much are many endangered crews more worth than one crew?'

Nature, indeed, as it seems to me, answers for us all questions concerning the seventh day's rest. Sabbatarians lay great stress on the assumed fact that the rest is found good for body and brain-a fact which, if proved, would mean little more than that long-continued habit has made such rest a necessity. But they pay little attention to the fact that nature knows no seventh day's rest. The earth does not pause in her orbital motion round the sun, nor the moon on her motion round the earth. The tides and currents of the ocean continue their motion, and the waves rest no more on the Sabbath than on week-days. Winds blow and rains fall on that day as on the rest. All forms of life, vegetable and animal, continue un- 
affected, save only as they are related to man in those countries where the seventh day's rest is observed. There is sound logic in the story of the manna and quails in the desert. 'How long refuse ye to keep the Lord's commandments? See, for that the Lord hath given you the Sabbath, therefore $\mathrm{He}$ giveth you on the sixth day the bread of two days.' This was soundly reasoned by Moses, who, being learned in all the wisdom of the Egyptians, probably understood something of logic. We cannot wonder that in the sequel the people recognised the justice of his reasoning. 'So,' says the story, ' the people rested on the seventh day.' Now, if our nineteenth-century storms would only observe the seventh day as carefully as did the manna and quails in the wilderness, the change recently made by our Meteorological Office would be reasonable and proper. Unfortunately they do not, as we have had reason to know ever since the change was made. If we must in such matters follow the example of the Jews of old, our meteorologists should not hesitate ta revert to their winter rules. The Sabbatarians of the days of Antiochus Epiphanes were strict enough in all conscience, yet, when they had waited in vain for a Sabbath miracle to save them, they very sensibly gave up a too rigid adherence to the law of Moses. The story is a sad one, but instructive; and if it were permitted to question any of the arrangements of our Church by law established, one would be tempted to suggest that the story might be read once a year, instead, let us say, of the history of Bel and the Dragon, 
or of Susannah, or of Tobit and his Faithful Dog, or other apocryphal narratives; for the Books of Maccabees are good history. When the soldiers of Antiochus attacked, on the Sabbath, those who had fled to the wilderness, these would neither fight nor hide, because to do so they must have lifted stones. They were accordingly slain with their wives and children to the number of a thousand. On this the rest said, every man to his neighbour, 'If we shall all do as our brethren have done, and not fight against the heathens for our lives, they will quickly root us out of the earth.' So they determined, very wisely, to change their tactics, saying, "Whosoever shall come up against us to fight on the Sabbath-day, we will fight against him.' Pursuing this plan, 'they slew the sinners in their wrath, and the wicked in their indignation.' Our meteorologists would do well to follow the example of Matthias and his friends. They have tried the experiment of omitting Sunday storm-warnings, but Sunday storms have not ceased. Every meteorologist should, therefore, say to his neighbour, 'Whatsoever winds shall threaten to blow on the Sabbath-day, we will telegraph news of the same to the nations round about.' After all, though we are unquestionably in need of the antiJudaic missionaries suggested by Herbert Spencer, we call ourselves Christians, and if the Founder of Christianity ' made clay' on the Sabbath-day, and that, too, when $\mathrm{He}$ might, as Christians believe, have restored the blind to sight with a word (or on the morrow), our Meteorological Office might not be un- 
willing to devote a few minutes on Sundays to the transmission of weather warnings, when needed, whereby, perchance, many lives and much property might be saved from destruction.

PARADOXES AND PARADOXISTS. ${ }^{1}$

Iv Professor De Morgan's Budget of Paradoxes the reader will find how a master of logic and mathematics dealt with error, and in what spirit the paradoxists received correction at his hands.

We must notice, however, that by the word paradox De Morgan does not understand in all cases mere error. On the contrary, some of the paradoxes he here deals with are now recognised as either probable or admitted truths, and were only paradoxes when they were first enunciated. The Copernican theory of the solar system is only not included among the paradoxes 'because everybody knows' it. A paradox is, in fact, any opinion or theory which differs from general opinion either in subject-matter, method, or conclusion. Consequently a paradox may be just, for general opinion may be erroneous. In precise proportion, however, to the probability that general opinion is correct is the probability that a paradox is erroneous. It is other-

1 A Budget of Paradoxes. By Augustus do Morgan, F.R.A.S. and C.P.S. of Trinity College, Cambridge, London: Longmans \& $\mathrm{C}$. 
wise with heterodoxy, which relates to instances where general opinion is divided, and a majority only (whether of number or power) favours the orthodox view. In science, however, paradoxy is more often met with than heterodoxy; and as general opinions are not now formed in science without strong evidence, paradoxy is, as a rule, error, only distinguished from other error by being blatant.

It may be asked, however-and is a very suitable inquiry at the outset-how we are to assure ourselves that the paradoxist may not be a great discoverer. If the present theory of the universe was called three centuries ago the ' paradox of Copernicus,' how can we be sure that the paradox of Reddie, or 'Parallax,' or Morrison, may not be the future theory of the universe? The rule is very simple, and is clearly enunciated by De Morgan. ' New knowledge,' he says, 'when to any purpose, must come by contemplation of old knowledge, in every matter which concerns thought (mechanical contrivance sometimes, not very often, escapes this rule). All the men who are now called discoverers, in every matter ruled by thought, have been men versed in the minds of their predecessors, and learned in what had been done before them.' (The italics are mine.) 'There is not one exception. ... If any one will undertake to show a person of little or no knowledge who has established himself in a great matter of pure thought, let him bring forward his man and we shall see. This is the true way of putting off those who plague others with their great 
discoveries. The first demand should be, Mr. Moses, before I allow you to lead me over the Red Sea, I must have you show that you are learned in all the wisdom of the Egyptians upon your own subject. The plea that it is unlikely that this or that unknown person should succeed where Newton, \&c., have failed, or should show Newton, \&c., to be wrong, is utterly null and void. It was worthily versified by Sylvanus Morgan (the great herald, who, in his Sphere of Gentry, gave coat-armour to "Gentleman Jesus," as he said), who sang of Copernicus as follows (1652):-

\author{
If Tellus winged be, \\ The earth a motion round; \\ Then much deceived are they \\ Who nere before it found. \\ Solomon was the wisest, \\ His wit nere this attained; \\ Cease, then, Copernicus, \\ Thy hypotheses vain.
}

Newton, \&c., were once unknown; but they made themselves known by what they knew and then brought forward what they could do; which I see is as good verse as that of Herald Sylvanus. The demand for previous knowledge disposes of twenty-nine cases out of thirty, and the thirtieth is worth listening to.'

The advantage of this rule is that it is so easily applied. It should be useful to the paradoxists themselves, though that, perhaps, is too much to hope for. But in any case its reasonableness and simplicity should commend it to those who are addressed by the paradoxists - to that too wide general public, which 
cannot for its life tell whether the paradoxist is a great discoverer or an egregious blunderer. When a paradoxist comes before the world with such statements as generally accompany paradoxy, when he boasts that, without knowing this or that, anything of logic or much of mathematics, he has yet been able to show all the philosophers to be mistaken, then this rule at once disposes of the paradoxist's claim. It is a thing which has never happened for a man without wide and thorough knowledge of a branch of science to make great discoveries in that particular branch; therefore, the public is not bound to entertain the possibility that the thing has at length happened; the assertion may be dismissed as incredible. It would be well if this principle were generally known and acted upon; not well in the interests of the general public, who gain by being puzzled and set a-thinking, but well in the interests of those who desire to spread exact knowledge, and who at present find their labour doubled through the perplexities engendered by the paradoxists.

Were it not for this necessity of removing the perplexities of the less instructed, the student of science who hears a paradox propounded might act simply as Sir J. Herschel is said to have done when a paradoxist asserted that he had squared the circle. 'Then,' said Herschel, according to the story, 'I wish you a very good morning.'

That De Morgan partly intended to be of use to those whom paradoxists generally succeed in perplex- 
ing can scarcely be questioned, though, as it appears to us, his main object in preparing the budget was to collect together the curiosities of paradoxical literature. 'My intention in publishing this budget in the Athenoum,' he says, 'was to enable those who have been puzzled by one or two discoveries to see how they look in the lump.' But he often gives page after page not apparently penned in fulfilment of this intention. Accordingly, the book is full of matter of very varied interest. There is much to amuse even the general reader, who may care nothing whatever about the true or false in science; much to interest the dabbler in science, the natural prey of the blatant paradoxist; but there is also much to engross the attention of the student of science.

The paradoxes chiefly treated of in this book are(1) those relating to the solar system, gravitation, \&c.; (2) the quadration of the circle and the attempted solution of other famous mathematical problems long recognised as insoluble; and (3) the 'number of the beast.' But many other paradoxical subjects are dealt with at less length.

Among the paradoxists who have invented new systems of astronomy, our friend 'Parallax' gets a page or two, though he was not so well known when De Morgan wrote as he has since become. It appears he went in $\mathbf{1 8 4 9}$ by the name of S. Goulden. Later, as we know, he has been called 'Rowbotham'; though the name in which he specially delights has been always 'Parallax.' This name may have been chosen 


\section{LIGHT SCIENCE FOR LEISURE HOURS.}

to indicate his wish to change the accepted views of the universe, for does not Liddell and Scott's Lexicon give, as the meaning of the verb $\pi a \rho a \lambda \lambda a ́ \sigma \sigma \omega$, 'to change or alter a little, esp. for the worse,' or else, as indicative of the ingenuity of the man in avoiding any argument which appeared irresistible (for the verb means also to slip aside, escape, or dodge). His skill in this respect was remarkable. He would reason at any length with those who had a smattering of astronomical knowledge, and he would only occasionally, by some reference to Scripture, exchange what resembled argument for an 'appeal to the gallery;' but when a really unanswerable argument was brought against him, he had a simple but effective formula, which always afforded a loophole for escape. ' Come, sir,' he would reply, 'you have said your say; others wish to speak as well as you.' It is probable that De Morgan had very little conception of the real position of this worthy, whom he regarded as an ingenious simpleton; but his remarks are worth quoting, especially as they give a very good idea of De Morgan's style :-

'August 28, 1865.-The Zetetic Astronomy has come into my hands. When, in 1851, I went to see the Great Exhibition, I heard an organ played by a performer who seemed very desirous to exhibit one particular stop. "What do you think of that stop?"I was asked. "That depends on the name of it," said I. "Oh! what can the name have to do with the sound? That which we call a rose," \&c. "The name has everything to do with it; if it be a flute-stop, I think 
it very harsh; but if it be a railway-whistle stop, I think it very sweet!" So as to this book : if it be childish, it is clever; if it be mannish, it is unusually foolish. The flat earth floating tremulously on the sea, the sun moving always over the flat, giving day when near enough, and night when too far off; the selfluminous moon with a semi-transparent invisible moon, created to give her an eclipse now and then; the new law of perspective by which the vanishing of the hull before the masts, usually thought to prove the earth globular, really proves it flat; all these and other things are well fitted to form exercises for a person who is learning the elements of astronomy. The manner in which the sun dips into the sea, especially in tropical climates, upsets the whole. Mungo Park, I think, gives an African hypothesis, which explains phenomena better than this. The sun dips into the western ocean, and the people there cut him in pieces, fry him in a pan, and then join him together again, take him round the underway and set him up in the east. I hope this book will be read, and that many will be puzzled by it; for there are many whose notions of astronomy deserve no better fate. There is no subject on which there is so little accurate conception as that of the motions of the hearenly bodies. The author, though confident in the extreme, neither impeaches the honesty of those whose opinions be assails, nor allots them any future inconvenience; in these points he is worthy to live on a globe, and to revolve in twenty-four hours, 
The next paragraph relates to a disciple of 'Parallax '- Common Sense'-and his work, Theoretical Astronomy Examined and Exposed. 'I hope,' says De Morgan, 'it will not be the last.' He could not, however, have foreseen how poor Mr. Hampden would suffer by the study of ' Parallax' and ' Common Sense.' In fact, we venture to question the theory that good is to be gained by the study of the ideas of paradoxists. A student of science may with advantage turn over the leaves of a work written by an honest paradoxist; because he can thus find what mistakes the denser readers of science-treatises are likely to make. He may use his paradoxist, in fact, as a foolometer. But paradox books are not safe reading for the general reader. They are not, perhaps, quite so misleading as text-books of accepted science written by ill-informed; persons-simply because the general reader knows beforehand that the paradoxist is a nonsense-monger, and so accepts none of his statements, whereas he supposes that a treatise of orthodox science is sound throughout. No one is likely to believe that there is a semi-transparent moon merely because 'Parallax' says there is; while probably nine-tenths of those who have read Lockyer's Elementary Lessons in Astronomy are satisfied that seamen find local time 'by observing when the sun is at the highest point of its path' (article 564); that when the sun moves fastest the dial will get before the clock, and vice versâ (article 416); and so on with the other blunders in that too clever compilation. But though the mischief 
done by the paradoxist with his false science is less serious than that accomplished by those who write about real science without sufficient knowledge, yet the study of paradoxes is not on that account to be recommended.

The subject of circle-squaring occupies a large part of the present work; perhaps, on the whole, there is too inuch about it. A similar remark applies to the ' number of the beast,' on which, however, De Morgan quotes some amusing stories. Here is one, taken from Dr. Thorn (who claimed, by the way, to have the number of the beast in his own name):- 'A Mr. James Dunlop was popping at the Papists with a 666-rifled gun, when Dr. Chalmers quietly said, "Why, Dunlop, you bear it yourself," and handed him a paper on which the numerals in

$$
\begin{aligned}
& \text { I A C O B V S D V N L O P V S } \\
& \begin{array}{llllllll}
1 & 100 & 5 & 5005 & 50 & 5
\end{array}
\end{aligned}
$$

are added up. This is almost as good as the Filii Dei Vicarius, the numeral letters of which also make 666 .' Again, 'All the diviners, when they get a colleague or an opponent, at once proceed to reckon him up; but some do it in play and some in earnest. Mr. David Thorn found a gentleman of the name of St. Claire busy at the beast number; he forthwith added the letters in $\sigma \tau \kappa \lambda a \iota \rho \varepsilon$ and found 666 ; this was good fun. But my spiritual tutelary, when he found he could not make a beast of ine' (Dr. Thorn had tried this exercise), 'referred the difficulty to the Almighty; this was poor 
earnest.' I venture, by the way, to offer a solution of the mystery, which will doubtless be at once accepted. Observe that 6.66 shillings amount appreciably to $6 s .8 d$., a fee which may be called the Devil's Own. The Devil's Own Fee expressed in the decimal system (which is itself Antichrist, according to some), presents very naturally the number of the beast.

The following will interest those who have read certain controversies about perspective in the correspondence columns of the English Mechanic:-'Mr. Parsey was an artist who made himself conspicuous by a new view of perspective. Seeing that the sides of a tower, for instance, would appear to meet in a point if the tower were high enough, he thought that these sides ought to slope to one another in the picture. On this theory he published a small work (of which I have not the title) with a Grecian temple in the frontispiece; stated, if I remember rightly, to be the first picture which had ever been drawn in true perspective. Of course, the building looked very Egyptian with its. sloping sides. The answer to his notion is easy enough. What is called the picture is not the picture from which the mind takes its perception; that picture is on the retina. The intermediate picture, as it may be called, the human artist's work, is itself seen perspectively. If the tower were so high that the sides, though parallel, appeared to meet in a point, the picture must also be so high that the picture sides, though parallel, would appear to meet in a point 
I never saw this answer given, though I have seen and heard the remarks of artists on Mr. Parsey's work. ... In June, 1831, reading an article on squaring the circle, and finding that there was a difficulty, he set to work, got a light, denied to all the mathematicians, in - some would say through-a crack, and advertised in the Times that he had done the trick. Perspective has not had many paradoxes. The only other one I remember is that of a writer on perspective, whose name I forget, and whose four pages I do not possess. $\mathrm{He}$ denies that the stereographic projection is a case of perspective, the reason being that the whole hemisphere makes too large a picture for the eye conveniently to grasp at once-that is to say, there is no perspective because there is too much perspective.'

De Morgan includes among the paradoxes Bacon's theory that knowledge of Nature was to be obtained by 'the collection of enormous masses of facts, and then digested processes of arrangement and elimination, so artistically contrived that a man of common intelligence, without any unusual sagacity, should be able to announce the truth sought for.' Bacon's theory is undoubtedly opposed by all experience. His own description of his 'way of discovering sciences,' as one that 'goes far to level men's wits and leaves but little to individual excellence, because it performs everything by the surest rules and demonstrations,' reads as an absurdity in the presence of the history of the last two centuries. 'What are large collections of facts for?' says De Morgan. " "To make theories from," says 
Bacon. To try ready-made theories by, says the history of discovery. It's all the same, says the idolater. Nonsense, say we !' It is almost useless, however, to attempt to reason with the idolaters of the Baconian system; for it is a system pleasing to the men of little brains for whom Bacon promised so much. So observations are still being heaped together, too often without plan or purpose ; though, instead of the promised discoveries, we get blunders only as the fruit of all such labours. It still remains, and is likely long to remain, a cause of reproach if any man would stop the workers (save the mark!) to ask what they are working for; and scarcely any recognise the fact that science progresses despite these wasted labours, not in consequence of them.

It will be judged from this instance, that De Morgan does not reserve all his forces for the ordinary paradoxist, the circle-squarers, 'number of the beast' men, earth-flatteners, and so on. No inconsiderable portion of the work is directly given to the consideration of really important subjects, and indirectly, as I have said, it is full of interesting and valuable matter.

English Mechanic, 1872. 
THERE are reasons for believing that a thoroughly hearty blunder has a better chance of thriving than an average truth. Men still believe in the influence of the moon on the weather, and many even still suppose that the moon shifts her horns to announce weather changes, and not according to strict unchanging law; whereas only a few among the discoveries of science have ever found general acceptance. Nay, I believe to this day, if the civilised world were polled on the question of the earth's rotundity, and then on the question whether the moon rules weather changes, more would be found to vote in favour of the easily understood blunder than of the less readily understood truth.

As an instance of the vitality of attractive errors may be specially noted the recent revival of Dr. Stark's theory that marriage conduces to longevity. I take some interest in the vitality of this special error, as I chanced to be one of the first to point out the fallacious nature of the reasoning on which it was based. My paper, written anonymously in 1868 , was subsequently quoted by Dr. Farr, who had dealt with the influence of marriage on mortality in France in a paper read nearly 20 years ago before the Association for Promoting Social Science, and who is justly described by 
Darwin as our highest authority on such subjects. Darwin himself and Herbert Spencer have further indicated how the matter really stands. Yet the error has been repeated again and again since, and only a few days ago a learned professor, lecturing at the Montefiore Institute, quoted the fallacious evidence from statistics which deceived Stark, Bertillon, and others. Dr. Drysdale, indeed, goes somewhat farther. Not only, according to him, does matrimony prolong life, but it saves men also from insanity and from crime.

I have quoted Dr. Stark's reasoning in the essay above referred to, since published in the first series of my Light Science. It is based on the mortality returns in Scotland. Bertillon's later evidence, based on the mortality in France, Belgium, and Holland, is to the same effect, and runs as follows:- 'From 25 to 30 years of age the mortality per 1,000 in France amounts to 6.2 in married men, 10.2 in bachelors, and $21 \cdot 8$ in widows. In Brussels, the mortality of married women is 9 per 1,000 , girls the same, and widows as high as $16 \cdot 9$. In Belgium, the mortality of 7 per 1,000 among married men rises to $8 \cdot 5$ in bachelors and 24.6 in widowers. The proportion is the same in Holland. From 8.2 in married men it rises to 11.7 in bachelors and 16.9 in widowers; 12.8 in married women, 8.5 in spinsters, and 13.8 in widows. The result of all the calculations is that from 25 to 30 years of age the mortality is 4 in married men, 10.4 in bachelors, and 22 in widowers. This beneficial effect of marriage is 
manifested at all ages, being always more strongly marked in men than in women.' The superior mortality among widows and widowers can manifestly be explained otherwise than by assuming marriage to be beneficial ; if it could not, it might act to some degree as a deterrent, seeing that the possibility of becoming a widower or widow is one of the contingencies to which each man or woman who marries is exposed. But at a first view the general theory that marriage conduces to longevity seems to be made out by such statements as the above. So also Dr. Drysdale's statements are regarded, by those unfamiliar with the principles on which the interpretation of statistical evidence depends, as demonstrating, beyond possibility of doubt or question, the influence of marriage in preventing crime, insanity, and early death. It appears from French statistics (chiefly) that married men are less frequently charged with crime than bachelors, and that single women are much more criminal than married women. Among those who have to be sent to maisons de santé, again, are many more single men and women than married folk. 'Men are far more benefited by marriage than women, according to statistics,' proceeds the report of Dr. Drysdale's lecture ; 'but when men marry before twenty-three years of age, they die rapidly. With this exception there is a higher rate of mortality among bachelors than among married men. As to women, the married state is favourable to them after the age of twenty-five, and after reaching forty-five they live very much longer 
than if single. Young married women, like young married men, die rapidly.' As to men, all those from twenty-three to seventy who remain unmarried 'are practically committing suicide.'

It might be shown in the same way that matrimony is a preventive against poverty, bad looks, deformity, ill-temper, intemperance, and a number of other undesirable qualities, if statistics on these points were carefully collected; for, beyond all doubt, there would be found to be more poor, ill-looking, deformed, sourtempered, and drunken persons among the single than among the married. Or it might be shown quite as satisfactorily or unsatisfactorily (but with far greater probability of correctness) that to be poor, or ugly, or ill-tempered, or intemperate, conduces to singleness (' single cursedness' let us say). And the statistics actually quoted by Stark, Bertillon, and Drysdale may be used (as justly as by these statisticians) to show that circumstances which are unfavourable to longevity diminish the chance of securing a partner for life, and that tendencies to insanity or to crime are not, strictly speaking, advantageous features in either male or female candidates for matrimony.

The truth is that neither conclusion can justly be derived from the statistics quoted, though certainly one or other is just in itself, and most probably both are just. If more bachelors die than married men between given ages, it is certain that either the married state is favourable to longevity, or that persons likely to die soon, whether from weak health, intem- 
perance, poverty, or whatever cause, have not (on the average) so good a chance of marrying as others. And few who consider the facts carefully can doubt that both these inferences are sound. But the statistics cannot show whether one or the other is the true *explanation; or, if both causes operate, still the statistics do not show in what degree each is effective. The mistake made by Stark, Bertillon, Drysdale, and others, is in assigning the observed relation to one cause, or rather in saying this is cause and that is effect, when in reality cause and effect may, for aught the statistics show, be interchanged. It is, for instance, at least as probable that a tendency to commit crime diminishes the likelihood of a man's marrying as that his marrying diminishes the likelihood of his committing crime. I do not say that the former explanation of the asserted infrequency of crime among married folk is necessarily the true one. To assert this would be to make a mistake of precisely the same kind as that which mars. Dr. Drysdale's reasoning. At least, if the assertion were based solely on the statistics, for these can prove nothing of the sort. On à priori grounds the assertion has far more in its favour than the other, for it is difficult to conceive any reason why matrimony should diminish criminal tendencies to any marked degree (though a man's marrying might be evidence that he had been tamed); whereas one can see many reasons for believing that a man with criminal tendencies would be regarded as an undesirable suitor. If the argument of Dr. Drysdale, though demonstrably 
unsound, tended primarily to encourage matrimony, and still more, if it tended to discourage immorality, I should not care to indicate its unsoundness; though I have always maintained that the student of science should set truth above all other considerations. But I take it that of all possible resources for those of tainted or feeble constitution, of strongly criminal tastes, or of insane tendencies, matrimony is, on the whole, the most objectionable, so far as the community at large is concerned. If a consumptive person could add ten years to his life by marrying, he might also add ten consumptive persons to the next generation, and who shall say how widely the taint would spread in later years. The criminal is hardly likely to be influenced by other than selfish considerations ; but most certainly the community has no reason to desire that he should be encouraged to increase and multiply criminality. As for a person who inherits a tendency to insanity, and knows it, such a one, if he entertains while still sane the thought of marrying, is a criminal of deepest dye, and the worst possible thing that can happen to the community is that his insanity should delay to manifest itself until the taint has spread to his innocent offspring.

But the scientific objection to Dr. Drysdale's reasoning is that it is statistically unsound. 


\section{INCREASE OF THE POPULATION.}

In the discussions which have taken place upon the question of population, and especially upon the views of Malthus, two points have very generally been confounded which Malthus himself was very careful to distinguish. The theory of population as it relates to the community is one thing; the course of individual members of the community is another. But in recent discussions the arguments which bear, or are supposed to bear, on the theory of population, have been used as though they were the arguments which should guide the course of individuals. Is it really supposed by political economists and statisticians that a labourer (for example, though the same reason might be applied to every class of the community) is likely to care two straws about the matters which they have submitted for his consideration? In considering the probable or possible addition of a new member to his family, does he examine the relation between increase of population throughout the country and the average rate of food increase? Does he inquire how far it is desirable for the country at large that the birth-rate should be maintained at such and such a value? Does he consider that our power as a colonising nation will suffer, or our armies be unduly reduced, if the average number of children per family falls short of its present value? He may, for aught that is known to the 
contrary, consider vaguely the possibility that among the coming members of his family there may be a Shakespeare or a Newton. Dickens, a close observer, pictures Mr. Kenwigs, who may be regarded as fairly typifying the British paterfamilias of a certain class, propounding to himself the momentous query, 'If it's a boy, as I hope it may be, . . . will he be Alexander, or Pompey, or Diorgeenes, or what will he be?' But the general argument that men should increase and multiply in order that Shakespeares and Newtons may have a fair chance of coming into the world does not, probably, weigh very much with the average Briton. It is not, indeed, altogether clear that even as a general argument it has any specific value; because assuredly the chance of bringing into the world some notorious malefactor is greater on the whole than that of producing a Goethe or a Shakespeare, a Laplace or a Newtonseeing that great malefactors have appeared in considerably larger numbers than great poets or great philosophers.

The only consideration likely to have any influence with individual members of the community, or therefore to influence the result at all - for the result depends entirely on the action of individuals-are those which appeal either to the sense of personal duty, or to the sense of personal interests. All else that has lately been said or written may be exceedingly interesting, just as it is exceedingly interesting to inquire into the effects of gravitation or any other law of nature; but can have very little practical value, simply because it 
has no practical application. Consider, for instance, Malthus's reasoning in favour of late marriages, and the evidence which has been adduced from the experience of different countries where folk marry either exceptionally early or exceptionally late. Has any couple, since Malthus propounded his views, either deferred their marriage because they accepted his reasoning, or hastened their marriage because they rejected his conclusions? Imagine a young lover urging the lady not yet to name the day (or the lady declining to do so, for the population question belongs equally to both sexes), because ' it has been shown by Mr. Malthus, in his Essay on Population, second edition, 1803, that among all the preventive and positive checks upon the rapid increase of population, the prudential restraint from marriage, with conduct strictly moral during the period of such restraint, is the most desirable, and, in fact, the only proper one!' The actual rate of increase of population, again, under ordinary conditions, and the relation of this increase to the means of subsistence in countries densely, moderately, and thinly peopled, are matters full of interest in themselves, but not having the least bearing on the conduct of individual members of the community. If the bulk of the population were neither able, to regulate their own conduct, nor (which is another matter, by the way) in the habit of so doing, if they at every stage of life waited always to hear what the British Association or the Social Science Congress had

III. 
to say (in matters over which the powers that be do not assume control), the case would be different. It might be worth while, in that case, to argue about the population question in its national and social aspect. But, in point of fact, our people marry and are given in marriage according to their own ideas of what is right or desirable; and as to the question of family fertility, the only way in which argument can possibly affect this question is by making the generality acquainted with facts which had not before been widely known, however familiar to physiologists, and through them to all classes of nature students. In other words, argument can act in one direction only in this matter. Not one person throughout the length and breadth of the British Empire will be led by any of the arguments which have been so profusely urged to modify his ideas, either as to the desirability of a numerous progeny, or as to the direction in which duty points; but many may first learn through these discussions that the question of duty enters into the matter at all (for what is regarded as, to all intents and purposes, inevitable, cannot be considered either a duty in one aspect, or an offence in another).

On another point I would indicate a circumstance in which, I think, the tone of these discussions might be advantageously modified. Whether the discussions avail or not to modify the conduct of individuals, and thus affect the wellbeing of the community-either favourably or the reverse-it is certain that nearly everyone who takes part in them on either side does 
so from good motives. On the one hand, there are many who attribute a large proportion not only of the misery, but of the immorality, actually existent among us to overcrowding, not in the wider sense in which countries and districts are overcrowded, but as overcrowding affects the houses or homes of individuals. On the other hand, there are many who consider not only that increase of misery is amply compensated by the existence of a new soul, and the chance which this soul has of future beatitude (to say nothing of the necessity which, it would seem, exists for new recruits both above and below), but that, whether there is compensation in this way or not, men have no choice in the matter: their bounden duty is to increase and multiply as fast as Nature will permit. This being so, it would seem that these good motives on both sides should be fairly recognised, and charges of impiety on the one hand, and of want of feeling on the other, should be avoided. It would be impious, doubtless, for those who regard increase and multiplication as divine commands to advocate any limitations, whether by late marriages or otherwise, to the development of our population; and, on the other hand, it would be decidedly wrong of those who consider, with Mill, that ' causing the existence of a human being is one of the most responsible actions in the range of human life,' to urge that men should undertake this responsibility where the life bestowed is far more likely to be a curse than a blessing - a curse not only to the creature called 
into being, but to those whose misery, already scarce endurable, that creature will share and increase. But, seeing that on each side men urge arguments which seem to them just, neither side deserves to be accused of wrong-doing, whether in the way of impiety or of selfish disregard to the misery of their fellows. This would be true even if the arguments on either side really influenced the conduct of others. Seeing that the whole discussion (except in so far as it spreads among the many information which had been limited to the few) can only point to facts real or surmised, but can in no sensible degree affect conduct, it really does seem that acrimony might well be avoided on both sides.

As to the plans of the Creator in this matter, which some writers have been careful to defend, I would submit that we know little about them, and, even if we knew a great deal, those plans would hardly need defence. It would be, perhaps, rather difficult and a trifle dangerous to define the original divine plan of population. The most orthodox must admit that human intervention has to be taken into account, whether as involved in the divine plan or not is a matter of no moment, for in either case we come round to the question how such intervention is to operate. Although three children are born for each that grows to maturity, it has not yet been demonstrated that either early marriages or large families are essential parts of the divine plan for peopling this world and the next. 
Probably individual ideas of duty, propriety, and self-interest will continue to govern men's actions, as heretofore, though it will be found, I think, before many years have passed, that the facts published during recent discussions have been somewhat widely noted.

Echo, 1878.

COLLISIONS AT SEA DURING FOG.

THE care with which money is saved over the signallamps of ships, \&c.--especially steamships at nightis most praiseworthy. Several pounds a year must be saved by using only two side lights - the red port light and the green light on the starboard side ; and what if, when a collision occurs, half-a-million of money's worth may go in a few minutes to the bottom ? A ship lost is a ship lost, but economy must be attended to as allimportant.

But seriously, when we consider the power which science has attained over the forces of nature, when we remember how, by virtue of such power, we can make our ships independent of wind and tide, and how we urge them-each with its freight of living soulsalong the great trade routes in hundreds, it does seem. to imply stupidity and want of care, to say nothing worse, that such means as science affords for defending them against the risk of collision should not be employed. I will not here consider the general question 
of collisions at sea, though it is one about which much might be said. But such disastrous collisions as have occurred recently might easily be avoided, or at least rendered extremely infrequent, if proper means were used for indicating, by strong lights suitably placed, the position of steamships, whether in motion or at rest. Everyone who has traversed a thick fog, even a London fog, since the electric light has been much used in towns, knows how much farther a strong electric light shows in a fog than the best gas-light, and (still more) than any oil-light. It is well-known, too, how much the use of any coloured glass diminishes the range of distance through which a light can be seen. Now, it may very well be that the general use of the electric light on board steamships may not be possible at present. I should have thought the owners of our best ocean steamships would long since have decided to use only the electric light for their signallamps, if not for illuminating purposes throughout those ships. But supposing this not yet possible or convenient, it is yet obvious that in every steamship, either slowed or brought to rest during fog, there is available a store of energy which might be well employed at those critical times to drive a dynamo machine and maintain any desired number of very strong electric lights in suitable positions. Such lights would penetrate to a considerable distance through any ordinary sea fog. If they had been burning on the 'City of Brussels' and the 'Kirby Hall,' or on the 'Cimbria' and 'Sultan,' it is, to all intents and purposes, certain 
that the late disastrous collisions would not have occurred. The mere indication of the presence or approach of these several ships would have sufficed to have averted the danger.

But more than this might very readily be done. The lights at present used are of very little service in indicating the position in which a ship lies. A red light shows that her port side would be in view were it day, but not how that side is presented. It may be the broadside which is towards another vessel, or she may be lying with her bows only so far turned to starboard from being directly end on that the slightest possible change in her course or bearing will bring the green starboard light into view. (Precisely such a change happened just before the 'Cimbria' was run down.) Now there is absolutely no reason whatever (save the expense required for some eight or nine instead of two or three lamps ${ }^{1}$ ) why this uncertainty should exist. Supposing only side lights can be used, instead of such central lights as might, perhaps, be hidden by sails or rigging, then what is to prevent such an arrangement as the following from being used along the port and starboard sides of every shipespecially of every steamship?

1 It may be objected that the use of three lights instead of one light on each side would be confusing; but matters might very readily be so arranged that the three side lights would practically be regarded as one. Indeed, they ought to be so close together that, at the distance at which a ship's side lights are usually seen, they would appear as a single light of triple strength; a little nearer they would show a triangular form; and, when within the distance at which there is anJ danger of collision, they would be separately visible. 


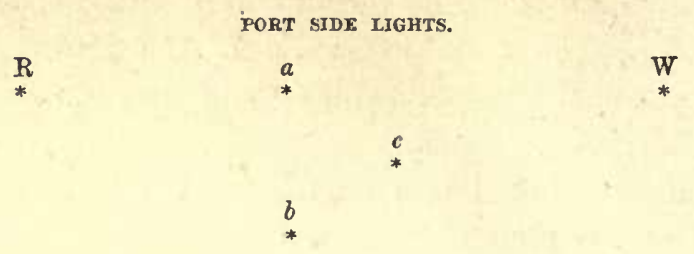

STARBOARD SIDE LIGHTS.

W

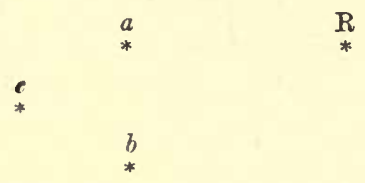

(W a powerful white light at the bow, $\mathrm{R}$ a powerful coloured light at the stern, $W$ only visible when the bow is nearer than the stern to observer, $\mathbf{R}$ only visible when the stern is nearer to observer than the bow; $a b c$ the midship port lights arranged in the form of an equilateral triangle.

A little consideration will show that, if such lights were used, not only would the port side be distinguished as now and from the starboard one much more quickly, because white lights are seen much farther than coloured ones, but the exact bearing of the ship's midships would be at once shown by the foreshortened shape of the triangle $a b c$.

Newcastle Weekly Chronicle. 


\section{THE EYES OF SCIENCE.}

THE telescope, the microscope, and the spectroscope give to the student of science what may be regarded as three kinds of visual power-in one case unlike the visual qualities possessed by the natural eye, in the other two surpassing these greatly in degree. We can conceive creatures endowed with the powers of vision which the telescope and the microscope artificially supply. Indeed, it is by no means incredible that in other worlds than ours creatures may exist possessing powers akin to these. And although it is not easy to conceive the sense of vision so increased and extended that by means of it the analysis of light effected artificially by the spectroscope could be effected naturally, yet there is nothing absolutely outside the range of possibility, even in this. The eye is, indeed, an optical instrument, precisely as the ear is an acoustical instrument; and so far as we can judge, the sense of vision might have been provided with a more complex organ, or series of organs, giving it greater range, as in the telescope, or more complete power of magnifying minute details, as in the microscope, or the power of separating light rays of different refractive nature, as in the spectroscope. There are other optical instruments also whose powers might have had their analogues in the organ of sight (as the polariscope and similar instruments); while there are others, as the stereoscope 
and so forth, which, like the telescope and microscope, are akin to the organ of vision, but give to it increased power in particular ways.

I have lately been led to notice how certain photographic processes and methods extend the powers of human vision, and enable us to see what, owing to certain peculiarities in the circumstances under which eyesight is employed, we are debarred from seeing in the ordinary way.

It has long been noticed that photographic vision, so to describe this method of studying natural objects, has one great advantage over ordinary vision in that it is not liable to ordinary misleading influences. In science, seeing is not always, or even generally, believing-for the simple reason that the student of science cannot always be certain what he really sees.

Thus an observer may be misled by imagination, especially if some favourite theory has possession of his mind. If he knows, or thinks he knows, what he ought to see, or might fairly expect to see, he is very apt to imagine that he actually does see it. In this way, for instance, many students of astronomy have fancied they have seen a small companion by a star in a position where they had been told such a companion existed, when, in reality, there had been some error in the description, or in their reading of it, and either no such companion existed, or else it was in some entirely different position, and perhaps quite beyond the range of the telescope employed by the observer.

Again, the eye is repeatedly deceived by effects of 
contrast. Thus, the French astronomer Chacornac advanced a very ingenious-indeed, masterly-theory in explanation of the circumstance that the disc of the planet Jupiter is brighter near the edge than in the middle, the only objection to his theory residing in the circumstance that the disc is darker, not brighter, near the edge, though to the eye it appears brighter there by contrast with the dark background of the sky on which it is seen projected. So again there is a charming theory, in vogue to this day among many students of the moon, explaining why the floor of the lunar crater Plato (the Greater Black Lake of the earlier telescopists) grows darker as the sun pours more light upon it (rising higher in the sky as supposed to be viewed from Plato), the real fact being that there is no such darkening, the apparent difference being entirely due to effects of contrast-the contrast of the floor with the black shadows of the crater-ring thrown upon it when the sun is low, and the contrast of the floor with the brilliant white of the surrounding craterring when the sun is high, one contrast making the floor look lighter than it is, while the other makes it look darker. I may cite another instance of an optical illusion, caused by an effect of contrast - a case not requiring telescopic observation for its recognition. If on a moonlit night one looks beyond a water horizon towards the part of the sky below the moon, that region looks darker than the parts of the sky on either side; yet, in reality, it is no darker-if anything slightly lighter. What causes it to look darker is the 
apparent brightness of the part of the water just below the moon, where lies seemingly ${ }^{1}$ a broad track of silver light. If this track of light is concealed in any way, as by holding up a sheet of card or paper, the portion of the sky immediately above is at once seen to be at least as bright as the parts of the sky on either side of it. So in multitudes of other cases, some familiar, some otherwise-the eye is deluded by effects of contrast.

Photography, or what may be called photographic vision, is not, it is true, altogether free from defects corresponding to such defects of vision (resulting in illusion) as we have just considered. As there are physiological illusions in ordinary vision in such cases, so are there in certain applications of photography, physical effects which may prove similarly illusive. For instance, there is what is sometimes called photographic irradiation, when around a dark object in a photograph a ring of light is seen, or around a bright object a ring of darkness, this ring not corresponding to any really existent object, but resulting from some change in the photographic film along the border-line around a region acted on very strongly by light.

Again, the photographic eye has long been justly valued for its artistic power, in being able to record, without defect or exaggeration, what it sees. If we

'I say ' apparent' and 'seemingly ' because the moon's rays really illuminate the region which appears dark, as brightly as the rest. It is only because of the position of the observer's eye that one region appears brighter than the rest. 
take, for instance, one of Dr. Rutherford's photographs of the solar disc, and compare the spots there depicted with those shown even in the most carefully executed pictures of the sun before and since, we see at once how liable the eye is either to be deceived in what it sees, or else to fail duly to guide the hand in reproducing what the eye has seen. I happen to know of a case where a draughtsman took exceptional pains to reproduce, without exaggeration, the aspect of the solar disc with its spots, yet, on comparison being made with a photograph taken nearly at the same time (though 3,000 miles away), it appeared that the spots had been notably exaggerated. I refer to the drawing of the sun's face which forms the frontispiece of my treatise on The Sun. I was particularly struck by the aspect of the sun when that drawing was made, and I certainly spared no pains to delineate the spots correctly; but a comparison of my picture with a wellknown photograph by Rutherford, which chanced to be taken about the same time in New York, will show that though the spots are delineated, individually, correctly enough, they are considerably too large as compared with the solar disc-an enlargement by no means necessary to enhance their importance, for the largest spot visible on that occasion had a surface several times larger than the entire surface of this earth.

Other instances of the same sort may readily be cited. A very noteworthy case, in which the imperfection of ordinary vision and ordinary methods of 
delineation caused many men of science to be long led astray, till photography finally came to their rescue, is that of the solar corona. The pictures of the corona which used to be drawn by different observers of the same eclipse, often by observers stationed within a few yards of each other, showed such diserepancies as to suggest to some the theory that the corona is not really a solar appendage, but an optical phenomenon, caused by the passage of the sun's rays through our own atmosphere; and although a very slight knowledge of mathematics sufficed when applied (for many mathematicians, failing to apply their knowledge, were long misled) to show the erroneous nature of this theory, it was not until photography had been employed to delineate the corona, that the groundlings were convinced on this comparatively simple point. A singular illustration of the inferiority of the unaided vision in this matter was given once at a meeting of the Astronomical Society. 'Two people,' said Mr. Stone, referring to the eclipse of 1875 , which he had witnessed in South Africa, ' were asked to make drawings of the corona, and at the end of the time one man had drawn it in one shape, and the other as different as it could possibly be.' Considerable amusement was caused by Mr. Stone's sketching two absurdly dissimilar pictures on the blackboard. The person who drew one picture was an engineer, and was sitting side by side with the other. Before the eclipse was over he turned round to look at the other's drawing, and said, 'What on earth are you doing here?' He replied, 'I am drawing the 
outer corona.' He said it was nothing of the kind; yet he looked and saw the outer corona just as the other had drawn it; but he had overlooked that it was the inner corona he was drawing, until his attention was called to it in that way.

On the same occasion Mr. Stone pointed out another defect of ordinary vision, as an instrument of scientific research, which is well worth noticing. He said very truly, that as soon as any feature has arrested the attention, it becomes prominent; another person may not catch the same feature, and, passing it over, seizes upon some other point and brings it out more strongly. Photography has no weakness of this kind, at least where due care is taken to use the same kind of plates and instruments, nearly equal in size and similar in quality, when comparison is to be made between pictures taken by different observers or at different stations.

Knowledge, June 23, 1882.

GREAT SUN SPOTS.

Whatever success science may have, or fail to have, in predicting terrestrial weather, there can be no doubt astronomers have learnt to predict with considerable correctness the occurrence of the mighty solar storms which produce what are called sun spots. They cannot yet say that on such and such a day, or 


\section{LIGHT SCIENCE FOR LEISURE HOURS.}

even in such and such a week or month, a great spot will appear; but they can tell what years will be characterised by many sun storms and what years by few, for ten or twelve years in advance. The great sun spots which have been seen during the last few months were predicted at least twelve years ago ; and astronomy is far better assured that in the years 1893 and 1894 there will be many large sun spots than meteorologists are that next March will probably be stormy and next June relatively calm. Yet scarce half a century has passed since the periodicity of sun spots began to be recognised, and not a quarter of a century has passed since the theory was thoroughly established. We do not even yet know why these waves of sun spots pass in their long ten-yearly surge over the vast surface of the sun. The Kepler of the sun has done his work; the Newton has yet to come. The work of a solar Newton will be well worth doing, even though he may not (as he probably will) bear somewhat the same relation to Schwabe that the profound Newton bears to the ingenious and laborious Kepler.

What a problem it is that lies before astronomers when we consider what sun spots really mean! The great atmosphere of the sun, whose breath is flame, is yet so cool compared with his intensely glowing surface that it absorbs a large proportion of his light as well as of his heat. It absorbs so much that it actually changes his colour. There can be no manner of doubt, from what Professor Langley has shown about the 
absorptive qualities of that atmosphere, that were it suddenly stripped off, the sun would shine not only with greatly increased brightness, but with a bluish violet colour. In a very short time indeed that colour would seem white again to our eyes, grown accustomed to the change; after which, the sudden restoration of the absorbing atmosphere would change the sun to an orange-red orb, which only after awhile would seem to our eyes a white globe as before. But while the general absorptive action of the sun is wonderful, the story is still more wonderful which the spectroscope has to tell about the specific absorptive effects due to its constitution. We find that, whereas in our air the vapour of water is present (to condense into water drops and form clouds at certain levels, and to change to ice-crystals and form cirrus at higher levels), in the sun the atmosphere is laden with the vapours of iron, copper, zinc, sodium, magnesium, and like elements, to form clouds of metallic drops, great gatherings of metallic crystals, while the rains that pour down towards the concealed true globe of the sun are mighty showers of molten metal. When a hurricane occurs in the sun, the clouds which form the sun's surface are swept along, or whirled around, not at the rate at which we measure our storms, but with a velocity compared with which their swiftest motion is as rest. The solar tornadoes rage, not over a few hundred square miles, but over regions as large as the whole surface of the earth, over hundreds, even thousands of millions of square miles; and they travel over these enormous III. $\mathrm{x}$ 
regions at a rate not of so many miles per hour or per minute, but of many miles, sometimes more than a hundred miles, in every second of time. Such storms are in progress now, where we see the spots upon the sun. Such storms tell us of the activity of that great central engine whose throbs are the life-beats of the solar system.

We measure the sun's work, perforce, by our own forms of work. We speak of his emission of light and heat as corresponding to what would result from the burming of eleven thousand millions of millions of tons of the finest coal in every second of time. But what mind can conceive the real vitality of that mighty orb which seems so silent and so still in our skies? The throbbing of the great engine which beats out light and life to the whole family of planets can only be seen by the mind's eye, and as yet that eye is no more capable of seeing the sun's work as it really is than is the bodily eye of seeing the distant millions of suns which the great gauging telescopes of the Herschels bring within our ken. Nor can the mental ear hearken to the uproar and tumult with which the work of the great central engine is accomplished, or imagine what would be heard if one could visit that spot which looks like a tiny speck on the sun's surface, and, passing below the limits of the solar air so that sound waves could reach him, could find (as assuredly he would if he could live at a temperature which turns the hardest metal into vapour) all forms of noise known to us-the roar of the typhoon, the crash of thunder, 
even the hideous groaning of the earth-throe-surpassed a millionfold by what takes place within every square mile of that disturbed region.

One cannot wonder if many students of science are eager to find out the real meaning of the sun spots, to learn how they are generated, and to solve the secret of that strange law which brings them in undulations ten or twelve years long over the surface of the sun. Still less can one wonder if many should be attracted by theories associating terrestrial phenomena, not in general (as they must assuredly be associated) but in detail, with the periodicity of solar disturbance. It has been shown that the earth as a whole responds to the solar action displayed in sun spots. There can scarcely be any doubt that the connection long since indicated by Sabine between the phenomena of terrestrial magnetism and the condition of the sun's surface with respect to spots is a real one. Not magnetic relations simply, but others which have only been associated within recent times with magnetism, as the occurrence of auroral displays, \&c., have been clearly associated with the general condition of the sun's surface on the one hand, and with the outbreak of specific sun spots on the other. True, the great solar storms recognised, when mighty masses of glowing gas have been flung forth in the form of prominences, have occurred without any simultaneous auroral or magnetic disturbances on the earth. But these are side issues, literally. The solar energy is there directed not towards the earth, but at a right angle or there- 
abouts with her direction; and we can hardly wonder if she does not respond to these solar asides. Whenever the face of the sun turned towards her has shown evidence of perturbation she has responded quickly enough. The disturbance of September, 1859, was answered by movements of the magnetic needle at Kew, which, if not actually simultaneous, were so nearly so that the light of the sun itself reached us no quicker than the influence exciting that magnetic disturbance. Nor did the tremulous response of the perturbed earth last but for a moment. Throughout the night that followed Arctic and Antarctic auroral banners waved over the northern and southern hemispheres, being visible in latitudes seldom reached by such displays. For more than twenty-four hours, also, telegraphic communication was interrupted.

Again, it seems clear that the temperature of the earth, as a whole, is affected by the absence or presence of many spots on the sun's surface. This has been shown apparently in an unmistakable way, by the underground thermometers at Edinburgh and at Greenwich. But the rain and wind cycles, the famine and financial crisis periods, the recurrence of disasters and shipwrecks, bad vine years, and so forth, in harmony with the sun spot waves-these have not yet been established. It sounds convincing when one cyclist notes that over a certain region the north-east winds are wetter and the south-west winds drier in sunspotted times than when the sun is free from spots. Others find it still more convincing when some one 
else finds that in another region the reverse holds. And when it is further found that in some regions no such effects at all can be discerned, many find nothing disheartening in that. Still, it must be remembered that antecedently this sort of evidence was certain to be obtained whatever period had been dealt with; looking over a short range of time, one would be sure to find some places where the weather seemed to agree in one way with the period (any period whatever), other places where the weather seemed to agree in just the opposite way, and yet others where there seemed to be no agreement at all. And when we learn that as our survey ranges over time as well as over space, there appear similar diversities, the places which had seemed to agree one way or another no longer agreeing, it seems a little too much to ask men to believe that there is a real connection, but that while one place is affected one way, another is affected in the opposite way, and that as time passes, the effects vary. With such scope for difference and variation, a pack of cards, shuffled at random, might be shown to agree with weather cycles (red cards for fine weather, black ones for bad, or vice versâ ad libitum). Weather predictions guided by sun spots would be no better, in that case, than predictions based on coin-tossing suggested by Sir Edmund Beckett.

Times. 


\section{WORKS BY THE SAME AUTHOR.}

LESSONS in ELEMENTARY ASTRONOMY; with an Appendix containing Hints for young Telescopists. Third Edition, enlarged, with 47 Woodcuts. Fep. 8vo. price 18. 6d.

ELEMENTARY PHYSICAL GEOGRAPHY. With 33 Maps, Woodcuts, and Diagrams. Fcp. 8vo. price 1s. 6d.

The ORBS AROUND US; a Series of Familiar Essays on the Moon and Planets, Meteors and Comets, the Sun and Coloured Pairs of Stars. Second Edition, with Chart and 4 Diagrams. Crown 8vo. 7s. 6d.

The MOON ; Her Motions, Aspect, Scenery, and Physical Condition. Revised Edition, with 22 Lithographic Plates, Charts and Diagrams, 1 Wood Engraving, and 3 Lunar Photographs. Crown 8vo. price 10s, 6d.

The SUN ; RULER, LIGHT, FIRE, and LIFE of the PLANETARY SYSTEM. Revised Edition, with 10 Plates (7 Coloured) and 106 Figures engraved on Wood. Crown 8ro. price 148.

- The fact that such a book as the presents should have reached a third edition says much for the growing taste for works of an educational character among the reading public. It is perhaps the most interesting of the series of publications, astronomical and scientific, issued by $\mathrm{Mr}$. Proctor. The new edition appears to have been carefully revised, and some important sections have been added to it. The appendix on Transits of Venus has wisely been removed, the purpose for which the essay was written having been accomplished. The reader has placed before him all that is known, or what may be surmised, of the great ruling luminary of our system; the dis-

coveries that have been effected by means of the telescope, the spectroscope, polariscopic analysis, and photography. There are treated very fully the questions of the sun's distance, his influence as ruler over the system of planets, his physical condition, and his place and motions among his fellow suns; while reasons are presented for considering that the most important work science has to accomplish is to show how the sun's action can be more fully ntilised than it is at present. We should not omit to state that the book is enriched with nine lithographic plates (seven coloured) and one hunired drawings on wood.'

The LANCET.

ESSAYS on ASTRONOMY; a Serics of Papers on Planets and Meteors, the Sun and Sun-surrounding Space, Stars and Star Cloudlets. Revised Edition, preceded by a Sketch of the Life and Work of Sir John Herschel. With 10 Plates and 24 Engravings on Wood. 8vo. price 12 s.

OTHER WORLDS THAN OURS; the Plurality of Worlds Studied under the Light of Recent Scientific Researches. With 14 Illustrations. Crown 8vo. price 108. $6 d$.

The UNIVERSE of STARS; presenting Researches into the Now Views respecting the Constitution of the Heavens, with an Investigation of the Conditions of the Transits of Venus. With 22 Lithographic Charts (4 Coloured) and 22 Diagrams on Wood. 8vo. price 10 s. $6 d$. 
A NEW STAR ATLAS, for the Library, the School, and the Observatory, in 12 Circular Maps (with 2 Index Plates). Intended as a Companion to 'Webb's Celestial Objects for Common Telescopes.' Revised Edition, with an Introduction on the Study of the Stars, illustrated by 9 Diagrams. Crown 8 vo. price 5s.

A STAR ATLAS for STUDENTS and OBSERVERS, showing 6,000 Stars and 1,500 Double Stars, Nebulæ, \&c. in 12 Maps on the Equidistant Projection; with Index Map on the Stereographic Projection. Fourth Edition. Folio, 158.

LIGHT SCIENCE for LEISURE HOURS; Familiar Essays on Scientific Subjects, Natural Phenomena, \&c. 3 vols. Crown 8vo. price 7s, 6d. each.

A TREATISE on the CYCLOID and on all Forms of Cycloidal Curves, and on the use of Cycloidal Curves in dealing with the Motions of Planets, Comets, \&c. and of Matter projected from the Sun. With 161 Diagrams. Crown 8vo.10s.6d.

TRANSITS of VENUS ; a Popular Account of Past and Coming Transits from the First Observed by Horrocks A.D. 1639 to the Transit of A.D. 2012. Fourth Edition, including an Account of the Successes Achiered in December 1874, and Snggestions respecting the Transit, December 1882. With 20 Plates (12 Coloured) and 38 Woodcuts. 8ro. 8s. $6 d$.

\section{STUDIES of VENUS-TRANSITS ; an Investigation of} the Circumstances of the Transits of Venus in 1874 and 1882. By R. A. Proctor, B.A. Cantab.' (Originally forming part of 'The Universe and the Coming Transits.') With 7 Diagrams engraved on Wood and 10 Plates of Figures. 8ro. 5 s.

\footnotetext{
- For those readers and students who wish to understand the exact nature of the questions to be decided by the forthcoming foreign expeditions to observe the Transit of Venus on Dec. 6 of the present year, Mr. Proctor's essays will form a valuable contribution to the comprehension of the case. His little book consists of several interesting studies, selected from the monthly notices of the Royal Astronomical Society or other like sources, all written with that perspicuous Incldity whlch enables the Author to put eren unmathematical readers immediately in possession of the right polnt of view. In fathoming these apparently difficult but really very simple conceptions, the student or reader will bo much aided
}

by the admirable and very careftul maps. Mr. PROCTOR, in fact, is a born mapmaker; be loves pntting things graphically on paper; and whatever be puts he puts 80 clearly that nobody with a little patience and application can possibly fail to understand bis meaning. Whllst most other astronomers think in terms of symbols and mathematical formule, it is Mr. Procton's specisl distinction that he seems to think in terms of concrete reality-to plcture the ohjects vividly before his mind's eye in their actual relations, instead of envisaging them under the guise of $x$, $y$, and $z$, worked out in sheet after sheet of algebraical calculation.'

Pall dall GazetTe. 



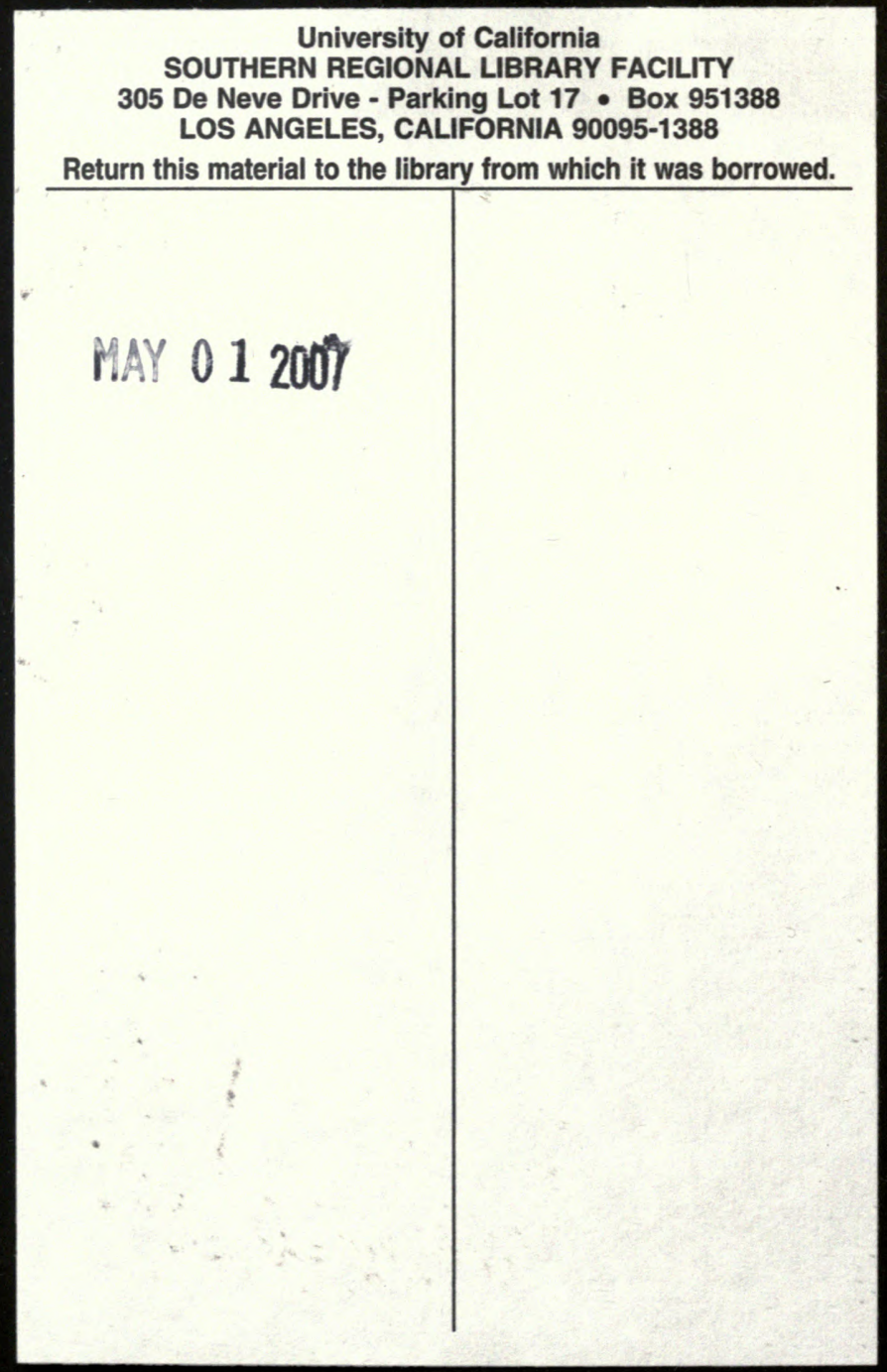



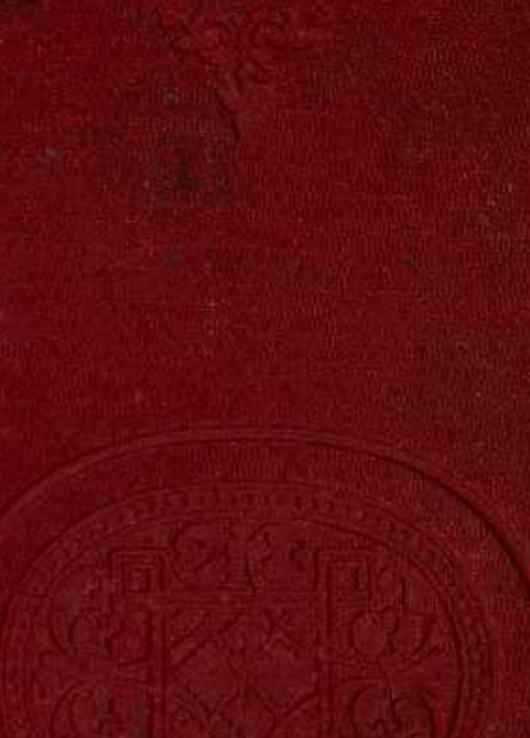

PW.

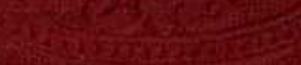

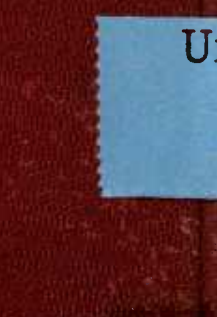

constion

40

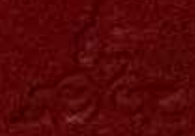

